\title{
Endogenous completeness of diffusion driven equilibrium markets*
}

\author{
Julien Hugonnier ${ }^{\dagger} \quad$ Semyon Malamud ${ }^{\ddagger} \quad$ Eugene Trubowitz ${ }^{\S}$
}

\begin{abstract}
We study the existence of equilibria with endogenously complete markets in a continuous-time, heterogenous agents economy driven by a multidimensional diffusion process. Our main results show that if prices are real analytic as functions of time and the state variables of the model then a sufficient condition for market completeness is that the volatility of dividends be nondegenerate. In contrast to previous research, our formulation does not require that securities pay terminal dividends and thus allows for both finite or infinite horizon economies. We illustrate our results by providing easily applicable conditions for market completeness in two benchmark cases: that where the state variables are given by a vector autoregressive process and that where they are given by a vector of autonomous diffusion processes. We also provide counterexamples which show that real analyticity cannot be dispensed with if one is to deduce dynamic market completeness from the structure of dividends.
\end{abstract}

Keywords: continuous-time finance, dynamic market completeness, general equilibrium theory.

JEL Classification. D51, D52, D53, G11, G12.

${ }^{*}$ First version: December 2008. This version: August 26, 2009. We wish to thank Tony Berrada, Peter Bossaerts, Rodolfo Prieto and Marcel Rindisbacher for conversations on the topic of this paper. Financial support by the Swiss Finance Institute and by the Swiss National Center of Competence in Research "Financial valuation and Risk management" (NCCR FinRisk, Projects A4 and D1) is gratefully acknowledged by Hugonnier and Malamud.

${ }^{\dagger}$ Ecole Polytechnique Fédérale de Lausanne and Swiss Finance Institute, Quartier UNIL Dorigny, Extranef, CH-1015 Lausanne, Switzerland. Email: Julien.Hugonnier@epfl.ch

${ }^{\ddagger}$ Ecole Polytechnique Fédérale de Lausanne and Swiss Finance Institute, Quartier UNIL Dorigny, Extranef, CH-1015 Lausanne, Switzerland. Email: semka@math.ethz.ch

${ }^{\S}$ ETH Zürich, Department of Mathematics, CH-8092 Zürich, Switzerland. Email: eugene.trubowitz@math.ethz.ch 


\section{Introduction}

Ever since the seminal contributions of Kreps (1982), Duffie and Huang (1985) and Duffie (1986) the standard way of constructing securities market equilibria in continuous-time economies with heterogenous agents has consisted of three steps. First, compute an Arrow-Debreu equilibrium. ${ }^{1}$ Second, define candidate prices for the traded risky securities by using the consumption price process of the Arrow-Debreu equilibrium as a pricing kernel and, third, verify that these prices give rise to dynamically complete markets.

The last step in the above program is crucial in establishing the existence of an equilibrium. Otherwise there is no way to guarantee that the consumption allocation of the Arrow-Debreu equilibrium can be implemented by dynamic trading in the given set of securities. Unfortunately, this last step is also the most technically difficult since the candidate prices are given by conditional expectations which cannot be computed explicitly in general. ${ }^{2}$ The only case where the verification of market completeness is straightforward is when there is a single state variable and a single stock in the economy. ${ }^{3}$ However, this assumption is far from being satisfactory as it does not allow for a study of the cross-sectional properties of equilibrium prices.

In representative agent economies endogenous market completeness does not matter for the existence of an equilibrium but it is nonetheless important for two reasons. First, the microeconomic justification for representative agent economies relies on aggregation results which require complete markets, see Constantinides (1982). Second, it is now quite common in the asset pricing literature to start from a representative agent economy and then use the equilibrium pricing kernel "outside the model" in order to price securities, such as derivatives, which were not included in the original menu of traded assets. ${ }^{4}$ Such a use of the equilibrium pricing kernel requires complete markets since only in that case does the derived price give the minimal amount necessary to replicate the given cash flows by trading in the set of primitive securities.

\footnotetext{
${ }^{1}$ Existence results for Arrow-Debreu equilibria in infinite dimensional economies can be found in Duffie and Zame (1989), Karatzas, Lehoczky, and Shreve (1990), Mas Colell and Zame (1991), Anderson and Raimondo (2008) and Section 2.5 below.

${ }^{2}$ Examples of equilibrium models where the prices can be computed in closed form include those of Santos and Veronesi (2006), Cochrane, Longstaff, and Santa Clara (2008), Hugonnier (2008) and Martin (2008).

${ }^{3}$ See Dumas (1989), Wang (1996), Chan and Kogan (2002) and Longstaff and Wang (2008) among others for examples of papers which consider heterogenous agent economies driven by a single state variable.

${ }^{4}$ Examples of papers which follow such an approach include Benzoni, Collin Dufresne, and Goldstein (2006), Bhamra, Kuhn, and Strebulaev (2008) and most of the papers in the so-called long run risk literature, see Bansal (2009) for a survey.
} 
Despite its importance, the question of endogenous market completeness has not received much attention in the general equilibrium literature. In fact, most of the papers which study multi-asset equilibrium models assume in one form or another that markets are dynamically complete in equilibrium but do not actually prove it. ${ }^{5}$ A notable exception is Anderson and Raimondo (2008) who assume that

(i) The economy has a finite horizon and all traded securities pay strictly positive dividends at the terminal time,

(ii) The state variables of the economy are given by a vector of independent standard Brownian motions,

and prove that under these conditions the candidate prices generate dynamically complete markets as soon as the volatility matrix of the terminal dividends is nondegenerate. Being the first of its kind, the result of Anderson and Raimondo (2008) is obviously very important. However, their assumptions are often too strong to be applicable in practice. For example, assumption (i) requires that all traded assets pay terminal dividends and hence does not allow for securities which pay only continuous dividends as is customary in the continuoustime asset pricing literature. ${ }^{6}$ Furthermore, this assumption implies that the menu of traded securities cannot include instantaneously risk free bonds. The latter restriction is not in itself unnatural as one could for example use a longlived zero coupon bond in zero net supply in place of instantaneously risk free bond and then change numéraire to obtain a truly riskless asset. However, most continuous-time asset pricing models take instantaneously risk free bonds as primitive securities and it is therefore important to find conditions ensuring endogenous market completeness in such economies. Another obvious, but nonetheless important, limitation of assumption (i) is that it does not allow for infinite horizon economies.

While assumption (ii) is satisfied in the benchmark case where dividends are modeled as correlated geometric Brownian motions, Anderson and Raimondo (2008) themselves remark that it is nonetheless quite restrictive. First, this assumption severely restricts the set of dynamics that can be considered by imposing that all economic quantities of interest be functions of time and the

\footnotetext{
${ }^{5}$ See for examples Duffie and Huang (1985), Duffie (1986), Huang (1987), Duffie and Zame (1989) and Dumas, Kurshev, and Uppal (2008) among others.

${ }^{6}$ The assumption of no terminal lump dividends is usually imposed in order to prevent the equilibrium asset prices from being artificially discontinuous at the terminal time due a misalignment between the agents' preferences for intermediate and terminal consumption. See Section 3.1 below for details.
} 
current value of the Brownian motion. In particular, this assumption does not allow for mean reversion in the dynamics of the state variables and thus excludes all of the standard equilibrium term structure models which assume that the state variables follow mean reverting affine processes, see Vasicek (1977) and Cox, Ingersoll, and Ross (1985) among others. Second, and more importantly, this assumption does not make a distinction between state variables and sources of risk and therefore does not allow to consider economies where there are more state variables than independent sources of risk. Important examples of such economies include those where agents have heterogenous beliefs about the evolution of the state variables and, more generally, those where the growth rates and/or volatilities of dividends act as state variables.

In this paper, we extend the result of Anderson and Raimondo (2008) by removing both of their key assumptions. More precisely, we provide sufficient conditions for endogenous market completeness in a continuous-time economy populated by heterogenous agents and driven by an arbitrary multidimensional diffusion process. In our formulation, the traded securities do not need to pay terminal dividends. As a result, the horizon of the economy can be either finite or infinite and we can include instantaneously risk free bonds in the menu of traded assets as is customary in the continuous time asset pricing literature. Another advantage of our formulation over that of Anderson and Raimondo (2008) is that it makes a clear distinction between state variables and sources of risk and thus allows for a much richer set of dynamics. The main results of this paper show that, under suitable regularity conditions on the candidate prices, dynamic market completeness can be deduced from the structure of dividends.

In order to highlight the intuition behind our results consider a finite horizon economy and recall that market completeness hinges on the invertibility of the endogenous volatility matrix of the traded risky securities. The starting point of our analysis is an asymptotic expansion which allows us to compute the volatility matrix of the candidate prices from the primitives of the economy in a neighborhood of the terminal time. Using this expansion we can then easily verify whether markets are dynamically complete in a neighborhood of the terminal time and the question becomes that of finding conditions under which we may propagate this nondegeneracy to the whole interval. As observed by Anderson and Raimondo (2008), who seem to have been the first ones to use it in this context, the notion of real analyticity is particularly well suited to answer this question because a real analytic function which is non zero in an open set is automatically non zero almost everywhere in its domain of analyticity. Applying this result to the problem at hand shows that the volatility 
matrix of the candidate prices is non degenerate at all times provided that it is nondegenerate in a neighborhood of the terminal time and that the candidate prices are real analytic.

When the risky securities pay terminal dividends, the zero order term in the asymptotic expansion of the price volatility is the volatility of the terminal dividends and it follows that markets are complete as soon as this matrix is nondegenerate. In that case our result coincides with that of Anderson and Raimondo (2008) albeit with a slightly different market structure. When the securities do not pay terminal dividends, the asymptotic expansion does not include a zero order term. However, the first order term is proportional to the volatility of the intermediate dividends and it follows that markets are complete as soon as this matrix is nondegenerate. A striking feature of these results is that they only depend on the structure of the dividends of the traded securities: changing the agents' preferences and/or initial endowments has no effect on the existence of an equilibrium.

The requirement that the volatility matrix of the dividends be non degenerate is sufficient to guarantee that markets are endogenously complete in equilibrium but it is by no means necessary. In particular, if some of the traded risky assets are fixed income securities, such as bonds or annuities, then this requirement obviously fails but markets may nonetheless be dynamically complete in equilibrium. In order to obtain sufficient conditions for the existence of an equilibrium in such cases it is necessary to expand the volatility of the candidate prices to higher orders and we provide complete details for the second order expansion. Since some of the securities now draw their value only from variations in the equilibrium pricing kernel, the second order conditions we obtain depend on the agents' preferences and endowments but they are nonetheless quite simple to verify from the primitives of the economy.

In order to apply our results to a given model it is necessary to prove that the candidate prices are real analytic. ${ }^{7}$ While real analyticity in the space variable can be established under rather mild smoothness conditions, real analyticity in time is in general much harder to prove. Furthermore, the conditions that are needed to insure it are often very technical and not really informative. For these reasons we decided to not try and give general conditions and instead focus on two benchmark cases: that where the state variables are given by a vector autoregressive process, and that where they are given by a vector of arbitrary

\footnotetext{
${ }^{7}$ More precisely, we need to show that the candidate prices are not only separately real analytic with respect to time and the state variables but also jointly real analytic. However, we need not worry about this issue in general since separately real analytic functions are under mild conditions jointly real analytic by application of the results of Siciak (1969).
} 
autonomous diffusion processes such as square root or CEV processes. In both cases, we provide joint conditions on the utility functions and dividends under which the prices are real analytic as functions of time and the state variables. Furthermore, we show that if agents have heterogenous power utility functions, as is often the case in the asset pricing literature, then market completeness can be guaranteed by assuming that the dividends have a nondegenerate volatility matrix and satisfy a mild growth conditions.

Our main results show that if the economy has a finite horizon and the candidate prices are real analytic, then dynamic market completeness can be deduced from the structure of dividends provided that the candidate prices are real analytic. Unfortunately, and as explained above, proving that the candidate prices are real analytic is in general difficult and it is therefore natural to wonder whether this inherently technical requirement is crucial for endogenous market completeness. We show that this is indeed the case by constructing explicit examples of representative agent economies where the dividends have a nondegenerate volatility matrix and markets are nonetheless incomplete in equilibrium because the candidate prices are not real analytic.

When the economy has an infinite horizon there is no terminal time close to which the volatility matrix of the candidate prices can be approximated. To circumvent this difficulty we follow an alternative approach: we fix the vector of Pareto weights which determines the consumption allocation and approximate the volatility of the candidate prices as functions of the agents's common rate of time preference. ${ }^{8}$ In other words, we start from a given Pareto optimal allocation and look for conditions under which the corresponding prices generate dynamically complete markets. While it does not deliver market completeness for a fixed set of initial endowments, this alternative approach is nevertheless quite powerful. In particular, it allows us to show that if the volatility of the intermediate dividends is nondegenerate then the candidate prices associated to a Pareto optimal allocation generate dynamically complete markets for all but countably many values of the agents' common rate of time preference. An important advantage of infinite horizon economies over finite horizon ones is that in the former case we do need to assume any real analyticity. The reason for this simplification is that, in an infinite horizon economy, the gradient of the candidate prices is automatically real analytic as a function of the agent's common rate of time preference and this is all that is needed in order to deduce

\footnotetext{
${ }^{8}$ The assumption that agents have a common rate of time preference implies that prices are time independent functions of the state variables and is quite common in infinite horizon economies, see Wang (1996), Chan and Kogan (2002) and Dumas et al. (2008) among others.
} 
market completeness from the structure of dividends.

The rest of the paper is organized as follows. In Section 2 we present the model, state our assumptions and recall some basic results about the existence and characterization of Arrow-Debreu equilibria. Section 3 contains our main sufficient conditions for endogenous market completeness in finite or infinite horizon economies. In Section 4 we use our main results to obtain conditions for market completeness in economies driven by either vector autoregressive processes (Sections 4.1) or vectors of autonomous diffusions (Section 4.2). Section 4.3 presents some counterexamples which show that real analyticity is crucial for market completeness. Section 5 concludes the paper. The proofs of all the results are provided in the appendix.

\section{The economy}

\section{$2.1 \quad$ Information structure}

We consider a continuous-time economy on the time span $[0, T]$ for some horizon $T$ which can be either finite or infinite.

Uncertainty in the economy is represented by a filtered probability space $(\Omega, \mathcal{F}, \mathbb{F}, P)$ supporting a $d$-dimensional standard Brownian motion $Z$. The filtration $\mathbb{F}=\left(\mathcal{F}_{t}\right)_{t \in[0, T]}$ is the usual augmentation of the filtration generated by the Brownian motion, and we let $\mathcal{F} \equiv \mathcal{F}_{T}$ so that the true state of nature is entirely determined by the path of the Brownian motion up to the terminal time of the economy.

\subsection{Securities markets}

The financial market is perfect and consists in $d+1$ traded securities: one locally riskless money market account in zero net supply, and $d$ dividend-paying stocks which are indexed by $i .^{9}$ Stock $i$ is in positive net supply of one unit ${ }^{10}$ and

\footnotetext{
${ }^{9}$ The market structure that we consider, with a locally riskless money market account and $d$ dividend-paying stocks is standard in continuous-time finance, see for example Duffie (2001, Chapter 9) and the references therein. While our analysis can be easily adapted to treat the market structure of Anderson and Raimondo (2008) where none of the securities is riskless we choose to focus on the standard formulation for simplicity of exposition. The results for this alternative market structure are similar to those we present and are available upon request.

${ }^{10}$ The assumption that all risky securities are in positive net supply is only imposed for simplicity of exposition. Our results directly extend to the case where some risky securities are in zero net supply as long as we modify the definition of the aggregate supply of the consumption good to include only the dividends of those securities which are in positive net supply.
} 
represents a claim to a cumulative dividend process of the form ${ }^{11}$

$$
D_{i t} \equiv \int_{0}^{t \wedge T} g_{i}\left(\tau, X_{\tau}\right) d \tau+1_{\{t \geq T\}} G_{i}\left(X_{T}\right)
$$

Here $g_{i} \geq 0$ represents a flow rate of dividends, $G_{i} \geq 0$ represents a terminal lump dividend, and $X \in \mathbb{R}^{n}$ is state variables which evolve according to

$$
X_{t}=X_{0}+\int_{0}^{t} \mu_{X}\left(\tau, X_{\tau}\right) d \tau+\int_{0}^{t} \sigma_{X}\left(\tau, X_{\tau}\right) d Z_{\tau}
$$

for some constant initial value $X_{0} \in \mathbb{R}^{n}$ and some given functions $\mu$ and $\sigma$ with values in $\mathbb{R}^{n}$ and $\mathbb{R}^{n \times d}$. The key conditions that we impose on the dividends and state variables are summarized in the following:

Assumption A: The drift, volatility and dividends are such that

(a) $n \geq d$ and $\operatorname{rank}\left(\sigma_{X}(t, x)\right) \geq d$ for all $(t, x) \in[0, T] \times \mathbb{R}^{n}$,

(b) The stochastic differential equation (1) admits a unique strong solution which takes values in some $\mathcal{X} \subseteq \mathbb{R}^{n}$ and admits an absolutely continuous transition density with respect to Lebesgue measure.

(c) The functions $\mu_{X}$ and $\sigma_{X}$ are real analytic in $(t, x) \in[0, T] \times \mathcal{X} .{ }^{12}$

(d) The function $g_{i}$ is real analytic in $(t, x) \in[0, T] \times \mathcal{X}$ and there exists a open set $\mathcal{V} \subseteq \mathcal{X}$ such that the function $G_{i}$ belongs to $C^{1}(\mathcal{V})$.

The first part of the above assumption implies that the state variables are locally equivalent to the underlying Brownian motions and thus guarantees that the corresponding information structures coincide. If this assumption was not satisfied, then the information carried by the state variables would be strictly contained in that generated by the Brownian motions and, as a result, markets could never be dynamically complete in equilibrium.

The existence of an absolutely continuous transition density is posited in order to guarantee that statements about functions which hold almost everywhere in $[0, T] \times \mathcal{X}$ also hold almost surely when $x$ is replaced by an arbitrary realization of the state variables. Sufficient conditions for the validity of this

\footnotetext{
${ }^{11}$ If the economy has an infinite horizon then we naturally assume that stocks do not pay terminal dividends and set $G_{i} \equiv 0$ for all $i$.

${ }^{12}$ A function $F:[0, T] \times \mathcal{X} \rightarrow \mathbb{R}$ is real analytic in $(t, x)$ over a subset $\mathcal{O} \subseteq[0, T] \times \mathcal{X}$ if for every $(t, x) \in \mathcal{O}$ it can be represented as a convergent power series on some open ball of strictly positive radius centered at $(t, x)$. See Krantz and Parks (2002) for a thorough treatment of the theory of real analytic functions of several variables.
} 
rather weak assumption can be found in numerous references including Stroock and Varadhan (1979) and Nualart (2006) among others.

The assumption that the drift, volatility and intermediate dividends are real analytic does not impose any serious economic restriction since most, if not all, of the models in the literature satisfy these conditions. Importantly, we do not require that the terminal dividends be real analytic or even differentiable on the whole state space. As in Anderson and Raimondo (2008) this generality allows us to include derivatives with non differentiable payoffs, such as options, in the menu of traded risky securities.

In what follows we denote by $\mathbb{P}=(B, S) \in \mathbb{R}^{1+d}$ the endogenous equilibrium prices of the riskless asset and the stocks. As shown below, there is no loss in generality in assuming that these processes are strictly positive semimartingales such that $B$ is of finite variation and satisfies $B_{0} \equiv 1$.

\subsection{Consumption, preferences and endowments}

The consumption space is the set of pairs $(c, C)$ where $c$ is a nonnegative adapted process which represents a flow rate of consumption, and $C$ is a nonnegative random variable which represents a terminal lumpsum consumption.

The economy is populated by $A \geq 1$ agents indexed by $a$. The preferences of agent $a$ over lifetime consumption plans are represented by a time additive expected utility index of the form ${ }^{13}$

$$
U_{a}(c, C) \equiv E_{0}\left[\int_{0}^{T} e^{-\rho_{a} \tau} u_{a}\left(c_{\tau}\right) d \tau+e^{-\rho_{a} T} v_{a}(C)\right] .
$$

In the above equation, the constant $\rho_{a} \geq 0$ is the agent's subjective rate of time preferences and $\left(u_{a}, v_{a}\right)$ is a pair of von Neuman-Morgenstern utility functions which satisfy the following assumption. ${ }^{14}$

Assumption B: The utility functions $u_{a}, v_{a}:(0, \infty) \rightarrow \mathbb{R}$ are increasing, strictly concave, real analytic and satisfy the Inada conditions $u_{a}^{\prime}(0)=v_{a}^{\prime}(0)=\infty$ and $u_{a}^{\prime}(\infty)=v_{a}^{\prime}(\infty)=0$.

Agent $a$ is initially endowed with a portfolio consisting of $\eta_{a i}$ units of stock

\footnotetext{
${ }^{13}$ If the economy has an infinite horizon, or if there are no terminal dividends then we assume that agents have no utility for terminal consumption and set $v_{a} \equiv 0$ for all $a$.

${ }^{14}$ All of our analysis can be extended to allow for utility functions which depend on the state of nature through the state variables of the model as in Anderson and Raimondo (2008). We restrict ourselves to the state independent case for simplicity of exposition.
} 
$i$, and we assume that

$$
\sum_{a=1}^{A} \eta_{a i}=1
$$

for each $i$ so that the securities markets clear at the initial date. ${ }^{15}$ Initial short positions in some of the stocks are allowed as long as the initial wealth of agent $a$, which is defined by

$$
w_{a} \equiv \eta_{a}^{\top} S_{0}=\sum_{i=1}^{d} \eta_{a i} S_{i 0},
$$

is strictly positive when computed at equilibrium prices.

\subsection{Trading strategies and feasible plans}

A trading strategy is an adapted process $(\alpha, \pi) \in \mathbb{R}^{1+d}$ where $\alpha_{t}$ and $\pi_{i t}$ denote, respectively, the number of units of the riskless asset and the number of units of stock $i$ which are held in the portfolio at time $t$.

A trading strategy $(\alpha, \pi)$ is said to finance a consumption plan $(c, C)$ at cost $w$ if the associated wealth process

$$
W_{t} \equiv \alpha_{t} B_{t}+\pi_{t}^{\top} S_{t}
$$

is nonnegative and satisfies the dynamic budget constraint

$$
W_{t}=w+\int_{0}^{t} \alpha_{\tau} d B_{\tau}+\int_{0}^{t} \pi_{\tau}^{\top} d(S+D)_{\tau}-\int_{0}^{t} c_{\tau} d \tau-1_{\{t \geq T\}} C
$$

where $D \in \mathbb{R}^{d}$ denotes the vector of cumulative dividends. ${ }^{16}$ In what follows, a consumption plan is said to be feasible for agent $a$ if there exists a trading strategy which finances it at cost $w_{a}=\eta_{a}^{\top} S_{0}$, and we let $\mathcal{C}_{a}(B, S)$ denote the corresponding set of budget feasible consumption plans.

\footnotetext{
${ }^{15}$ For simplicity of exposition, we do not include individual income processes on top of the agents' initial portfolios. However, all of our analysis can be easily extended to that case as long as the individual income processes are modeled as functions of time and the state variables of the model.

${ }^{16}$ The requirement that the wealth process satisfies equation (2) implicitly includes the condition that the various integrals are well defined. The necessary integrability conditions are well-known and can be found in standard textbooks such as Duffie (2001)
} 


\subsection{Equilibrium}

The concept of equilibrium that we use is similar to that of equilibrium of plans, prices and expectations introduced by Radner (1972).

Definition 1: An equilibrium consists of a pair $\mathbb{P}=(B, S)$ of securities price processes, a consumption allocation $\mathbb{A}=\left\{\left(c_{a}, C_{a}\right)\right\}_{a=1}^{A}$ and a set of trading strategies $\mathbb{S}=\left\{\left(\alpha_{a}, \pi_{a}\right)\right\}_{a=1}^{A}$ such that:

(a) For each a the consumption plan $\left(c_{a}, C_{a}\right)$ maximizes $U_{a}$ over $\mathcal{C}_{a}(B, S)$ and is financed by $\left(\alpha_{a}, \pi_{a}\right)$.

(b) Markets clear in the sense that $\sum_{a=1}^{A} \alpha_{a t}=0, \sum_{a=1}^{A} \pi_{a t}=\mathbf{1}$, and

$$
\sum_{a=1}^{A}\left(c_{a t}-\eta_{a}^{\top} g\left(t, X_{t}\right)\right)=\sum_{a=1}^{A}\left(C_{a}-\eta_{a}^{\top} G\left(X_{T}\right)\right)=0
$$

hold almost surely for all $t \in[0, T)$ where $g \in \mathbb{R}^{d}$ and $G \in \mathbb{R}^{d}$ denote the vectors of intermediate and terminal dividends.

An equilibrium is said to have dynamically complete markets if, subject to a mild integrability condition, any consumption plan can be financed by some trading strategy at some finite initial cost.

The rest of the paper is devoted to finding conditions under which there exists an equilibrium with dynamically complete markets. As explained in the next section, the starting point of our analysis will be a static Arrow-Debreu equilibrium which we define as follows:

Definition 2: An Arrow-Debreu equilibrium is a nonnegative consumption price process $m$ and a consumption allocation $\mathbb{A}=\left\{\left(c_{a}, C_{a}\right)\right\}_{a=1}^{A}$ such that

(a) For each a the pair $\left(c_{a}, C_{a}\right)$ maximizes $U_{a}$ over the set of consumption plans which satisfy the static budget constraint

$$
E_{0}\left[\int_{0}^{T} m_{\tau}\left(c_{\tau}-\eta_{a}^{\top} g\left(\tau, X_{\tau}\right)\right) d \tau+m_{T}\left(C-\eta_{a}^{\top} G\left(X_{T}\right)\right)\right] \leq 0 .
$$

(b) The consumption good market clears in the sense that equation (3) holds almost surely for all $t \in[0, T)$.

Assumption C: The set of Arrow-Debreu equilibria is non empty. 
Various sets of sufficient conditions for the validity of the above assumption can be found in the literature, see for example Duffie and Zame (1989), Karatzas et al. (1990) and Mas Colell and Zame (1991) among others. In a recent paper Malamud (2008) obtained simple necessary and sufficient conditions for the existence of an Arrow-Debreu equilibrium in an economy populated with constant relative risk aversion agents. A slight modification of his arguments delivers the following general result:

Proposition 1: Assume that for each a there are strictly positive constants $\gamma_{a 1}$ and $\gamma_{a 2}$ such that

$$
\gamma_{a 1} \leq-\frac{x u_{a}^{\prime \prime}(x)}{u_{a}^{\prime}(x)},-\frac{x v_{a}^{\prime \prime}(x)}{v_{a}^{\prime}(x)} \leq \gamma_{a 2}
$$

and

$$
\sum_{a=1}^{A} \sum_{n=1}^{2} E_{0}\left[\int_{0}^{T} e^{-\rho_{a} \tau} \bar{g}\left(\tau, X_{\tau}\right)^{1-\gamma_{a n}} d \tau+e^{-\rho_{a} T} \bar{G}\left(X_{T}\right)^{1-\gamma_{a n}}\right]<\infty,
$$

where the functions $\bar{g} \equiv g^{\top} \mathbf{1}$ and $\bar{G} \equiv G^{\top} \mathbf{1}$ denote the aggregate dividend rate and the aggregate terminal dividend. Then Assumption $C$ holds.

The following well-known result (see Huang (1987) or Duffie (2001) among others) provides an explicit characterization of the equilibrium consumption price process as the marginal utility of a representative agent endowed with the aggregate supply of the consumption good, and will be useful for our construction of equilibrium prices in the next section.

Proposition 2: In an Arrow-Debreu equilibrium

$$
m_{t}=1_{\{t<T\}} \frac{\partial u}{\partial c}\left(t, \lambda, \bar{g}\left(t, X_{t}\right)\right)+1_{\{t=T\}} \frac{\partial v}{\partial c}\left(\lambda, \bar{G}\left(X_{T}\right)\right)
$$

for some vector $\lambda \in \mathcal{S}$ of strictly positive Pareto weights where the social welfare functions are defined by

$$
\begin{aligned}
v(\lambda, c) & \equiv \max _{\alpha \in \mathcal{S}} \sum_{a=1}^{A} \lambda_{a} e^{-\rho_{a} T} v_{a}\left(\alpha_{a} c\right), \\
u(t, \lambda, c) & \equiv \max _{\alpha \in \mathcal{S}} \sum_{a=1}^{A} \lambda_{a} e^{-\rho_{a} t} u_{a}\left(\alpha_{a} c\right),
\end{aligned}
$$

and $\mathcal{S}$ denotes the unit simplex. In particular, the equilibrium consumption price function $m$ is real analytic in $(t, x) \in(0, T) \times \mathcal{X} .{ }^{17}$

\footnotetext{
${ }^{17}$ In the case where the traded security do not pay dividends, the statement can be
} 
As shown by the above result, the equilibrium consumption price process is continuous on $[0, T)$ but has a predictable jump

$$
\Delta\left(X_{T}\right) \equiv \frac{\partial v}{\partial c}\left(\lambda, \bar{G}\left(X_{T}\right)\right)-\frac{\partial u}{\partial c}\left(T, \lambda, \bar{g}\left(T, X_{T}\right)\right)
$$

at the terminal time. In the context of our model, this somehow unnatural jump reflects the potential misalignment between the representative agents' preferences for intermediate and terminal consumption on the one hand, and between intermediate and terminal dividends on the other. ${ }^{18}$ It can be avoided by assuming that either dividends and utilities are aligned in the sense that $\bar{g}(T, x)=\bar{G}(x)$ and $u_{a}(c)=v_{a}(c)$ for each $a$, or that the stocks do not pay lumpsum dividends at the terminal date.

\section{Endogenous completeness}

In order to find conditions under which our economy admits an equilibrium with dynamically complete markets, we will follow the path set by Kreps (1982), Duffie and Huang (1985), Duffie (1986) and Huang (1987). Namely, we will start from a given Arrow-Debreu equilibrium, then construct candidate prices for the traded securities by using the consumption price process as a state price deflator, and finally check whether these prices deliver complete markets.

\subsection{Candidate price functions}

Fix an arbitrary Arrow-Debreu equilibrium and let $m_{t} \equiv m\left(t, X_{t}\right)$ denote the corresponding consumption price process. Appealing to Proposition 2 for the required smoothness and applying Itô's lemma we obtain

$$
d m_{t}=-m_{t-} d R_{t}+\frac{\partial m}{\partial x}\left(t, X_{t}\right)^{\top} \sigma\left(t, X_{t}\right) d Z_{t}
$$

where the finite variation process $R$ is defined by

$$
R_{t}=-\int_{0}^{t \wedge T} \frac{\mathcal{D} m\left(\tau, X_{\tau}\right)}{m\left(\tau, X_{\tau}\right)} d \tau+1_{\{t \geq T\}} \log \left(1-\frac{\Delta\left(X_{T}\right)}{m\left(T, X_{T}\right)}\right)
$$

strengthened to show that $m$ is real analytic in $(t, x) \in(0, T] \times \mathcal{X}$.

${ }^{18}$ Other examples of equilibrium models where prices have singular components include the case of finite marginal utility at zero studied by Karatzas, Lehoczky, and Shreve (1991), the liquidity constraints model of Detemple and Serrat (2003) and the portfolio insurance model of Basak (1995) among others. 
and $\mathcal{D} \equiv \partial / \partial t+\mathcal{L}$ denotes the extended infinitesimal generator of the state variables. Equation (6) shows that $-d R_{t}$ measures instantaneous expected percentage changes in the marginal rates of substitution of the representative agent along the equilibrium path, and so we will take $B \equiv \exp (R)$ as our candidate for the equilibrium price of the riskless asset.

On the other hand, a natural candidate for the equilibrium price of the stocks is given by the fundamental value of the dividends computed at the equilibrium price of the consumption good, namely

$$
S_{t}=1_{\{t<T\}} P\left(t, X_{t}\right)+1_{\{t=T\}} G\left(X_{T}\right)
$$

where $P$ is the function defined by

$$
P\left(t, X_{t}\right) \equiv E_{t}\left[\int_{t}^{T} \frac{m\left(\tau, X_{\tau}\right)}{m\left(t-, X_{t}\right)} g\left(\tau, X_{\tau}\right) d \tau+\frac{m\left(T, X_{T}\right)}{m\left(t-, X_{t}\right)} G\left(X_{T}\right)\right] .
$$

As can be seen from equations (7), (8) and (9), the candidate price processes are continuous on $[0, T)$ and have a jump at the terminal time which they inherit from the discontinuity in the equilibrium price of the consumption good. However, and as required to guarantee that prices are arbitrage-free, the relative jumps on all traded securities are equal:

$$
\lim _{t \rightarrow T} \frac{B_{T}}{B_{t}}=\lim _{t \rightarrow T} \frac{S_{i T}}{S_{i t}}=Q\left(X_{T}\right) \equiv 1-\frac{\Delta\left(X_{T}\right)}{m\left(T, X_{T}\right)} .
$$

If this was not the case then trivial arbitrages could be implemented by buying a security with a larger relative jump and short-selling the same amount of another with a smaller relative jump just prior to the terminal time.

In order to assert that the candidate price system $\mathbb{P}=(B, S)$ gives rise to an equilibrium it is sufficient to prove that if the riskless asset and the stocks can be traded at these prices then markets are dynamically complete. In a continuoustime model such as ours, dynamic market completeness of a given price system is closely related to the properties of the volatility matrix of risky securities. More precisely, it can be shown that markets are dynamically complete if and only if the volatility matrix of the stocks is almost everywhere invertible, see for example Duffie (2001, Chapter 6) or Karatzas and Shreve (2001, Chapter 1). Now assume that $P$ is smooth enough for an application of Itô's lemma. In that case the volatility matrix of the candidate prices can be computed as

$$
\sigma_{P}\left(t, X_{t}\right) \equiv \frac{\partial P}{\partial x}\left(t, X_{t}\right) \sigma_{X}\left(t, X_{t}\right)
$$


and we conclude that markets are dynamically complete if and only if the matrix valued function $\sigma_{P}$ is nondegenerate. The following proposition summarizes the above discussion.

Proposition 3: Fix an Arrow-Debreu equilibrium $(m, \mathbb{A})$ and let the price system $\mathbb{P}=(B, S)$ be defined as above. If $P \in C^{1,2}((0, T) \times \mathcal{X})$ and

$$
\operatorname{det} \sigma_{P}(t, x) \neq 0
$$

almost everywhere on $(0, T) \times \mathcal{X}$ then there exists a set $\mathbb{S}$ of strategies such that $\mathbb{P}, \mathbb{A}$ and $\mathbb{S}$ form an equilibrium with dynamically complete markets.

The main obstacle one encounters when trying to apply the above result in order to establish the existence of an equilibrium is that, unless $d=1$ or the candidate price function can be computed in closed form, ${ }^{19}$ it is in general very difficult to check directly that equation (10) holds. To circumvent this difficulty, we show in the next section that, under appropriate regularity conditions, it may be sufficient to check that the volatility of the dividends, rather than that of the price itself, is nondegenerate.

\subsection{Real analyticity and market completeness}

In this section we present conditions which are sufficient to guarantee that the gradient of the candidate price function is invertible and, hence, that there exists an equilibrium with dynamically complete markets. In order to highlight the intuition behind our approach we start by considering the finite horizon case before we turn to infinite horizon economies.

\subsubsection{Finite horizon economies}

Let $T$ be a finite horizon and $P$ be the pre-horizon price function defined by equation (9). This function satisfies

$$
\lim _{t \rightarrow T} P(t, x)=\frac{G(x)}{Q(x)} \equiv \mathcal{G}(x)
$$

and, assuming that it is once continuously differentiable with respect to the space variable for all $(t, x) \in(0, T] \times \mathcal{V}$, we obtain

$$
\frac{\partial P}{\partial x}(t, x)=\frac{\partial \mathcal{G}}{\partial x}(x)+o(1) .
$$

\footnotetext{
${ }^{19}$ Examples of multi-asset equilibrium models where the candidate price function can be computed in closed form include those of Santos and Veronesi (2006), Cochrane et al. (2008), Hugonnier (2008) and Martin (2008) among others.
} 
This equation shows that sufficiently close to the terminal time, the volatility of the candidate stock prices can be approximated by that of the vector $\mathcal{G}\left(X_{t}\right)$ of "effective" terminal dividends. In particular, the corresponding determinants are related by

$$
\operatorname{det} \sigma_{P}(t, x)=\operatorname{det} \sigma_{\mathcal{G}}(T, x)+o(1)
$$

for all $x \in \mathcal{V}$ where

$$
\sigma_{\mathcal{G}}(t, x) \equiv \frac{\partial \mathcal{G}}{\partial x}(x) \sigma_{X}(t, x),
$$

denotes the volatility of the effective terminal dividends; and it follows that equation (10) holds for almost every $x \in \mathcal{V}$ in a neighborhood of the terminal time as soon as $\sigma_{\mathcal{G}}(T, x)$ is non degenerate for almost every $x \in \mathcal{V}$.

Assuming that the above calculations can be justified, the question becomes that of knowing whether there are conditions under which we may propagate the validity of equation (10) from a open subset of $(0, T) \times \mathcal{X}$ to the whole set. As observed by Anderson and Raimondo (2008) who seem to have been the first ones to use it in this context, the notion of real analyticity is particularly well suited to answer this question because a real analytic function which is non zero on an open set is automatically non zero almost everywhere in its domain of analyticity. Applying this simple property to the problem at hand gives us our first main result:

TheOrem 1: Assume that the function $P$ of equation (9) is real analytic in $(t, x) \in(0, T) \times \mathcal{X}$ and belongs to $C^{1}((0, T] \times \mathcal{V})$. If

$$
\operatorname{det} \sigma_{\mathcal{G}}(T, x) \neq 0
$$

holds for almost every $x \in \mathcal{V}$ then there exists an equilibrium with dynamically complete markets.

The above theorem shows that if the price function is real analytic then dynamic market completeness obtains as soon as the volatility of $\mathcal{G}$ is nondegenerate. If one assumes, in addition, that there is no jump in the equilibrium consumption price process at the terminal time, then this condition simplifies further and only requires that the volatility matrix

$$
\sigma_{G}(t, x) \equiv \frac{\partial G}{\partial x}(x) \sigma_{X}(t, x)
$$

of the terminal dividends be nondegenerate. In that case, our condition is 
similar to that obtained by Anderson and Raimondo (2008) albeit with a slightly different market structure. ${ }^{20}$ However, it is important to note that our result allows for more state variables than there are underlying sources of risk and does not require that these state variables be Brownian motions.

Most of the asset pricing literature focuses on equilibrium models in which risky securities do not pay terminal dividends. For such models, Theorem 1 is of no help since the sufficient conditions it provides require that all the risky securities pay terminal dividends. Nevertheless, and as we now demonstrate, the techniques used to establish Theorem 1 can be easily adapted to cover the case of risky securities which only pay intermediate dividends.

Assume for simplicity that agents have no utility for terminal consumption, and that the securities do not pay terminal dividends. In that case the equilibrium consumption price process is continuous and, as a result, the candidate price function is simply defined by

$$
S_{t}=P\left(t, X_{t}\right)=E_{t}\left[\int_{t}^{T} \frac{m\left(\tau, X_{\tau}\right)}{m\left(t, X_{t}\right)} g\left(\tau, X_{\tau}\right) d \tau\right] .
$$

Using this expression and assuming that the interchange of limit and expectation can be justified, we obtain

$$
\begin{aligned}
-\frac{\partial P}{\partial t}(T, x) & =\lim _{t \uparrow T} \frac{P(t, x)-P(T, x)}{T-t} \\
& =\lim _{t \uparrow T} E_{t}\left[\frac{1}{T-t} \int_{t}^{T} \frac{m\left(\tau, X_{\tau}\right)}{m(t, x)} g\left(\tau, X_{\tau}\right) d s\right]=g(T, x),
\end{aligned}
$$

and it follows that

$$
P(t, x)=(T-t) g(T, x)+o(T-t)
$$

Assuming further that the relevant cross-derivative is continuous all the way to the terminal time, and formally differentiating on both sides of the above expression we obtain that the volatility of the candidate prices satisfies

$$
\sigma_{P}(t, x)=(T-t) \sigma_{g}(T, x)+o(T-t)
$$

\footnotetext{
${ }^{20}$ Anderson and Raimondo (2008) assume that in place of instantaneously riskfree bonds the menu of traded securities includes a zero net supply security with terminal payoff $G_{0}>0$ and price $P_{0}$. In that case a straightforward modification of our arguments shows that Theorem 1 remains valid provided that we replace $P$ by $P / P_{0}$ and $\mathcal{G}$ by $G / G_{0}$.
} 
where

$$
\sigma_{g}(t, x) \equiv \frac{\partial g}{\partial x}(t, x) \sigma_{X}(t, x)
$$

is the volatility of the intermediate dividends. This equation shows that, in a neighborhood of the terminal time, the volatility of the candidate prices is proportional to that of the intermediate dividends. In particular, equation (10) holds almost everywhere in a neighborhood of the terminal time as soon as the matrix $\sigma_{g}(T, x)$ is non degenerate for almost every $x \in \mathcal{X}$, and assuming that the candidate price function is real analytic then allows to conclude that markets are complete. We summarize the above discussion as follows:

Theorem 2: Assume that the function $P$ of equation (12) is real analytic in $(t, x) \in(0, T) \times \mathcal{X}$ and belongs to $C^{2}((0, T] \times \mathcal{X})$. If

$$
\operatorname{det} \sigma_{g}(T, x) \neq 0
$$

holds for almost every $x \in \mathcal{X}$ then there exists an equilibrium with dynamically complete markets.

The results of the above theorems are quite intuitive. Indeed, these theorems simply state that if the price function is real analytic in time then non degeneracy of the volatility of either terminal or intermediate dividends is automatically transmitted to the volatility matrix of the prices themselves. A striking feature of these results is that they depend only on the dividends and not on the other primitives of the economy: changing the agents' preferences or initial endowments has no effect on the existence of an equilibrium. If one can prove real analyticity of the price function from first principles, then Theorems 1 and 2 provide an easy way of deciding whether a given model gives rise to equilibrium with dynamically complete markets by simply checking if the dividends are functionally independent. As we illustrate in Section 4 below, the most difficult part in this line of attack is of course to find reasonably general conditions which guarantee that the candidate price function is real analytic.

The conditions of Theorems 1 and 2 are sufficient to guarantee the existence of an equilibrium with dynamically complete markets but they are by no means necessary. In particular, if some of the risky assets are fixed income securities which pay constant dividends, then equation (13) obviously fails but markets may nonetheless be dynamically complete as illustrated by Example 1 below. In order to find sufficient conditions for dynamic market completeness in such cases one needs to perform a higher order expansion of the volatility of the candidate 
prices. While such an expansion can in principle be computed to an arbitrary order, the derived expression become more and more complicated as the order of the expansion increases. For the sake of readability, we therefore present the details only for the second order expansion.

Under appropriate regularity conditions, it can be shown that a second order expansion of the gradient of the price function is given by

$$
\sigma_{P}(t, x)=(T-t) \sigma_{g}(T, x)+\frac{1}{2}(T-t)^{2} H(x)+o(T-t)^{2}
$$

where we have set

$$
H(x)=\frac{\partial}{\partial x}\left(\frac{\mathcal{D}(m g)(T, x)}{m(T, x)}\right) \sigma_{X}(T, x)-2 \frac{\partial \sigma_{g}}{\partial t}(T, x)
$$

and $\mathcal{D} \equiv \partial / \partial t+\mathcal{L}$ denotes the extended infinitesimal generator of the state variables. Combining this expansion with well-known results from linear algebra (see the appendix for details) and appealing to the same property of real analytic functions as before leads to a second order sufficient condition for the existence of an equilibrium with dynamically complete markets. In order to state this condition let $B_{i}$ denote the matrix obtained from $\sigma_{g}$ by replacing its $i$-th row with the corresponding row of $H$, namely

$$
B_{i}(x)=\sigma_{g}(T, x)+\mathbf{e}_{i} \mathbf{e}_{i}^{\top}\left(H(x)-\sigma_{g}(T, x)\right),
$$

where $\mathbf{e}_{i}$ is the $i$-th vector in the orthonormal basis of $\mathbb{R}^{d}$.

Proposition 4: Assume that the function $P$ of equation (12) is real analytic in $(t, x) \in(0, T) \times \mathcal{X}$ and belongs to $C^{3}((0, T] \times \mathcal{X})$. If

$$
\sum_{i=1}^{d} \operatorname{det} B_{i}(x) \neq 0
$$

holds for almost every $x \in \mathcal{X}$ then there exists an equilibrium with dynamically complete markets.

In contrast to that of Theorem 2 the sufficient condition of Proposition 4 depends not only on the dividends but also on the agents' preferences and endowments through the equilibrium price of the consumption good. This is intuitive since some of the risky securities now draw their value solely from variations in the equilibrium consumption price process and the latter is jointly determined by dividends, preferences and endowments. Nevertheless, and as we 
illustrate in the following example, the fact that the sum of determinants in equation (15) does not vanish can often be verified by direct calculation.

EXAMPLE 1: Assume that the uncertainty is generated by a two dimensional Brownian motion $Z$ and consider a finite horizon economy populated by identical agents with constant relative risk aversion $\gamma>0$, rate of time preference $\rho \geq 0$ and initial portfolio $\eta=\mathbf{1} / A$.

Assume that the economy is driven by two state variables which evolve according to

$$
\begin{aligned}
& d X_{1 t}=\left(X_{2 t}-\left\|\sigma_{1}\right\|^{2} / 2\right) d t+\sigma_{1}^{\top} d Z_{t} \\
& d X_{2 t}=\kappa\left(\theta-X_{2 t}\right) d t+\sigma_{2}^{\top} d Z_{t}
\end{aligned}
$$

for some $\left(\kappa, \theta, \sigma_{1}, \sigma_{2}\right) \in \mathbb{R} \times \mathbb{R} \times \mathbb{R}^{2} \times \mathbb{R}^{2}$ such that $\sigma_{1}$ and $\sigma_{2}$ are linearly independent; and that the intermediate dividends are given by

$$
\begin{aligned}
& g_{1}\left(t, X_{t}\right) \equiv 1, \\
& g_{2}\left(t, X_{t}\right) \equiv \exp \left(X_{1 t}\right) .
\end{aligned}
$$

The interpretation of this market structure is as follows. The first risky security is an annuity which pays one unit of consumption per unit of time while the second risky security is a stock. The dividends of this stock follow a geometric Brownian motion with constant volatility $\sigma_{1}$ and a stochastic drift which is imperfectly correlated to the dividend and assumed to follow a mean reverting Ornstein-Uhlenbeck process with constant coefficients $\kappa, \theta$ and $\sigma_{2}$.

Using standard results it can be shown that the primitives of this economy satisfy the conditions of Proposition 1 and it follows that an Arrow-Debreu equilibrium exists. However, the conditions of Theorem 2 cannot be applied to assert the existence of an equilibrium with dynamically complete markets since all the derivatives of the function $g_{1}$ are zero. To circumvent this difficulty we will use the result of Proposition 4.

Since agents have homogenous power utility functions, time preference rates and endowments, we know that the equilibrium consumption price process takes the form

$$
m\left(t, X_{t}\right)=e^{-\rho t} A^{\gamma-1}\left(1+\exp \left(X_{1 t}\right)\right)^{-\gamma}
$$

and a straightforward calculation shows that the sum of determinants in equation 
(15) is explicitly given by

$$
\sum_{i=1}^{2} \operatorname{det} B_{i}(x)=\frac{\gamma e^{2 x_{1}}}{1+e^{x_{1}}} \operatorname{det}\left(\sigma_{1}, \sigma_{2}\right) \neq 0 .
$$

Furthermore, it can be checked that the primitives of the model satisfy the conditions of Proposition 6 below and it follows that there exists an equilibrium with dynamically complete markets.

\subsubsection{Infinite horizon economies}

Set $v_{a}=\bar{G} \equiv 0$ for all $a$ and consider an infinite horizon economy. The following assumption guarantees that the candidate prices are time independent functions of the state variables and is quite common in the continuous-time general equilibrium literature, see Wang (1996), Chan and Kogan (2002) and Dumas et al. (2008) among others. ${ }^{21}$

Assumption D: All agents have the same subjective rate of time preference and the functions $\mu, \sigma$ and $g$ are time independent.

Since the model now has an infinite horizon, there is no terminal time close to which the volatility of candidate prices can be approximated. As a result, we cannot use the approach of the preceding section to obtain conditions which guarantee market completeness for a given set of initial endowments. To circumvent this difficulty we will follow an alternative approach: we will fix the vector of Pareto weights which determines the consumption allocation and approximate the candidate prices as functions of the agents's common rate of time preference.

In order to implement this approach, fix an arbitrary vector $\lambda \in \mathcal{S}$ of Pareto weights and denote by $\rho \geq 0$ the agents' common rate of time preference. Using Assumption D together with the result of Proposition 2 it can be shown that the equilibrium consumption price process is given by

$$
m\left(t, X_{t}, \rho, \lambda\right) \equiv e^{-\rho t} \mathbf{m}\left(X_{t}, \lambda\right)
$$

for some function $\mathbf{m}$ which depends only on the state variables and the vector Pareto weights. Given this consumption price process, the candidate stock prices

\footnotetext{
${ }^{21}$ The results of this section can be easily extended to allow for heterogenous discount rates and time dependent dividends at the cost of increased notational burden.
} 
are defined by

$$
\begin{aligned}
S_{t} & =P\left(X_{t}, \rho, \lambda\right) \\
& \equiv E_{t} \int_{t}^{\infty} e^{-\rho(\tau-t)} \frac{\mathbf{m}\left(X_{\tau}, \lambda\right)}{\mathbf{m}\left(X_{t}, \lambda\right)} g\left(X_{\tau}\right) d \tau=\int_{0}^{\infty} e^{-\rho \tau} \frac{\pi\left(\tau, X_{t}, \lambda\right)}{\mathbf{m}\left(X_{t}, \lambda\right)} d \tau
\end{aligned}
$$

where we have set

$$
\pi(\tau, x, \lambda) \equiv E\left[\mathbf{m}\left(X_{\tau}, \lambda\right) g\left(X_{\tau}\right) \mid X_{0}=x\right]
$$

and the last equality follows from the Markov property of the state variables and Fubini's theorem. Since the agents' common rate of time preference only appears in the discount factor, we have that the function $P$ and all its space derivatives are automatically real analytic in $\rho$ as soon as they are well-defined. In order to guarantee that this is indeed the case we following regularity condition:

Assumption E: The function $\pi$ belongs to $C^{2}\left(\mathbb{R}_{+} \times \mathcal{X}\right)$ and there exist a constant $K>0$ and a continuous function $\Pi \geq 0$ such that

$$
\pi_{i}(\tau, x, \lambda)+\left|\frac{\partial \pi_{i}}{\partial x_{j}}(\tau, x, \lambda)\right|+\left|\frac{\partial^{2} \pi_{i}}{\partial x_{j} \partial x_{k}}(\tau, x, \lambda)\right| \leq e^{\tau K} \Pi(x)
$$

for all $(\tau, x) \in(0, \infty) \times \mathcal{X}$ and $1 \leq i, j, k \leq d$.

As a result of the above assumption we have that the candidate price function is well-defined and twice continuously differentiable in with respect to the state variables for all $\rho>K$. Furthermore, it can be shown that the gradient of the candidate price function is real analytic in $\rho$ and satisfies

$$
\frac{\partial P}{\partial x}(x, \rho, \lambda)=(1 / \rho) \frac{\partial g}{\partial x}(x)+o(1 / \rho) .
$$

This equation shows that for large values of $\rho$ the volatility of the candidate prices is approximately proportional to that of the intermediate dividends. In particular, the corresponding determinants are related by

$$
\operatorname{det} \sigma_{P}(x, \rho, \lambda)=(1 / \rho)^{d} \operatorname{det} \sigma_{g}(x)+o(1 / \rho)^{d},
$$

and it follows that the volatility of the candidate prices is invertible almost everywhere in a neighborhood of $1 / \rho=0$ as soon as the volatility of the intermediate dividends is almost everywhere nondegenerate. Since the gradient of the candidate price function is real analytic in $\rho$ we can then propagate this property from a neighborhood of infinity to almost every value of $\rho>K$ and thus 
obtain dynamic market completeness for almost every rate of time preference.

The following result constitutes the exact counterpart of Theorem 2 for the case of infinite horizon economies and summarizes the above discussion.

Theorem 3: Consider an infinite horizon economy and fix a vector of Pareto weights $\lambda \in \mathcal{S}$ such that Assumption E holds true. If

$$
\operatorname{det} \sigma_{g}(x) \neq 0
$$

holds for almost every $x \in \mathcal{X}$ then the consumption allocation associated with $\lambda \in \mathcal{S}$ gives rise to an equilibrium with dynamically complete markets for all but countably many $\rho>K$.

The above result shows that, in an infinite horizon economy, a Pareto optimal allocation gives rise to dynamically complete markets for almost every rate of time preference as soon as the the volatility matrix of the dividends is nondegenerate. As in the finite horizon case, this first order condition is only sufficient and higher order conditions can be obtained by expanding the volatility of the candidate prices further. In particular, it can be shown that the second order expansion is given by

$$
\sigma_{P}(x, \rho, \lambda)=(1 / \rho) \sigma_{g}(x)+(1 / \rho)^{2} F(x, \lambda)+o(1 / \rho)^{2}
$$

where we have set

$$
F(x, \lambda) \equiv \frac{\partial}{\partial x}\left(\frac{\mathcal{L}(\mathbf{m} g)(x, \lambda)}{\mathbf{m}(x, \lambda)}\right) \sigma_{X}(x)
$$

Combining this expression with well-known results from linear algebra and appealing to the same property of real analytic functions as before then leads to a second order sufficient conditions for the existence of an equilibrium with complete markets. In order to state this condition let $C_{i}: \mathcal{X} \times \mathcal{S} \rightarrow \mathbb{R}^{d \times d}$ denote the matrix valued function defined by

$$
C_{i}(x, \lambda) \equiv \sigma_{g}(x)+\mathbf{e}_{i} \mathbf{e}_{i}^{\top}\left(F(x, \lambda)-\sigma_{g}(x)\right)
$$

where $\mathbf{e}_{i}$ is the $i$-th vector in the orthonormal basis of $\mathbb{R}^{d}$.

Proposition 5: Consider an infinite horizon economy and fix a vector of Pareto weights $\lambda \in \mathcal{S}$ such that Assumption E holds true. If

$$
\sum_{i=1}^{d} \operatorname{det} C_{i}(x, \lambda) \neq 0
$$


holds for almost every $x \in \mathcal{X}$ then the consumption allocation associated with $\lambda \in \mathcal{S}$ gives rise to an equilibrium with dynamically complete markets for all but countably many $\rho>K$.

In order to illustrate the application of the above results we now consider an example taken from Dumas et al. (2008).

EXAMPle 2: Assume that the uncertainty is generated by a two dimensional Brownian motion $Z$ and consider an infinite horizon economy driven by a four dimensional vector of state variables which evolves according to

$$
\begin{aligned}
& d X_{1 t}=X_{1 t} X_{2 t} d t+X_{1 t} \sigma_{1} d Z_{1 t} \\
& d X_{2 t}=\kappa\left(\theta-X_{2 t}\right) d t+\sigma_{2} d Z_{1 t} \\
& d X_{3 t}=\kappa\left(\theta-X_{3 t}\right) d t+\sigma_{31} d Z_{1 t}+\sigma_{32} d Z_{2 t} \\
& d X_{4 t}=X_{4 t}\left(\left(X_{3 t}-X_{2 t}\right) / \sigma_{1}\right) d Z_{1 t}
\end{aligned}
$$

for some constants $\left(\kappa, \theta, \psi, \sigma_{1}, \sigma_{2}, \sigma_{3}\right) \in \mathbb{R} \times \mathbb{R} \times \mathbb{R} \times \mathbb{R} \times \mathbb{R} \times \mathbb{R}^{2}$ such that $\sigma_{32} \neq 0$, $\sigma_{1} \neq 0$. The economy is populated with two agents whose utility functions given by

$$
\begin{aligned}
u_{1}(c) & \equiv \frac{c^{1-\gamma}}{1-\gamma}, \\
u_{2}(x, c) & \equiv x_{4} u_{1}(c)=\frac{x_{4} c^{1-\gamma}}{1-\gamma}
\end{aligned}
$$

for some strictly positive constant $\gamma \in \mathbb{N}$ which represents the agents' common relative risk aversion coefficient ${ }^{22}$ and we assume that the aggregate endowment is given by

$$
\bar{g}\left(X_{t}\right) \equiv X_{1 t}
$$

The interpretation of this model is based on the assumption that agents have heterogenous beliefs about the evolution of the economy. The first agent believes that the growth rate of the aggregate endowment process is $X_{2}$ while the second one believes that it is $X_{3}$. The remaining state variable represents the density of the beliefs of agent 2 with respect to those of agent 1 and appears multiplicatively in the definition of the utility function of agent 2 in order to allow us to compute the expected utilities of both agents under the same probability measure.

\footnotetext{
${ }^{22}$ The assumption of an integer coefficient of relative risk aversion is imposed in order to guarantee that the finite horizon price function $\pi$ of equation (16) can be computed in closed form, see Dumas et al. (2008, Section 3) for details.
} 
Since there are two independent sources of risk in the model we know that one riskless asset and at least two risky securities are needed in order to implement the Arrow-Debreu equilibrium by continuous trading. Dumas et al. (2008) consider the case where these two securities are given by an annuity in zero net supply and a claim to the aggregate endowment but they do not actually prove that this menu of securities delivers complete markets. We now fill this gap by showing that in their model dynamic market completeness can be obtained by direct application of the results of this section.

By slightly extending the results of Proposition 2 to allow for state dependent utility functions we obtain that in the above economy equilibrium price of the consumption good takes the form

$$
m\left(t, X_{t}, \rho, \lambda\right)=e^{-\rho t} \mathbf{m}\left(X_{t}, \lambda\right)=e^{-\rho t} \frac{1}{\lambda_{2}}\left(\frac{a+X_{4 t}^{b}}{X_{1 t}}\right)^{\gamma}
$$

where the constant $\lambda_{i} \in(0,1)$ represents the weight of agent $i$ in the definition of the social welfare function and we have set $b \equiv 1 / \gamma, a \equiv\left(\lambda_{2} / \lambda_{1}\right)^{b}>0$. Using this expression in conjunction with the definition of the dividends we obtain that the sum of determinants in equation (20) is explicitly given by

$$
\sum_{i=1}^{2} \operatorname{det} C_{i}(x, \lambda)=\frac{a b(b-1)\left(x_{2}-x_{3}\right)+\sigma_{1}^{2}\left(a+x_{4}^{b}\right)}{\sigma_{1} b\left(a+x_{4}^{b}\right)^{2}} x_{1} x_{4}^{b} \sigma_{32} \text {. }
$$

Since the function on the right hand side of the above expression is real analytic in $x \in \mathcal{X}$ and not identically zero we know that its zero set has measure zero. Furthermore, a detailed inspection of the closed form results of Dumas et al. (2008, Equations (36) and (37)) show that all the conditions of Assumption E are satisfied ${ }^{23}$ and the existence of an equilibrium with dynamically complete markets now follows from Proposition 5.

The above results show that in infinite horizon economies dynamic market completeness can be deduced from the structure of dividends under suitable regularity conditions. A notable difference between finite and infinite horizon economies it that for the latter we do not need to assume any real analyticity. The reason behind this simplification is that, with an infinite horizon and identical rates of time preference, the gradient of the price function is automatically real analytic in $\rho$ as soon as it is well-defined and this is all we need to propagate the non degeneracy of the dividends to the prices. In contrast, in the finite horizon

\footnotetext{
${ }^{23}$ The precise definition of the lower bound on the agents' common rate of time preference can be found in Dumas et al. (2008, Lemma 6), we omit the details
} 
case we have to assume that the price function is real analytic in $(t, x)$ and, as we illustrate in Section 4 below, the conditions needed to guarantee that this assumption holds for a given model can be quite complicated.

\section{Applications}

Given the results of the previous section, proving the existence of an equilibrium with complete markets reduces to finding conditions under which the candidate prices are real analytic for the finite horizon case, and satisfy Assumption E for the infinite horizon case.

In this section we provide conditions under which these properties hold for two concrete classes of models: those where the state variables follow a vector autoregressive process and those where each of the state variable is an autonomous diffusion process. At the end of the section we also discuss whether real analyticity is crucial for market completeness. In particular, we show that as soon as one relaxes this requirement it is possible to construct examples of models which satisfy all the other conditions but nonetheless fail to generate dynamically complete markets.

\subsection{Vector autoregressive processes}

Assume that the vector of state variables evolves according to a continuous-time vector autoregressive process of the form

$$
d X_{t}=\left(b(t)-A(t) X_{t}\right) d t+\sigma_{X}(t) d Z_{t}
$$

where $Z \in \mathbb{R}^{d}$ is a Brownian motion and $b \in \mathbb{R}^{n}, A \in \mathbb{R}^{n \times n}$ and $\sigma_{X} \in \mathbb{R}^{n \times d}$ are real analytic functions of time such that the matrix

$$
\Sigma_{X}(t) \equiv \sigma_{X}(t) \sigma_{X}(t)^{\top}
$$

has rank at least $d$ for all $t \in[0, T]$. This class of models includes as a special case the model studied by Anderson and Raimondo (2008) where the state variables coincide with the underlying Brownian motions but it is significantly more flexible as it allows for arbitrary mean reverting Gaussian processes with deterministic coefficients.

Consider first a finite horizon economy, and let $p(\tau, x, \tau+\theta, y)$ denote the transition density of the state variables from $x$ to $y$ over a time interval of length $\theta$ starting at time $\tau$. With this notation we have that the candidate price function 
defined in equation (9) can be written as a double integral:

$$
\begin{aligned}
m(t-, x) P(t, x)=\int_{\mathbb{R}^{d}} p(t, x, T, y) m(T, y) G(y) d y & \\
& +\int_{t}^{T} \int_{\mathbb{R}^{d}} p(t, x, \tau, y) m(\tau, y) g(\tau, y) d y d \tau .
\end{aligned}
$$

In order to obtain the existence of an equilibrium with dynamically complete markets we need to find conditions under which the above integrals are real analytic as functions of time and the state variables. While real analyticity with respect to the state variables can be obtained under rather weak integrability conditions, real analyticity in time is much more difficult to establish. Using Assumption A in conjunction with the result of Proposition 2 and the fact that $p$ is a Gaussian transition density we have that the integrands are all real analytic in time but this is not sufficient to show that the integrals themselves are real analytic in time. To obtain this property we also need to know that the radius of time analyticity of the integrands is uniformly bounded from below. This is the purpose of the following:

Definition 3: A function $f$ belongs to the class $\mathcal{K}$ if it is real analytic in $(t, x) \in$ $(0, T) \times \mathbb{R}^{n}$ and such that

$$
\sup _{\tau \in \mathcal{O}}|f(\tau, x)| \leq e^{K\|x\|^{\alpha}} K
$$

for some nonnegative constants $K$ and $\alpha<2$ and some complex neighborhood of the time interval $[0, T]$.

THEOREM 4: Consider a finite horizon economy where the state variables evolve according to equation (21). Assume that $n=d$ and that the function

$$
f(t, x) \equiv m(t, x) \bar{g}(t, x)+m(T, x) \bar{G}(x)
$$

belongs to the class $\mathcal{K}$ of Definition 3. Then there exists an equilibrium with dynamically complete markets under either of the following conditions:

(a) Equation (11) holds for almost every $x \in \mathbb{R}^{d}$,

(b) There are no terminal dividends and either of equations (13) and (15) holds for almost every $x \in \mathbb{R}^{d}$.

The above result shows that if the state variables of the economy follow a vector autoregressive process then an upper bound on discounted dividends are sufficient to guarantee the existence of an equilibrium with dynamically complete 
markets. The form of the upper bound is quite intuitive given the decay of the Gaussian transition density associated with the state variables. What is special about this upper bound is that it must hold not only for real values of $t$ but also for complex ones in a neighborhood of the time interval $[0, T]$.

A significant restriction imposed by Theorem 4 is that it requires the number of state variables $(n)$ to equal the number of sources of risk $(d)$. The reason behind this restriction is that, when there are more state variables than sources of risk $(n>d)$, the degeneracy of the instantaneous variance-covariance matrix $\Sigma_{X}(t)$ causes the price integral to blow up for complex values of the time argument. This does not mean, however, that there is no way to analytically continue the candidate price function in the complex domain but we have not been able to do so with the techniques of this paper.

When $b \equiv \mathbf{0}_{d}, A \equiv \mathbf{0}_{d \times d}$ and $\sigma \equiv \mathbb{I}_{d \times d}$ the state variables coincide with the underlying Brownian motions and the model collapses to that of Anderson and Raimondo (2008) albeit with a slightly different market structure. In that case Theorem 4 shows that the conditions imposed by these authors are in general not sufficient for the validity of their main result since they only require that their marginal utility bounds (see their equation (2) p. 851) hold for real values of $t$ and not for complex ones. The only case where their arguments apply without any additional assumption is when dividends are time independent and agents have identical rates of time preference. Indeed, in that case we have that

$$
f(t, x)=e^{-\rho t} n(x) \bar{g}(x)+e^{-\rho T} n(x) \bar{G}(x)
$$

for some nonnegative function $n$ and it follows that the bound automatically hold for complex values of $t$ if they hold for real ones.

In order to verify the upper bound in Theorem 4 it is necessary to impose conditions on the utility functions and the dividends which jointly determine the equilibrium consumption price. This is easily done when intermediate dividends are time independent and agents have identical rates of time preference since in that case it suffices to assume that (22) holds at a single point in time. In the case where time preference rates are heterogenous and/or intermediate dividends are time dependent, sufficient conditions for general utility functions become very technical. For this reason we formulate precise conditions only for the benchmark case of constant relative risk aversion.

Proposition 6: Consider a finite horizon economy where the state variables evolve according to equation (21). Assume that $n=d$ and that

(a) Agent a has subjective rate of time preference $\rho_{a} \geq 0$ and his utility 
functions are given by

$$
u_{a}(c)=v_{a}(c) \equiv \frac{c^{1-\gamma_{a}}-1}{1-\gamma_{a}}
$$

for some strictly positive relative risk aversion coefficient $\gamma_{a}$.

(b) The dividends of stock $i$ are given by

$$
\begin{aligned}
G_{i}(x) & =\nu_{i}(x), \\
g_{i}(t, x) & =e^{\delta_{i} t} \kappa_{i}(x),
\end{aligned}
$$

for some constant $\delta_{i}$ and some real analytic functions $\left(\nu_{i}, \kappa_{i}\right)$ such that

$$
e^{-J_{i}\|x\|^{\alpha}} J_{i} \leq \nu_{i}(x), \kappa_{i}(x) \leq e^{K_{i}\|x\|^{\alpha}} K_{i}
$$

for some strictly positive constants $J_{i}, K_{i}$ and $\alpha<2$.

Then there exists an equilibrium with dynamically complete markets as soon as either of conditions (a) or (b) in Theorem 6 is satisfied.

We now close this section by providing conditions for the existence of an equilibrium with dynamically complete markets in an infinite horizon economy where the state variables follow a vector autoregressive process. In that case the required conditions are much weaker as we no longer need to establish any real analyticity. In particular, we can now treat the case of economies where there are more state variables than there are sources of risk.

In order to accommodate an infinite horizon, and to guarantee that the distribution of the state variables is nondegenerate despite the fact that there can be more state variables than sources of risk we impose the following:

Assumption F: The coefficients $b, A$ and $\sigma_{X}$ are constant and such that

(a) The variance-covariance matrix of the state variables

$$
\Omega(\tau) \equiv E\left[\left(X_{\tau}-E\left[X_{\tau}\right]\right)\left(X_{\tau}-E\left[X_{\tau}\right]\right)^{\top}\right]
$$

is positive definite for all $\tau>0$.

(b) Either all the eigenvalues of $A$ have strictly positive real parts or $A$ is diagonalizable and all its eigenvalues have nonnegative real parts.

TheOrem 5: Consider an infinite horizon economy where the state variables evolve according to equation (21) and suppose that Assumption D and Assumption F hold true. If 
(a) There exists a strictly positive constant $C$ such that

$$
\mathbf{m}(x, \lambda) \bar{g}(x) \leq e^{C\|x\|} C
$$

for all $x \in \mathbb{R}^{n}$,

(b) Either of equations (18) and (20) hold for all $x \in \mathbb{R}^{n}$

then there exists a nonnegative constant $\rho_{0}$ such that the consumption allocation associated with $\lambda \in \mathcal{S}$ gives rise to an equilibrium with dynamically complete markets for all but countably many $\rho>\rho_{0}$.

In order to apply the above results it suffices to find joint conditions on utility functions and dividends under which equation (26) holds. When agents have power utility functions with heterogenous risk aversion coefficients such conditions can be deduced from those of Proposition 6. Indeed, if the time independent dividends satisfy

$$
e^{-J_{i}\|x\|} J_{i} \leq g_{i}(x) \leq e^{K_{i}\|x\|} K_{i}
$$

for some strictly positive constants $J_{i}, K_{i}$ then the arguments used in the proof of Proposition 6 show that equation (26) holds for some strictly positive constant $C$ and it follows that an equilibrium exists for almost all sufficiently large value of the agents' common rate of time preference.

The lower bound $\rho_{0}$ can be computed explicitly in a number of cases. In particular, if all the eigenvalues of the matrix $A$ have strictly positive real parts then the state variables are stationary and it follows that $\rho_{0}=0$. On the other hand, if $A \equiv \mathbf{0}_{n \times n}$ then the state variables are correlated Brownian motions with drift and the lower bound is given by

$$
\rho_{0}=C b^{\top} \mathbf{1}_{d}+\frac{1}{2} C^{2} \mathbf{1}_{d}^{\top} \Sigma_{X} \mathbf{1}_{d}
$$

In the general case where some of the eigenvalues of the matrix $A \neq \mathbf{0}_{n \times n}$ are allowed to have vanishing real parts the nonnegative constant $\rho_{0}$ can still be computed in closed form but its expression is complicated and not very informative so we omit it.

In order to apply the result of Theorem 5 we have to check that the variancecovariance matrix of the state variables over an interval of strictly positive length is non degenerate. Since the state variables are Gaussian processes this matrix can in general be computed explicitly (see for example Karatzas and Shreve 
(1998, Chapter 5.6)) and so checking the validity of Assumption F basically reduces to the computation of a determinant as the following example illustrates.

Example 3: Consider an infinite horizon economy similar to that of Example 1 but driven by three state variables which evolve according to

$$
\begin{aligned}
& d X_{1 t}=\left(X_{3 t}-\left\|\sigma_{1}\right\|^{2} / 2\right) d t+\sigma_{1}^{\top} d Z_{t} \\
& d X_{2 t}=\left(X_{3 t}-\left\|\sigma_{2}\right\|^{2} / 2\right) d t+\sigma_{2}^{\top} d Z_{t} \\
& d X_{3 t}=\kappa\left(\theta-X_{3 t}\right) d t+\sigma_{3}^{\top} d Z_{t}
\end{aligned}
$$

where $Z \in \mathbb{R}^{2}$ is a Brownian motion and $\left(\kappa, \theta, \sigma_{1}, \sigma_{2}, \sigma_{3}\right) \in \mathbb{R} \times \mathbb{R} \times \mathbb{R}^{2} \times \mathbb{R}^{2} \times \mathbb{R}^{2}$ are constants such that $\left\|\sigma_{3}\right\| \neq 0$. The financial market consists in a locally riskless asset and two stocks whose dividends are given by

$$
g_{i}\left(X_{t}\right) \equiv \exp \left(X_{i t}\right), \quad i=1,2 .
$$

This example generalizes the multi-asset models of Cochrane et al. (2008) and Martin (2008) by allowing the growth rates of the individual dividends to be driven by a common, imperfectly correlated, stochastic factor which can be interpreted as a proxy for business cycles in the economy.

As can be seen from equations (27), (28) and (29) the matrix $A$ associated with this example is diagonalizable and has eigenvalues $(0,0, \kappa)$ so we have that part (b) of Assumption F holds as soon as $\kappa$ is nonnegative. On the other hand, standard results on linear Gaussian processes (see Karatzas and Shreve (1998, Chapter 5.6)) show that the determinant of the variance-covariance matrix is given by

$$
\operatorname{det} \Omega(\tau)=F(\tau)\left\|\sigma_{3}\right\|^{2}\left(\operatorname{det}\left(\sigma_{1}, \sigma_{3}\right)-\operatorname{det}\left(\sigma_{2}, \sigma_{3}\right)+\kappa \operatorname{det}\left(\sigma_{1}, \sigma_{2}\right)\right)
$$

for some function $F: \mathbb{R}_{+} \rightarrow \mathbb{R}$ which depends only on $\kappa$ and whose only zero is located at the origin. In particular, part (a) of Assumption $\mathrm{F}$ holds true provided that $\sigma_{2} \neq \sigma_{1}, \operatorname{det}\left(\sigma_{1}, \sigma_{3}\right) \neq 0$ and, since the dividends satisfy condition (a) of Theorem 5 , we have that the existence of a complete markets equilibrium rests entirely on condition (b).

If the instantaneous volatilities of the first two state variables are linearly independent then the volatility matrix of the dividends

$$
\sigma_{g}(x)=\frac{\partial g}{\partial x}(x) \sigma=\left(\begin{array}{c}
e^{x_{1}} \sigma_{1}^{\top} \\
e^{x_{2}} \sigma_{2}^{\top}
\end{array}\right)
$$


is invertible for almost all $x \in \mathbb{R}^{3}$ and the existence of a complete markets equilibrium now follows from the first order condition of Theorem 5. Surprisingly, the condition that the dividend volatilities be linearly independent is sufficient but not necessary for the existence of an equilibrium with complete markets. To see this assume that $\sigma_{2}=\alpha \sigma_{1}$ for some $\alpha$ and recall that, since agents have homogenous power utility functions, the equilibrium consumption price is given by

$$
m(t, x)=e^{-\rho t} A^{\gamma-1}\left(e^{x_{1}}+e^{x_{2}}\right)^{-\gamma} .
$$

A straightforward, albeit tedious, computation shows that the sum of determinants in equation (20) is explicitly given by

$$
\sum_{i=1}^{2} C_{i}(x)=e^{x_{1}+x_{2}}(1-\alpha)(1-\gamma) \operatorname{det}\left(\sigma_{1}, \sigma_{3}\right)
$$

and it follows from the second order condition of Theorem 5 that an equilibrium with dynamically complete markets exists as soon as $\alpha \neq 1, \gamma \neq 1, \operatorname{det}\left(\sigma_{1}, \sigma_{3}\right) \neq$ 0 . Each of these parametric restrictions is necessary for the existence of a complete markets equilibrium. Indeed, if $\alpha=1$ then the stocks pay the same dividends at all times and so markets can never be complete with respect to the underlying filtration. Similarly, if $\operatorname{det}\left(\sigma_{1}, \sigma_{3}\right)=0$ then the three state variables are effectively driven by the one dimensional Brownian motion $\bar{Z}_{t}=\sigma_{1}^{\top} Z_{t} /\left\|\sigma_{1}\right\|$ and so there is no way for markets to be complete with respect to the filtration generated by the two dimensional Brownian motion $Z$. Finally, assume that $\gamma=1$ so that the representative agent has logarithmic utility. In that case it can be shown that the equilibrium stock prices do not depend on the state variable $X_{3}$ which drives the common growth rate of the dividends ${ }^{24}$ and it follows that markets are incomplete since the dividends are perfectly correlated.

\subsection{Autonomous diffusion processes}

For a second application we consider the case where each of the state variables is an autonomous diffusion process. More precisely, we now assume that for each $i$ the associated coordinate of the vector of state variables evolves according to the one dimensional stochastic differential equation

$$
d X_{i t}=\mu_{i}\left(X_{i t}\right) d t+\sigma_{i}\left(X_{i t}\right) d Z_{i t} .
$$

\footnotetext{
${ }^{24}$ This follows from the fact that, since the dividends depend symmetrically on $X_{3}$, the dynamics of the discounted dividends $m(t, x) g_{i}(x)=e^{-\rho t} g_{i}(x) / \bar{g}(x)$ are independent of $X_{3}$.
} 
for some continuously differentiable drift and volatility functions $\mu_{i}, \sigma_{i}$ where $Z_{i}$ is a one dimensional Brownian motion. To simplify the analysis we will further assume that the state space of the $i$-th coordinate is given by $\mathcal{X}_{i} \equiv\left(\ell_{i}, \infty\right)$ for some constant $\ell_{i} \geq-\infty$, and that $X_{i}$ does not reach the boundaries of its spate space in finite time. ${ }^{25}$

Since each coordinate is autonomous and driven by a different Brownian motion we have that the state variables are mutually independent and it follows that the transition density of the vector is given by

$$
p(t, x, y)=\prod_{i=1}^{d} q_{i}\left(t, x_{i}, y_{i}\right), \quad \forall(t, x, y) \in(0, T] \times \mathcal{X}^{2},
$$

where $q_{i}$ is the transition density of the $i$-th coordinate and $\mathcal{X} \equiv \prod_{i=1}^{d} \mathcal{X}_{i}$. The main difference between this model and that of the previous example is that in general we do not have an explicit expression for the transition densities of the various state variables. To circumvent this difficulty we will assume that the unknown transition densities satisfy the following a priori bounds:

Assumption G: For each $i$ there are strictly positive constants $\left(\alpha_{i}, C_{i}\right)$ and locally bounded functions $\left(K_{i}, L_{i}\right)$ such that

$$
q_{i}(t, x, y) \leq K_{i}(x) L_{i}(y) t^{-\alpha_{i}} e^{-C_{i}\left(M_{i}(x)-M_{i}(y)\right)^{2} / t}
$$

for all $(t, x, y) \in[0, T] \times \mathcal{X}_{i}^{2}$ where

$$
M_{i}(x)=\int_{c_{i}}^{x} \frac{d z}{\sigma_{i}(z)}
$$

and $c$ is an arbitrary point in $\mathcal{X}$.

The upper bound in equation (31) implicitly determines the growth condition that the discounted dividends should satisfy for the price integrals to be welldefined but it is not sufficient to guarantee that the price function is real analytic in time. To achieve this we need not only that the integrals be well-defined but also that the integrands be real analytic in time and that their radius of analyticity be uniformly bounded from below.

As far as the transition densities are concerned, these properties follow from Assumption G since it can be shown that if equation (31) holds for real values of

\footnotetext{
${ }^{25}$ The assumption that $r_{i} \equiv \sup \left\{x \in \mathbb{R}: x \in \mathcal{X}_{i}\right\}=\infty$ can be relaxed but it is in general harmless as most of the models in the literature use state variables whose coordinates take values in either the positive real line or the whole real line.
} 
$t$ then it automatically holds for complex ones, see Davies (1997, Lemma 2) and Appendix D.2. The situation with the discounted dividends is more complicated since there is no guarantee that the bound holds for complex values of $t$ if it holds for real ones. As in the previous section, we will circumvent this difficulty by imposing an upper bound in a complex neighborhood of $[0, T]$.

Definition 4: A function $f:[0, T] \times \mathcal{X} \rightarrow \mathbb{R}$ belongs to the class $\mathcal{M}$ if it is real analytic in $(t, x) \in(0, T) \times \mathcal{X}$ and such that

$$
\int \sup _{\tau \in \mathcal{O}}|f(\tau, y)| \prod_{i=1}^{d} L_{i}\left(y_{i}\right) e^{-\varepsilon M_{i}\left(y_{i}\right)^{2}} d y<\infty
$$

for any $\varepsilon>0$ and some complex neighborhood $\mathcal{O} \supseteq[0, T]$.

THEOREM 6: Consider a finite horizon economy such that Assumption G holds and assume that the function

$$
f(t, x) \equiv m(t, x) \bar{g}(t, x)+m(T, x) \bar{G}(x)
$$

belongs to the class $\mathcal{M}$. Then there exists an equilibrium with dynamically complete markets if either of the following conditions holds:

(a) Equation (11) holds for almost every $x \in \mathbb{R}^{d}$ and $G \in C^{2}(\mathcal{X})$,

(b) There are no terminal dividends and either of equations (13) and (15) holds for almost every $x \in \mathbb{R}^{d}$.

The conditions of Assumption G and Theorem 6 might seem very technical but they are in fact quite easy to verify for concrete models and can be shown to hold for most of the diffusions processes that are routinely used in the asset pricing literature. In particular, the following examples show that these conditions hold for Cox-Ingersol-Ross (CIR) processes and for a wide class of constant elasticity of variance $(\mathrm{CEV})$ processes.

EXAmple 4 (CIR processes): Assume that each of the $d$ state variables evolves according to the square root dynamics

$$
d X_{i t}=\left(a_{i}-b_{i} X_{i t}\right) d t+\sigma_{i} \sqrt{\left|X_{i t}\right|} d Z_{i t}
$$

for some constants $a_{i}>0, b_{i}$ and $\sigma_{i}$ such that $\nu_{i} \equiv\left(2 a_{i} / \sigma_{i}^{2}\right)-1>0$. This last condition guarantees that the unique solution to equation (33) never reaches zero and can be relaxed at the costs of increased notational burden. 
The following result shows that Assumption $G$ is satisfied and gives a simple criterion for a given function to belong the class $\mathcal{M}$ which appears in the statement of Theorem 6 .

Lemma 1: Assumption G holds and a sufficient condition for a real analytic function to belong to the class $\mathcal{M}$ is that

$$
\sup _{\tau \in \mathcal{O}}|f(\tau, x)| \leq e^{K\|x\|^{\delta}} K
$$

for some strictly positive constants $K$ and $\delta<1$ and some complex neighborhood of the time interval $[0, T]$.

In order to obtain the existence of an equilibrium with complete markets for this economy we need to find conditions on the dividends, utility functions and time preference rates under which the function of equation (32) satisfies the bound of Lemma 1 in some complex neighborhood of $[0, T]$. As explained in Section 4.1 this is easily done if agents have identical time preference rates and dividends are time independent since in that case the function depends exponentially on time. In the general case where agents have heterogenous time preference rates and/or dividends are time dependent the situation is more complicated. Nevertheless, we have a simple criterion for finite horizon economies where agents have power utility functions with heterogenous risk aversions and time preference rates.

Proposition 7: Consider a finite horizon economy where the state variables evolve according to equation (33) and assume that

(a) Agent a has subjective rate of time preference $\rho_{a} \geq 0$ and his utility functions are given by

$$
u_{a}(c)=v_{a}(c) \equiv \frac{c^{1-\gamma_{a}}-1}{1-\gamma_{a}}
$$

for some strictly positive relative risk aversion coefficient $\gamma_{a}$.

(b) The dividends of stock $i$ are given by equations (24) and (25) for some constant $\delta_{i} \in \mathbb{R}$ and some functions $\left(\nu_{i}, \kappa_{i}\right)$ such that

$$
e^{-J_{i}\|x\|^{\delta}} J_{i} \leq \nu_{i}(x), \kappa_{i}(x) \leq e^{K_{i}\|x\|^{\delta}} K_{i}
$$

for some strictly positive constants $J_{i}, K_{i}$ and $\delta<1$.

Then there exists an equilibrium with dynamically complete markets as soon as either of conditions (a) or (b) in Theorem 6 is satisfied. 
For a second example of application of the result of Theorem 6 we now turn to constant elasticity of variance $(\mathrm{CEV})$ processes.

ExAmple 5 (CEV processes): Assume that each of the $d$ state variables of the model evolves according to the constant elasticity of variance dynamics

$$
d X_{i t}=\mu_{i} X_{i t} d t+\xi_{i}\left|X_{i t}\right|^{1+\beta_{i}} d Z_{i t}
$$

for some constants $\mu_{i}, \xi_{i}$ and $\beta_{i}>0$. This last condition guarantees that the unique solution to the above equation never reaches the origin and hence is never absorbed.

In order to show that Assumption $G$ is satisfied in this example consider the nonnegative process defined by

$$
Y_{i t} \equiv\left(\xi_{i} \beta_{i} X_{i t}^{\beta_{i}}\right)^{-2}
$$

Applying Itô's lemma to the right hand side and using equation (34) shows that this process evolves according to the square root dynamics of equation (33) with the constants $a_{i}=2+1 / \beta_{i}, b_{i}=2 \mu_{i} \beta_{i}$ and $\sigma_{i}=-2$. Combining this simple observation with the arguments used in the proof of Lemma 1 gives:

Lemma 2: Assumption $\mathrm{G}$ holds and a sufficient condition for a real analytic function to belong to the class $\mathcal{M}$ is that

$$
\sup _{\tau \in \mathcal{O}}|f(\tau, x)| \leq 1+K\|x\|^{\alpha}
$$

for some strictly positive constants $K$ and $\alpha<\bar{\alpha} \equiv 2\left(1+\min _{i} \beta_{i}\right)$ and some complex neighborhood of the time interval $[0, T]$.

In order to obtain the existence of a complete markets equilibrium we need to find conditions on the dividends, utility functions and time preference rates under which the function $f$ of equation (32) satisfies the bound of Lemma 2 in some complex neighborhood of the interval $[0, T]$. As in the previous examples, there are basically two cases where such conditions can be easily formulated: the case of homogenous time preference rates and time independent dividends, and the case of power utility agents with heterogenous time preference rates and risk aversion coefficients. For the latter we have to following analog to Propositions 6 and 7

Proposition 8: Consider a finite horizon economy where the state variables evolve according to equation (34) and assume that 
(a) Agent a has subjective rate of time preference $\rho_{a} \geq 0$ and his utility functions are given by

$$
u_{a}(c)=v_{a}(c) \equiv \frac{c^{1-\gamma_{a}}-1}{1-\gamma_{a}}
$$

for some strictly positive relative risk aversion coefficient $\gamma_{a}$.

(b) The dividends of stock $i$ are given by equations (24) and (25) for some constant $\delta_{i} \in \mathbb{R}$ and some functions $\left(\nu_{i}, \kappa_{i}\right)$ such that

$$
J_{i}\|x\|^{-\alpha} \leq \nu_{i}(x), \kappa_{i}(x) \leq K_{i}\|x\|^{\alpha}
$$

for some strictly positive constants $J_{i}, K_{i}$ and $\alpha<\bar{\alpha}$.

Then there exists an equilibrium with dynamically complete markets as soon as either of conditions (a) or (b) in Theorem 6 is satisfied.

\subsection{Counterexamples}

The three previous examples clearly illustrate that the most difficult part in applying the result of Section 3.2.1 consists in verifying that the candidate price function is real analytic in time. Given this observation it is natural to wonder whether this somehow technical condition is absolutely necessary if one wants to deduce market completeness from the structure of dividends. We show in this section that this is indeed the case by presenting counterexamples which show that, unless this condition is verified, the candidate prices may fail to generate dynamically complete markets even though all the other conditions of Theorem 1 are satisfied. In order to highlight the intuition behind our construction we start with an explicit example of a single stock economy before we move on to economies with multiple stocks.

\subsubsection{A single stock economy}

Assume that the uncertainty is generated by a one dimensional Brownian motion and consider a finite horizon economy populated by a representative agent with initial portfolio $\eta=1$, time preference rate $\rho \geq 0$ and logarithmic utility.

Since there is a representative agent we know that this economy admits a unique equilibrium independently of whether markets are complete or not. Furthermore, exploiting the assumption of logarithmic utility we obtain that in 
this equilibrium the stock price is

$$
S_{t}=1_{\{t<T\}} P\left(t, X_{t}\right)+1_{\{t=T\}} G\left(X_{T}\right)
$$

where the pre-horizon price function is defined by

$$
P\left(t, X_{t}\right)=g\left(t, X_{t}\right)\left((1 / \rho)+e^{-\rho(T-t)}(1-1 / \rho)\right) .
$$

This expression shows that in this example dynamic market completeness is entirely determined by the intermediate dividends. In particular, if we assume that there exists a set of strictly positive measure $\mathcal{R} \subseteq(0, T)$ such that

$$
\frac{\partial g}{\partial x}(t, x)=0
$$

for almost every $(t, x) \in \mathcal{R} \times \mathcal{X}$ then the stock volatility vanishes on $\mathcal{R}$ and it follows that markets are incomplete in equilibrium even though the effective terminal dividend

$$
\mathcal{G}(x)=P(T, x)=g(T, x)
$$

may be chosen in such a way as to satisfy the nondegeneracy condition of Theorem $1 .{ }^{26}$ The reason why the result of Theorem 1 does not apply here is that if equation (37) holds on a set of strictly positive measure then the equilibrium price function fails to be real analytic in time over the whole interval $(0, T)$ and so we cannot propagate the nondegeneracy of the stock volatility from a neighborhood of the terminal time to the whole interval.

\subsubsection{Multiple stocks economies}

Consider a finite horizon economy where the uncertainty is generated by a $d$-dimensional Brownian motion, and assume that the economy is populated by a single agent with time preference rate $\rho \geq 0$ and utility function $u$.

Let further $(g, G)$ denote the dividends of the stocks and assume that the dividends of the first stock are uniformly bounded above and away from zero. The following proposition will allow us to extend the construction of the previous example to economies with multiple risky securities.

\footnotetext{
${ }^{26}$ A simple example of a smooth function which satisfies the condition of Theorem 1 as well as equation (37) on a set of strictly positive measure is $\Gamma(t, x)=\alpha+\beta \exp \left(-x^{2} /(t-\gamma T)^{+}\right)$ for some strictly positive constants $\alpha, \beta$ and $\gamma<1$. In that case the set over which the price function fails to be real analytic in time is simply given by $\mathcal{R}=(0, \gamma T]$.
} 
Proposition 9: Consider an economy as above and let $F_{1} \in C^{1,2}((0, T) \times \mathcal{X})$ be a nonnegative function. Then there exist nonnegative intermediate dividend functions $g_{2}, \ldots, g_{d}$ and a nonnegative constant $K$ such that the equilibrium price of the first stock satisfies $S_{1 t}=F_{1}\left(t, X_{t}\right)+K$ for all $t \in[0, T)$.

In the equilibrium which is described by the above proposition, dynamic market completeness depends to a large extend on the choice of the exogenous function $F_{1}$. In particular, let $\mathcal{R} \subseteq[0, T]$ be a open set of strictly positive measure and assume that we choose the function $F_{1}$ in such a way that

$$
\frac{\partial F_{1}}{\partial x}(t, x)=\mathbf{0}_{d}
$$

for almost every $(t, x) \in \mathcal{R} \times \mathcal{X}$. In that case, the volatility matrix of the equilibrium stock price process is automatically degenerate on the set $\mathcal{R}$ and it follows that markets are incomplete even though the "effective" terminal dividends,

$$
\mathcal{G}(x)=\lim _{t \rightarrow T} P(t, x)=\left(F_{1}(T, x)+K\right) \frac{G(x)}{G_{1}(x)},
$$

can be chosen in such a way as to satisfy the nondegeneracy condition of Theorem 1. As in the previous example, the reason why the result of Theorem 1 does not apply here is that, given our choice for the function $F_{1}$, the equilibrium price function fails to be real analytic over the whole time interval. This clearly shows that real analyticity cannot be dispensed with if one is to deduce market completeness from the properties of the dividends.

REMARK 1: The result of Proposition 9 bears some close connection with the literature on so-called viable diffusion price processes, see Bick (1993), He and Leland (1993) and Wang (1993) among others. In particular, it complements this literature by showing that in an economy with multiple stocks fixing the dividends of one stock does not impose any constraint on its equilibrium price except for a lower bound.

\section{Conclusion}

This paper provides sufficient condition for the existence of an equilibrium with endogenously complete markets in a continuous-time, heterogenous agents economy driven by an arbitrary diffusion process. In contrast to Anderson and Raimondo (2008), our formulation does not require the traded securities to pay terminal dividends. As a result, the horizon of the economy can be either finite 
or infinite and we can include instantaneously risk free bonds in the menu of traded assets as is customary in continuous-time asset pricing. Furthermore, our formulation makes a distinction between state variables and sources of risk and thus allows for a much richer set of dynamics.

Our main results show that if the candidate prices are real analytic as functions of time then market completeness can be deduced from the properties of the dividends. More precisely, we prove that a sufficient condition for market completeness is that the volatility matrix of the dividends be nondegenerate. This intuitive condition is sufficient for endogenous completeness but it is far from being necessary. In particular, if some of the traded assets are fixed income securities then this condition fails but markets may nonetheless be complete. We show how higher order conditions can be derived to deal with such cases and provide an explicit example of an economy with endogenously complete markets where one of the securities is an annuity.

In order to apply our main results it is necessary to prove that the candidate prices are real analytic functions of time and the state variables of the model. While we show that this can be done in some benchmark cases, the proof is in general quite difficult. It is therefore natural to wonder whether real analyticity is absolutely crucial if one is to deduce market completeness from the structure of dividends. We show that this is indeed the case by constructing examples of economies where the dividends have a nondegenerate volatility and markets nonetheless fail to be dynamically complete because the candidate prices are not real analytic.

\section{References}

R. Anderson and R. Raimondo. Equilibrium in continuous-time financial markets: Endogenously dynamically complete markets. Econometrica, 76(4):841-907, 2008.

R. Bansal. Long run risks and risk compensation in equity markets. Chapter V of the Handbook of the Equity Risk Premium edited by Rajnish Mehra, North Holland, 2009.

S. Basak. A general equilibrium model of portfolio insurance. Review of Financial Studies, 8:1059-1090, 1995.

L. Benzoni, P. Collin Dufresne, and R. Goldstein. Can standard preferences explain the prices of out-of-the-money s\&p 500 put options? Working paper, University of California, Berkeley, 2006.

H. Bhamra, L.-A. Kuhn, and I. Strebulaev. The aggregate dynamics of capital structure and macroeconomic risk. Working paper, Stanford University, 2008.

A. Bick. On viable diffusion price processes of the market portfolio. Journal of Finance, 45(2):673-689, 1993. 
Y. Chan and L. Kogan. Catching up with the joneses: Heterogenous preferences and the dynamics of asset prices. Journal of Political Economy, 110(6):1255-1285, 2002.

H. Chang, W. He, and N. Pradhu. The analytic domain in the implicit function theorem. Journal of inequalities in pure and applied mathematics, 4(1):Article 12, 2003.

J. Cochrane, F. Longstaff, and P. Santa Clara. Two trees. Review of Financial Studies, 21(1):347-385, 2008.

G. Constantinides. Intertemporal asset pricing with heterogenous consumers and without demand aggregation. Journal of Business, 55(2):253-267, 1982.

J. Cox, J. Ingersoll, and S. Ross. A theory of the term structure of interest rates. Econometrica, 53(2):385-407, 1985.

B. Davies. Non gaussian aspects of heat kernel behavior. Journal of the London Mathematical Society, 55(2):105-125, 1997.

J. Detemple and A. Serrat. Dynamic equilibrium with liquidity constraints. Review of Financial Studies, 16(2):597-629, 2003.

A. Downes. Bounds for the transition density of time homegeneous diffusion processes. Statistics and Probability Letters, 79:835-841, 2009.

D. Duffie. Stochastic equilibria: existence, spanning number and the "no expected financial gain from trade" hypothesis. Econometrica, 54(5):1161-1183, 1986.

D. Duffie. Dynamic Asset Pricing Theory. Princeton University Press, third edition, 2001.

D. Duffie and C.-F. Huang. Implementing Arrow-Debreu equilibria by continuous trading of few long-lived securities. Econometrica, 53:1337-1356, 1985.

D. Duffie and W. Zame. The consumption-based capital asset pricing model. Econometrica, 57(6):1279-1297, 1989.

B. Dumas. Two person dynamic equilibrium in the capital market. Review of Financial Studies, 2(2):157-188, 1989.

B. Dumas, A. Kurshev, and R. Uppal. Equilibrium portfolio strategies in the presence of sentiment risk and excess volatility. Forthcoming in the Journal of Finance, 2008.

S. Eidelman. Parabolic Systems. Wolters-Noordhoff, Groningen, 1969.

W. Feller. Two singular diffusion problems. Annals of Mathematics, 54(1):173-182, July 1951.

H. He and H. Leland. On equilibrium asset price processes. Review of Financial Studies, 6(3):593-617, 1993.

C.-F. Huang. An intertemporal general equilibrium asset pricing model: The case of diffusion information. Econometrica, 55:117-142, 1987.

J. Hugonnier. Bubbles and multiplicity of equilibria under portfolio constraints. Working paper, University of Lausanne, 2008.

C. M. Joshi and S. Bissu. Some inequalities of Bessel and modified Bessel functions. Journal of the Australian Mathematical Society, Series A: Pure Mathematics and Statistics, 50(2):333-342, 1991.

I. Karatzas and S. Shreve. Brownian Motion and Stochastic Calculus. Springer Verlag, second edition, 1998.

I. Karatzas and S. Shreve. Methods of Mathematical Finance. Springer Verlag, second 
edition, 2001.

I. Karatzas, J. Lehoczky, and S. Shreve. Existence and uniqueness of multiagent equilibrium in a stochastic, dynamic consumption-investment model. Operations Research, 15:80-128, 1990.

I. Karatzas, J. Lehoczky, and S. Shreve. Equilibrium models with singular asset prices. Mathematical Finance, 1:11-30, 1991.

S. Krantz and H. Parks. A primer of real analytic functions. Birkhäuser, Basel, Second edition, 2002.

D. Kreps. Multiperiod securities and the efficient allocation of risk: A comment on the black-scholes option pricing model. In The Economics of Uncertainty and Information, edited by J. McCall, University of Chicago Press, 1982.

F. Longstaff and J. Wang. Asset pricing and the credit market. Working paper, Massachusetts Institute of Technology, 2008.

A. Lunardi. Analytic semigroups and optimal regularity in parabolic problems. Birkhauser, Basel, 1995.

S. Malamud. Long run forward rates and long yields of bonds and options in heterogeneous equilibria. Finance and Stochastics, 12:245-264, 2008.

S. Malamud and E. Trubowitz. Rational factor analysis. Working paper, ETH Zürich, 2006.

I. Martin. The Lucas orchard. Working paper, Stanford University, 2008.

A. Mas Colell and W. Zame. Equilibrium theory in inifnite dimensional spaces. In volume IV of the Handbook of Mathematical Economics, edited by W. Hildenbrand and H. Sonnenscheim, North Holland, 1991.

D. Nualart. The Malliavin calculus and related topics. Springer Verlag, second edition, 2006.

B. Oksendal. Stochastic Differential Equations: An Introduction with Applications. Springer Verlag, Sixth edition, 2007.

R. Radner. Existence of equilibrium of plans, prices and prices expectations in a sequence of markets. Econometrica, 40:289-303, 1972.

T. Santos and P. Veronesi. Labor income and predictable stock returns. Review of Financial Studies, 19:1-44, 2006.

B. Shabat. Introduction to complex analysis. Part II: Functions of several variables, volume 110 of Translations of Mathematical Monographs. American Mathematical Society, 1992.

N. Shikamura. Partial Differential Operators of Elliptic Type. American Mathematical Society, 1992.

J. Siciak. Separately real analytic functions and envelopes of holomorphy of some lower dimensional subsets of $\mathbb{C}^{n}$. Annales Polonici Mathematici, 22:145-171, 1969.

D. Stroock and S. Varadhan. Multidimensional diffusion processes. Springer Verlag, 1979.

O. Vasicek. An equilibrium characterization of the term structure. Journal of Financial Economics, 5:177-188, 1977.

J. Wang. The term structure of interest rates in a pure exchange economy with 
heterogenous investors. Journal of Financial Economics, 41(1):75-110, 1996.

S. Wang. The integrability problem of asset prices. Journal of Economic Theory, 59(1): 199-213, 1993. 


\section{A Technical results}

This appendix gathers some technical results that are needed for the proofs of our main results.

Lemma A.1: If $F: \mathbb{R}_{+} \times \mathcal{X} \rightarrow \mathbb{R}$ is real analytic in $(t, x) \in \mathbb{R}_{+} \times \mathcal{X}$ then

$$
I(t, x)=\operatorname{det} \frac{\partial F}{\partial x}(t, x) \sigma_{X}(t, x)
$$

is also real analytic in $(t, x) \in \mathbb{R}_{+} \times \mathcal{X}$.

Proof. This follows from Krantz and Parks (2002, Proposition 2.2.3) and the fact the determinant of a matrix is a multilinear function of its entries.

QED.

Lemma A.2: Assume that $F: \mathbb{R}_{+} \times \mathcal{X} \rightarrow \mathbb{R}$ is continuous and real analytic in $t \in \mathbb{R}_{+}$ and $F(\cdot, x) \not \equiv 0$ for Lebesque-almost every $x \in \mathcal{X}$. Then there exists a countable set $\mathcal{O} \subset \mathbb{R}_{+}$such that

$$
F(t, x) \neq 0
$$

for each fixed $t \in \mathbb{R}_{+} \backslash \mathcal{O}$ and almost every $x \in \mathcal{X}$.

Proof. Suppose to the contrary that there exists a bounded uncountable ${ }^{27}$ set $\mathcal{O}$ and a family $\left\{A_{t}: t \in \mathcal{O}\right\}$ of Lebesque-measurable subsets of $\mathcal{X}$ of positive measure such that

$$
\left\{(t, x): x \in A_{t}\right\} \subseteq \mathcal{Z}_{F} \equiv\left\{(t, x) \in \mathbb{R}_{+} \times \mathcal{X}: F(t, x)=0\right\}
$$

for each fixed $t \in \mathcal{O}$. By application of Lemma A.3 below this implies that there exists a countably infinite sequence $\left\{t_{k}\right\}_{k=1}^{\infty} \subseteq \mathcal{O}$ such that the set $A_{\infty}=\cap_{k=1}^{\infty} A_{y_{k}}$ has strictly positive Lebesgue measure. By construction, $F\left(t_{k}, x\right)=0$ for all $k$ and all $x \in A_{\infty}$. By the uniqueness theorem for analytic functions of one variable, $F(t, x) \equiv 0$ for any $x \in A_{\infty}$ which is a contradiction.

QED.

Lemma A.3: Let $\Sigma \subseteq \mathbb{R}$ be a $n$ uncountable set and $\left\{A_{\sigma}: \sigma \in \Sigma\right\}$ denote a family of Lebesque-measurable subsets of $\mathcal{X}$ such that $A_{\sigma}$ has strictly positive Lebesgue measure for every $\sigma \in \Sigma$. Then there exists a countably infinite set $\Phi \subseteq \Sigma$ such that $A^{*} \equiv \cap_{\sigma \in \Phi} A_{\sigma}$ has strictly positive Lebesgue measure.

Proof. Let $\nu$ denote Lebesgue measure on $\mathbb{R}^{n}$. Since $\left\{1_{A_{\sigma}}: \sigma \in \Sigma\right\}$ is a non empty subset of $L^{1}(\mathcal{X}, \nu)$ it follows from well-known results in functional analysis that there exists a non trivial subset $A^{c}$ of $\mathcal{X}$ and a sequence $\left\{A_{\sigma_{k}}\right\}_{k=1}^{\infty}$ of elements of $\left\{A_{\sigma}: \sigma \in \Sigma\right\}$ such that

$$
\lim _{k \rightarrow \infty} \int_{\mathcal{X}}\left|1_{A_{\sigma_{k}}}(x)-1_{A^{c}}(x)\right| d x=\lim _{k \rightarrow \infty} \nu\left(A_{\sigma_{k}} \triangle A^{c}\right)=0
$$

where $\triangle$ denotes the set theoretic symmetric difference. Let now $\Phi=\left\{\phi_{n}\right\}_{n=1}^{\infty}$ be a subsequence of $\left\{\sigma_{k}\right\}_{k=1}^{\infty}$ such that

$$
\nu\left(A_{\phi_{n}} \triangle A^{c}\right) \leq 2^{-(n+1)} \nu\left(A^{c}\right)
$$

\footnotetext{
${ }^{27}$ If $\mathcal{O}$ is unbounded and uncountable then there exist $n \in \mathbb{N}$ such that $\mathcal{O} \cap[-n, n]$ is uncountable (since otherwise $\mathcal{O}$ itself would be countable) and we may simply replace $\mathcal{O}$ by $\mathcal{O} \cap[-n, n]$.
} 
for all $n \geq 1$ and set $A^{*} \equiv \cap_{\sigma \in \Phi} A_{\sigma}=\cap_{n=1}^{\infty} A_{\phi_{n}}$. With this notation

$$
\begin{aligned}
\nu\left(A^{*}\right) & \geq \nu\left(A^{*} \cap A^{c}\right) \\
& \geq \nu\left(A^{c}\right)-\nu\left(A^{*} \triangle A^{c}\right) \\
& \geq \nu\left(A^{c}\right)-\sum_{n=1}^{\infty} \nu\left(A_{\phi_{n}} \triangle A^{c}\right) \\
& \geq \nu\left(A^{c}\right)-\sum_{n=1}^{\infty} 2^{-(n+1)} \nu\left(A^{c}\right)=\frac{\nu\left(A^{c}\right)}{2}
\end{aligned}
$$

and the desired result now follows from the fact that the set $A^{c}$ has strictly positive measure by construction.

QED.

The following result guarantees that the solution to a parabolic partial differential equation with real analytic coefficients is real analytic in the space variable and will be instrumental in showing that the candidate prices are real analytic as functions of the state variables.

Theorem A.1: Assume that the functions $d_{i}, f, V_{i j}$ are real analytic in $(t, x)$ for all $(i, j)$ and that $V(t, x) \in \mathbb{R}^{n \times n}$ is positive definite and nondegenerate for any $(t, x)$. Then any solution to the partial differential equation

$$
-\frac{\partial F}{\partial t}=f(t, x)+\sum_{i=1}^{n} d_{i}(t, x) \frac{\partial F}{\partial x_{i}}+\sum_{i, j=1}^{n} V_{i j}(t, x) \frac{\partial^{2} F}{\partial x_{i} \partial x_{j}}
$$

is real analytic in $x$ and its analyticity radius is uniformly bounded from below when $t$ varies in a compact set.

Proof. See Eidelman (1969, Theorem 6.2, p. 221)

QED.

The final result in this section gives conditions under which a separately real analytic function is jointly real analytic and will be necessary to establish the real analyticity of the candidate price function in the applications of Section 4

Theorem A.2: Assume that $F(t, x)$ is real analytic in $t \in(0, T)$ for each fixed $x \in \mathcal{X}$ and real analytic in $x \in \mathcal{X}$ for each fixed $t \in(0, T)$. If

(a) The radius of time analyticity is uniformly bounded from below when $x$ varies on compact subsets of $\mathcal{X}$,

(b) The radius of space analyticity is uniformly bounded from below when t varies on compact subsets of $(0, T)$,

then $F$ is jointly real analytic in $(t, x) \in(0, T) \times \mathcal{X}$.

Proof. This follows from the results of Siciak (1969).

QED.

\section{B Proofs for Section 2.5}

Proof of Proposition 1. The result follows from Malamud (2008, Theorem 3.1) and Malamud and Trubowitz (2006, Theorem 3.1) after some straightforward modifications. We omit the details.

QED.

Proof of Proposition 2. The result follows from Huang (1987, Propositions 3.1 and 3.2) and the analytic implicit function theorem (see Krantz and Parks (2002, Section $2.3)$ ). We omit the details.

QED. 


\section{Proofs for Section 3: Endogenous completeness}

\section{C.1 Candidate price functions}

Proof of Proposition 3. The proposition follows from standard continuous-time asset pricing results, see for example Duffie (2001, Theorem 6.I).

QED.

\section{C.2 Finite horizon economies}

Proof of TheOREM 1. By definition of the candidate price function we have

$$
\lim _{t \rightarrow T} P(t, x)=\mathcal{G}(x) .
$$

Combining this with the assumption that $P \in C^{0,1}((0, T] \times \mathcal{V})$ then gives

$$
\lim _{t \rightarrow T} \sigma_{P}(t, x)=\lim _{t \rightarrow T} \frac{\partial P}{\partial x}(t, x) \sigma_{X}(t, x)=\sigma_{\mathcal{G}}(T, x)
$$

and therefore

$$
I(t, x) \equiv \operatorname{det} \sigma_{P}(t, x)=\operatorname{det} \sigma_{\mathcal{G}}(T, x)+o(1)
$$

for all $x \in \mathcal{V}$. Together with the above expansion the assumption of the statement implies that $I$ is not identically zero. On the other hand, since $P$ is real analytic in $(t, x) \in(0, T) \times \mathcal{X}$ we know from Lemma A.1 that $I$ also is real analytic in $(t, x) \in$ $(0, T) \times \mathcal{X}$. Combining these properties with the result of Lemma A.2 shows that we have $I(t, x) \neq 0$ for almost every $(t, x) \in(0, T) \times \mathcal{X}$ and the desired result now follows from Proposition 3.

QED.

Proof of TheOrem 2. The assumptions of the statement and the definition of the candidate prices jointly imply that the function $P$ is a classical solution to the partial differential equation given by

$$
-\frac{\partial P}{\partial t}(t, x)=g(t, x)+\frac{\mathcal{L}(m P)(t, x)}{m(t, x)}+P(t, x) \frac{\partial \log m}{\partial t}(t, x)
$$

for all $(t, x) \in(0, T) \times \mathcal{X}$ and has terminal value equal to zero.

Using the smoothness of the coefficients in conjunction with Proposition 2 and the assumption that $P \in C^{2}((0, T] \times \mathcal{X})$ we obtain

$$
\lim _{t \rightarrow T} P(t, x) \frac{\partial \log m}{\partial t}(t, x)=\lim _{t \rightarrow T} \frac{\mathcal{L}(m P)(t, x)}{m(t, x)}=\mathbf{0}_{d}
$$

and it now follows from equation (38) that

$$
\lim _{t \rightarrow T} \frac{\partial P}{\partial t}(t, x)=-g(T, x) .
$$

Since $P \in C^{2}((0, T] \times \mathcal{X})$ by assumption this further implies that

$$
\begin{aligned}
\lim _{t \rightarrow T} \frac{\partial \sigma_{P}}{\partial t}(t, x) & =\lim _{t \rightarrow T}\left(\frac{\partial^{2} P}{\partial t \partial x} \sigma_{X}+\frac{\partial P}{\partial x} \frac{\partial \sigma_{X}}{\partial t}\right)(t, x) \\
& =\lim _{t \rightarrow T} \frac{\partial^{2} P}{\partial t \partial x}(t, x) \sigma_{X}(t, x)=-\sigma_{g}(T, x)
\end{aligned}
$$


and therefore

$$
\sigma_{P}(t, x)=(T-t) \sigma_{g}(T, x)+o(T-t)
$$

where we have used the fact the candidate price function converges to zero at the terminal time. Combining the above expansion with an argument similar to that used in the proof of Theorem 1 then shows that

$$
I(t, x) \equiv \operatorname{det} \sigma_{P}(t, x) \neq 0
$$

for almost every $(t, x) \in(0, T) \times \mathcal{X}$ as soon as the intermediate dividends satisfy equation (13) and the desired result now follows from Proposition 3.

QED.

Proof of Proposition 4. Since $P \in C^{3}((0, T] \times \mathcal{X})$ we have that the candidate price function satisfies equation (38) and differentiating with respect to time on both sides of the partial differential equation we obtain

$$
\begin{aligned}
-\frac{\partial^{2} P}{\partial t^{2}}(t, x)=\frac{\partial g}{\partial t}(t, x) & +\frac{\partial}{\partial t} \frac{\mathcal{L}(m P)(t, x)}{m(t, x)} \\
& +\frac{\partial P}{\partial t}(t, x) \frac{\partial \log m}{\partial t}(t, x)+P(t, x) \frac{\partial^{2} \log m}{\partial t^{2}}(t, x) .
\end{aligned}
$$

Using the assumed regularity of the coefficients in conjunction with Proposition 2, equation (39) and the assumption that $P \in C^{3}((0, T] \times \mathcal{X})$ we obtain

$$
\lim _{t \rightarrow T} \frac{\partial}{\partial t} \frac{\mathcal{L}(m P)(t, x)}{m(t, x)}=-\frac{\mathcal{L}(m g)(T, x)}{m(T, x)} .
$$

On the other hand, it follows from equation (39) and the continuity of the candidate price function that we have

$$
\lim _{t \rightarrow T}\left(\frac{\partial P}{\partial t} \frac{\partial \log m}{\partial t}+P \frac{\partial^{2} \log m}{\partial t^{2}}\right)(t, x)=-g(T, x) \frac{\partial \log m}{\partial t}(T, x),
$$

and combining this with equations (40) and (41) we conclude that

$$
\begin{aligned}
\lim _{t \rightarrow T} \frac{\partial^{2} P}{\partial t^{2}}(t, x) & =-\frac{\partial g}{\partial t}(T, x)+\frac{\mathcal{L}(m g)(T, x)}{m(T, x)}+g(T, x) \frac{\partial \log m}{\partial t}(T, x) \\
& =\frac{\mathcal{D}(m g)(T, x)}{m(T, x)}-2 \frac{\partial g}{\partial t}(T, x) \equiv \bar{H}(x) .
\end{aligned}
$$

Since $P \in C^{3}((0, T] \times \mathcal{X})$ by assumption this further implies that

$$
\begin{aligned}
\lim _{t \rightarrow T} \frac{\partial^{2} \sigma_{P}}{\partial t^{2}}(t, x) & =\lim _{t \rightarrow T}\left(\frac{\partial^{3} P}{\partial t^{2} \partial x} \sigma_{X}+2 \frac{\partial^{2} P}{\partial t \partial x} \frac{\partial \sigma_{X}}{\partial t}+\frac{\partial P}{\partial x} \frac{\partial \sigma_{X}}{\partial t}\right)(t, x) \\
& =\frac{\partial \bar{H}}{\partial x}(x) \sigma_{X}(T, x)-2 \frac{\partial g}{\partial x}(T, x) \frac{\partial \sigma_{X}}{\partial t}(T, x)=H(x)
\end{aligned}
$$

and therefore

$$
\Phi(t, x) \equiv \sigma_{P}(t, x)-(T-t) \sigma_{g}(T, x)-\frac{1}{2}(T-t)^{2} H(x)=o(T-t)^{2}
$$

where we have used the fact the candidate price function converges to zero at the terminal 
time. Now consider the matrix valued functions defined by

$$
A(x)=\sigma_{g}(T, x), \quad K(t, x)=\frac{\Phi(t, x)}{(T-t)^{2}}, \quad B(t, x)=K(t, x)+\frac{1}{2} H(x) .
$$

With these notations we have that

$$
\sigma_{P}(t, x)=(T-t) A(x)+(T-t)^{2} B(t, x)
$$

and therefore

$$
I(t, x) \equiv \operatorname{det} \sigma_{P}(t, x)=(T-t)^{d} \operatorname{det}(A(x)+(T-t) B(t, x)) .
$$

Well-known results from linear algebra show that for any two matrices $M_{1}$ and $M_{2}$ the determinant of the sum $M_{1}+M_{2}$ is given by

$$
\operatorname{det}\left(M_{1}+M_{2}\right)=\sum_{k=0}^{d} \sum_{1 \leq i_{1}<\cdots<i_{k} \leq d} \operatorname{det}\left(C_{i_{1}, \ldots, i_{k}}\right)
$$

where the matrix $C_{i_{1}, \ldots, i_{k}}$ is obtained from $M_{1}$ by replacing its rows with numbers $i_{1}, \ldots, i_{k}$ with the corresponding rows of $M_{2}$. Applying this formula to equation (42) we obtain

$$
\begin{aligned}
I(t, x) & =\sum_{k=0}^{d}(T-t)^{k+d} \sum_{1 \leq i_{1}<\cdots<i_{k} \leq d} \operatorname{det}\left(C_{i_{1}, \ldots, i_{k}}(t, x)\right) \\
& =(T-t)^{d} \operatorname{det} A(x)+\sum_{k=1}^{d}(T-t)^{k+d} \sum_{1 \leq i_{1}<\cdots<i_{k} \leq d} \operatorname{det}\left(C_{i_{1}, \ldots, i_{k}}(t, x)\right)
\end{aligned}
$$

where $C_{i_{1}, \ldots, i_{k}}(t, x)$ is obtained from $A(x)$ by replacing its rows with numbers $i_{1}, \ldots, i_{k}$ with the corresponding rows of the matrix $B(t, x)$. Expanding the second term on the right hand side and using the fact that

$$
\operatorname{det} C_{i}(t, x)=\frac{1}{2} \operatorname{det} B_{i}(x)
$$

for all $1 \leq i \leq d$ where the function $B_{i}$ is defined as in equation (14), we obtain that the determinant of the price gradient satisfies

$$
I(t, x)=(T-t)^{d}\left(\operatorname{det} A(x)+\frac{1}{2}(T-t) \sum_{i=1}^{d} \operatorname{det} B_{i}(x)\right)+o(T-t)^{1+d} .
$$

Finally, combining this expansion with an argument similar to that used in the proofs of Theorem 1 and 2 shows that $I(t, x) \neq 0$ for almost every $(t, x) \in(0, T) \times \mathcal{X}$ as soon as equation (15) is satisfied and the desired result now follows from Proposition 3. QED.

\section{C.3 Infinite horizon economies}

In order to establish Theorem 3 we will rely on the following lemma. 
Lemma C.1: Under the assumptions of Theorem 3 we have

$$
\begin{aligned}
& \lim _{\rho \rightarrow \infty} \rho \frac{\partial P}{\partial x}(x, \rho, \lambda)=\frac{\partial g}{\partial x}(x), \\
& \lim _{\rho \rightarrow \infty}\left(\rho^{2} \frac{\partial P}{\partial x}(x, \rho, \lambda)-\rho \frac{\partial g}{\partial x}(x)\right)=\frac{\partial}{\partial x} \frac{\mathcal{L}(\mathbf{m} g)(x, \lambda)}{\mathbf{m}(x, \lambda)}
\end{aligned}
$$

for all $x \in \mathcal{X}$.

Proof. By definition, the derivative of the function $Q_{i}=\mathbf{m} P_{i}$ with respect to the state variable $x_{j}$ is given by

$$
\begin{aligned}
\frac{\partial Q_{i}}{\partial x_{j}}(x, \rho, \lambda) & =\lim _{\varepsilon \rightarrow 0}\left(Q_{i}\left(x+\varepsilon \mathbf{e}_{j}, \rho, \lambda\right)-Q_{i}(x, \rho, \lambda)\right) / \varepsilon \\
& =\lim _{\varepsilon \rightarrow 0} \int_{0}^{\infty} e^{-\rho \tau}(1 / \varepsilon)\left(\pi_{i}\left(\tau, x+\varepsilon \mathbf{e}_{j}, \lambda\right)-\pi_{i}(\tau, x, \lambda)\right) d \tau .
\end{aligned}
$$

Fix an arbitrary $\bar{\varepsilon}>0$ and denote by $F_{1}$ the integrand on the right hand side of the above expression. Using the bound of Assumption E in conjunction with the continuity of the function $\Pi$ we obtain that for each fixed $x \in \mathcal{X}$ there exists a finite constant $C_{x, \lambda}$ such that

$$
\left|F_{1}(\varepsilon, \tau, x, \rho, \lambda)\right| \leq e^{(K-\rho) \tau} C_{x, \lambda}
$$

for all $\varepsilon \leq \bar{\varepsilon}$. Since $\rho>K$ by assumption, it now follows from the dominated convergence theorem that

$$
\frac{\partial Q_{i}}{\partial x_{j}}(x, \rho, \lambda)=\int_{0}^{\infty} e^{-\rho \tau} \frac{\partial \pi_{i}}{\partial x_{j}}(\tau, x, \lambda) d \tau
$$

and therefore

$$
\begin{aligned}
\frac{\partial P_{i}}{\partial x_{j}}(x, \rho, \lambda) & =\frac{1}{\mathbf{m}(x, \lambda)} \frac{\partial Q_{i}}{\partial x_{j}}(x, \rho, \lambda)-P(x, \rho, \lambda) \frac{\partial \log \mathbf{m}}{\partial x}(x, \lambda) \\
& =\int_{0}^{\infty} e^{-\rho \tau} \frac{\partial p_{i}}{\partial x_{j}}(\tau, x, \lambda) d \tau
\end{aligned}
$$

where we have set $p_{i} \equiv \pi_{i} / \mathbf{m}$ and the last equality follows from the definition of the price function and the chain rule. As a result, the first limit that we have to compute is given by

$$
A_{i j}(x, \lambda) \equiv \lim _{\rho \rightarrow \infty} \rho \frac{\partial P_{i}}{\partial x_{j}}(x, \rho, \lambda)=\lim _{\rho \rightarrow \infty} \int_{0}^{\infty} e^{-\theta} \frac{\partial p_{i}}{\partial x_{j}}(\theta / \rho, x, \lambda) d \theta
$$

where the second equality follows from the change of variable $\theta=\rho \tau$. Letting $F_{2}$ denote the integrand on the right hand side and relying once again on the bound of Assumption E we deduce that for each fixed $x \in \mathcal{X}$ there exists a finite constant $D_{x, \lambda}$ such that

$$
\left|F_{2}(\theta, x, \rho, \lambda)\right| \leq e^{(K / \rho-1) \theta} D_{x, \lambda} .
$$

Since $\rho>K$ by assumption, we may now apply the dominated convergence theorem in the definition of $A_{i j}$ to obtain

$$
A_{i j}(x, \lambda)=\lim _{\varepsilon \rightarrow 0} \frac{\partial p_{i}}{\partial x_{j}}(\varepsilon, x, \lambda)=\frac{\partial p_{i}}{\partial x_{j}}(0, x, \lambda)=\frac{\partial g_{i}}{\partial x_{j}}(x)
$$


where the second equality follows the definition of $p_{i}$ and the fact that the function $\pi$ belongs to $C^{1}\left(\mathbb{R}_{+} \times \mathcal{X}\right)$.

Using the above result in conjunction with the change of variable $\theta=\rho \tau$ we obtain that the second limit we have to compute is given by

$$
B_{i j}(x, \lambda)=\lim _{\rho \rightarrow \infty} \int_{0}^{\infty} e^{-\theta} \rho\left(\frac{\partial p_{i}}{\partial x_{j}}(\theta / \rho, x, \lambda)-\frac{\partial p_{i}}{\partial x_{j}}(0, x, \lambda)\right) d \theta
$$

Fix a strictly positive constant $\varepsilon$, let $\rho_{0}>K$ be arbitrary and denote by $F_{3}$ the integrand on the right hand side of the above expression. Using the bound of Assumption $\mathrm{E}$ we obtain that for each fixed $x \in \mathcal{X}$ there exists a finite constant $E_{x, \lambda}$ such that

$$
1_{\{\theta>\varepsilon \rho\}}\left|F_{3}(\theta, x, \rho, \lambda)\right| \leq 1_{\{\theta>\varepsilon \rho\}} \theta e^{\left(K / \rho_{0}-1\right) \theta} E_{x, \lambda}
$$

for all $\rho \geq \rho_{0}$. On the other hand, the definition of the derivative imply that for each fixed $x \in \mathcal{X}$ there exists a finite constant $\delta_{x, \lambda}$ such that

$$
1_{\{\theta \leq \varepsilon \rho\}}\left|F_{3}(\theta, x, \rho, \lambda)\right| \leq 1_{\{\theta \leq \varepsilon \rho\}} \theta e^{-\theta} \delta_{x, \lambda}\left|\frac{\partial^{2} p_{i}}{\partial t \partial x_{j}}(0, x, \lambda)\right| .
$$

Combining the two previous bounds we obtain that for each fixed $x \in \mathcal{X}$ there are finite constants $E_{x, \lambda}$ and $\Delta_{x, \lambda}$ such that

$$
\left|F_{3}(\theta, x, \rho, \lambda)\right| \leq \theta e^{-\theta}\left(\Delta_{x, \lambda}+\theta e^{\left(K / \rho_{0}\right) \theta} E_{x, \lambda}\right)
$$

for all $\rho \geq \rho_{0}>K$ and we may now apply the dominated convergence theorem in the definition of $B_{i j}$ to obtain

$$
B_{i j}(x, \lambda)=\frac{\partial^{2} p_{i}}{\partial t \partial x_{j}}(0, x, \lambda) .
$$

Since the function $\pi$ belongs to $C^{2}\left(\mathbb{R}_{+} \times \mathcal{X}\right)$ by assumption we know that it solves the partial differential equation

$$
\frac{\partial \pi}{\partial t}(\tau, x, \lambda)=\mathcal{L} \pi(\tau, x, \lambda)
$$

with terminal condition $\mathbf{m} g$. Using this property in conjunction with arguments similar to those we used in the finite horizon case we get

$$
\lim _{\tau \rightarrow 0} \frac{\partial p_{i}}{\partial t}(\tau, x, \lambda)=\frac{\mathcal{L}(\mathbf{m} g)(x, \lambda)}{\mathbf{m}(x, \lambda)}
$$

and the desired result follows from the fact that $\pi \in C^{2}\left(\mathbb{R}_{+} \times \mathcal{X}\right)$.

QED.

Proof of Theorem 3. By direct application of the result of Lemma C.1 we have that the volatility of the candidate prices satisfies

$$
\sigma_{P}(x, \rho, \lambda)=(1 / \rho) \sigma_{g}(x)+o(1 / \rho)
$$

and it follows that

$$
I(x, \rho, \lambda) \equiv \operatorname{det} \sigma_{P}(x, \rho, \lambda)=(1 / \rho)^{d} \operatorname{det} \sigma_{g}(x)+o(1 / \rho)^{d} .
$$

Now assume that equation (18) holds so that the function $I$ is not identically zero. Since 
$\partial P / \partial x$ is real analytic in $\rho$ by Assumption $\mathrm{E}$ we know that $I$ is also real analytic in $\rho$. Combining these properties with Lemma A.2 shows that there exists a countable set $\mathcal{R} \subseteq(K, \infty)$ outside of which $I(x, \rho, \lambda) \neq 0$ for almost every $x \in \mathcal{X}$ and the desired result now follows from Proposition 3.

QED.

Proof of Proposition 5. By direct application of the result of Lemma C.1 we have that the volatility of the candidate prices satisfies

$$
\sigma_{P}(x, \rho, \lambda)=(1 / \rho) \sigma_{g}(x)+(1 / \rho)^{2} F(x, \lambda)+o(1 / \rho)^{2} .
$$

Using this expression in conjunction with an argument similar to that we used in the proof of Proposition 4 then shows that we have

$$
\begin{aligned}
I(x, \rho, \lambda) & \equiv \operatorname{det} \sigma_{P}(x, \rho, \lambda) \\
& =(1 / \rho)^{d}\left(\operatorname{det} \sigma_{g}(x)+\frac{1}{2}(1 / \rho) \sum_{i=1}^{d} \operatorname{det} C_{i}(x, \lambda)\right)+o(1 / \rho)^{1+d} .
\end{aligned}
$$

where the functions $C_{i}$ are defined as in equation (19). Finally, combining this expansion with an argument similar to that used in the proofs of Theorem 3 shows that there exists a countable set $\mathcal{R} \subseteq(K, \infty)$ outside of which $I(x, \rho, \lambda) \neq 0$ for almost every $x \in \mathcal{X}$ and the result now follows from Proposition 3.

QED.

\section{Proofs for Section 4: Applications}

\section{D.1 Vector autoregressive processes}

In order to simplify the exposition we will assume throughout this section that $\bar{G}=$ $v_{a} \equiv 0$ for every $a$ and provide a detailed proof of Theorem 4 only under condition (b). The case where some of the risky securities pay terminal dividends is entirely analogous and even simpler since it only requires a first order expansion of the volatility of the candidate prices.

Lemma D.1: The transition density $p(t, x, \tau, y)$ of the state variables is real analytic in $(t, \tau) \in[0, T]^{2} \backslash\{t=\tau\}$ and there exists a complex neighborhood $\mathcal{P} \supseteq[0, T]$ with the following properties

(a) For every constant $\phi>0$ there exist a constant $H>0$ and a locally bounded function $L$ such that

$$
|p(z, x, z+\theta, y)| \leq L(x) e^{-H\|y-x\|^{2}}
$$

for all $(z, \theta, x, y) \in \mathcal{P} \times \mathbb{C} \times \mathcal{X}^{2}$ such that $z+\theta \in \mathcal{P}$ and $\Re(\theta)>\phi$.

(b) There exist a constant $C>0$ and a locally bounded function $J$ such that

$$
|p(z, x, z+\theta, y)| \leq|\theta|^{-d / 2} J(x) e^{-\frac{C}{\theta}\|y-x\|^{2}}
$$

for all $(z, \theta, x, y) \in \mathcal{P} \times \mathbb{R}_{+} \times \mathcal{X}^{2}$ such that $z+\theta \in \mathcal{P}$.

Proof. By the analytic implicit function theorem (see Lunardi (1995, Theorem 8.3.9)) we have that the unique solution to

$$
d \Phi(t)=-A(t) \Phi(t)
$$


with initial condition $\mathbb{I}_{d}$ is analytic in a neighborhood of $[0, T]$. Furthermore, the inverse of this unique solution solves

$$
d\left(\Phi^{-1}(t)\right)=\Phi^{-1}(t) A(t)
$$

with initial condition $\mathbb{I}_{d}$ and hence is analytic as well.

Using the above notation in conjunction with well-known results on linear SDEs (see Karatzas and Shreve (1998, Chapter 5.6.)) we obtain that the unique solution to equation (21) is a Gaussian process with mean

$$
\mu(t, x, \tau) \equiv E\left[X_{\tau} \mid X_{t}=x\right]=\Phi(\tau) \Phi^{-1}(t) x+\int_{t}^{\tau} \Phi(\tau) \Phi^{-1}(s) b(s) d s,
$$

and variance-covariance matrix

$$
\begin{aligned}
\Omega(t, \tau) & \equiv E\left[X_{\tau} X_{\tau}^{\top} \mid X_{t}\right]-E\left[X_{\tau} \mid X_{t}\right] E\left[X_{\tau} \mid X_{t}\right]^{\top} \\
& =\int_{t}^{\tau}\left(\Phi(\tau) \Phi^{-1}(s) \sigma_{X}(s)\right)\left(\Phi(\tau) \Phi^{-1}(s) \sigma_{X}(s)\right)^{\top} d s .
\end{aligned}
$$

In particular, the transition density of the state variables is given by

$$
p(t, x, \tau, y)=\phi(y, \mu(t, x, \tau), \Omega(t, \tau))
$$

where

$$
\phi(y, m, B) \equiv(2 \pi)^{-d / 2}|\operatorname{det}(B)|^{-1 / 2} e^{-(y-m)^{\top} B^{-1}(y-m)}
$$

denotes the $d$-dimensional Gaussian probability distribution function. Since the functions $b, \sigma_{X}, \Phi$ and $\Phi^{-1}$ are analytic we have that the functions $\mu$ and $\Omega$ are also analytic and it now follows from equation (45) that the transition density is real analytic in $(t, \tau) \in[0, T]^{2}$.

Since the horizon is finite and the matrix $\sigma_{X}(t)$ is by assumption nondegenerate for all $t \in[0, T]$ we know that there are strictly positive constants $\varepsilon$ and $\delta$ such that

$$
\delta \mathbb{I}_{d} \geq \Sigma_{X}(t) \equiv \sigma_{X}(t) \sigma_{X}(t)^{\top} \geq \varepsilon \mathbb{I}_{d}
$$

for all $t \in[0, T]$ where for two symmetric matrices $A \geq B$ means that $A-B$ is nonnegative definite. Now consider the function defined by

$$
\hat{\Omega}(t, \tau) \equiv \frac{\Omega(t, \tau)}{\tau-t} .
$$

Since the function $\Omega$ is analytic in a neighborhood of $[0, T]^{2}$ we know that the function $\hat{\Omega}$ is jointly real analytic for $t \neq \tau$. On the other hand, since

$$
\lim _{t \rightarrow \tau} \hat{\Omega}(t, \tau)=\Sigma_{X}(\tau)
$$

we have that the singularity at $\tau=t$ is removable and it follows that the function $\hat{\Omega}$ is real analytic in $(t, \tau) \in[0, T]^{2}$, see Shabat (1992, Theorem 3 p.92 and Hartog's Theorem p.28). Furthermore, the function $\hat{\Omega}$ satisfies

$$
\delta \mathbb{I}_{d} \geq \hat{\Omega}(t, \tau) \geq \varepsilon \mathbb{I}_{d}
$$


for all $(t, \tau) \in[0, T]^{2}$ and there exists a neighborhood $\mathcal{P} \supseteq[0, T]$ such that

$$
\|\hat{\Omega}(t, \tau)-\hat{\Omega}(\Re t, \Re \tau)\|<\frac{1}{2} \varepsilon
$$

for all $(t, \tau) \in \mathcal{P}^{2}$ where the notation $\|M\|$ denotes the Euclidean norm of the matrix $M$. Using these properties we readily obtain that

$$
\begin{aligned}
\|\hat{\Omega}(t, \tau)\| & \geq\|\hat{\Omega}(\Re t, \Re \tau)\|-\|\hat{\Omega}(t, \tau)-\hat{\Omega}(\Re t, \Re \tau)\| \\
& \geq \frac{h^{\top}}{\|h\|} \hat{\Omega}(\Re t, \Re \tau) \frac{h}{\|h\|}-\frac{1}{2} \varepsilon \geq \frac{1}{2} \varepsilon
\end{aligned}
$$

for any $h \in \mathbb{R}^{d}$ and it follows that

$$
\left\|\hat{\Omega}^{-1}(t, \tau)\right\| \leq \varepsilon_{1} \equiv \frac{2}{\varepsilon}
$$

for all $(t, \tau) \in \mathcal{P}^{2}$. Using similar arguments and setting $\xi(t, \tau) \equiv \mu(t, x, \tau)-\Phi(\tau) \Phi^{-1}(t) x$ it can be shown that the vector valued function

$$
\eta(t, x, \tau) \equiv \frac{x-\mu(t, x, \tau)}{\tau-t}=\frac{\left(\mathbb{I}_{d}-\Phi(\tau) \Phi^{-1}(t)\right)}{\tau-t} x-\frac{\xi(t, \tau)}{\tau-t}
$$

is real analytic in $(t, \tau) \in[0, T]^{2}$ and, since real analytic functions are locally bounded, it follows that there exists a constant $C_{0}>0$ such that

$$
\|\eta(t, x, \tau)\| \leq C_{0}(1+\|x\|)
$$

for all $(t, \tau, x) \in \mathcal{O}^{2} \times \mathcal{X}$.

After these lengthy preparations we now turn to the proof of assertions (a) and (b). Using the expression of the transition density in conjunction with equation (46) and the fact that

$$
\begin{aligned}
\frac{1}{|\operatorname{det}(\Omega(\tau, t))|^{1 / 2}} & =\left|\operatorname{det}\left(\Omega^{-1}(\tau, t)\right)\right|^{1 / 2} \leq\left\|\Omega^{-1}(t, \tau)\right\|^{d / 2} \\
& =\left\|(\tau-t)^{-1} \hat{\Omega}^{-1}(t, \tau)\right\|^{d / 2} \leq \varepsilon_{1}^{d / 2}|\tau-t|^{-d / 2}
\end{aligned}
$$

we deduce that

$$
|p(t, x, \tau, y)| \leq C_{p}|\tau-t|^{-d / 2} e^{-A_{1}+2 A_{2}+A_{3}}
$$

for all $(t, \tau, x, y) \in \mathcal{P}^{2} \times \mathcal{X}^{2}$ and some constant $C_{p}>0$ where

$$
\begin{aligned}
& A_{1}=(y-x)^{\top} \Re\left(\Omega^{-1}(t, \tau)\right)(y-x), \\
& A_{2}=\left|(y-x)^{\top} \Re\left(\hat{\Omega}^{-1}(t, \tau) \eta(t, x, \tau)\right)\right|, \\
& A_{3}=\left|\Re\left((\tau-t) \eta(t, x, \tau)^{\top} \hat{\Omega}^{-1}(t, \tau) \eta(t, x, \tau)\right)\right| .
\end{aligned}
$$


Using equations (46) and (47) we obtain that

$$
\begin{aligned}
A_{2} & \leq\|y-x\|\left\|\hat{\Omega}^{-1}(t, \tau) \eta(t, x, \tau)\right\| \\
& \leq\|y-x\|\left\|\hat{\Omega}^{-1}(t, \tau)\right\|\|\eta(t, x, \tau)\| \leq C_{2}(1+\|x\|)\|y-x\| \\
A_{3} & \leq|\tau-t|\left|\eta(t, x, \tau)^{\top} \hat{\Omega}^{-1}(t, \tau) \eta(t, x, \tau)\right| \\
& \leq|\tau-t|\left\|\hat{\Omega}^{-1}(t, \tau)\right\|\|\eta(t, x, \tau)\|^{2} \leq C_{3}(1+\|x\|)^{2}
\end{aligned}
$$

for all $(t, \tau, x, y) \in \mathcal{P}^{2} \times \mathcal{X}^{2}$ and some nonnegative constants $C_{i}$ where the last inequality follows from the boundedness of $\mathcal{P}$. Now let $\phi>0$ be a fixed constant. Since $\hat{\Omega}^{-1}(t, \tau) \geq$ $\varepsilon_{2}$ and $\Im\left(\hat{\Omega}^{-1}(t, \tau)\right)=0$ for some constant $\varepsilon_{2}>0$ and all real $(t, \tau)$ we can assume by shrinking the neighborhood if necessary that

$$
\Re\left(\hat{\Omega}^{-1}(\tau, t)\right) \geq \frac{1}{2} \varepsilon_{2} \mathbb{I}_{d}
$$

and

$$
\left\|\Im(\tau-t) \Im\left(\hat{\Omega}^{-1}(t, \tau)\right)\right\| \leq \frac{\phi}{4} \varepsilon_{2}
$$

for all $(t, \tau) \in \mathcal{P}^{2}$. Using these estimates we deduce that there exists a strictly positive constant $C_{1} \equiv C_{1}(\phi)$ such that

$$
\begin{aligned}
A_{1} & =\frac{(y-x)^{\top}\left(\Re(\tau-t) \Re\left(\hat{\Omega}^{-1}(t, \tau)\right)+\Im(\tau-t) \Im\left(\hat{\Omega}^{-1}(t, \tau)\right)\right)(y-x)}{|\tau-t|^{2}} \\
& \geq \frac{4(y-x)^{\top} \Re(\tau-t) \Re\left(\hat{\Omega}^{-1}(t, \tau)\right)(y-x)-\phi \varepsilon_{2}\|y-x\|^{2}}{4|\tau-t|^{2}} \\
& \geq \frac{2 \phi \varepsilon_{2}\|y-x\|^{2}-\phi \varepsilon_{2}\|y-x\|^{2}}{4|\tau-t|^{2}} \geq C_{1}\|y-x\|^{2}
\end{aligned}
$$

for all $(t, \tau, x, y) \in \mathcal{P}^{2} \times \mathcal{X}^{2}$ such that $\Re(\tau-t) \geq \phi$ where the last inequality follows from the boundedness of $\mathcal{P}$. Combining equations (49), (50) and (52) with the elementary inequality

$$
a^{2}+b^{2} \geq a b, \quad \forall(a, b) \in \mathbb{R}_{+} \times \mathbb{R}_{+},
$$

we finally obtain that

$$
A_{3}+2 A_{2}-A_{1} \leq-\frac{C_{1}}{2}\|y-x\|^{2}+\left(C_{3}+8 \frac{C_{2}^{2}}{C_{1}}\right)(1+\|x\|)^{2}
$$

for all $(t, \tau, x, y) \in \mathcal{P}^{2} \times \mathcal{X}^{2}$ such that $\Re(\tau-t) \geq \phi$ and assertion (a) now follows from equation (48) and the boundedness of the set $\mathcal{P}$.

In order to complete the proof fix an arbitrary pair $(t, \tau) \in \mathcal{P}^{2}$ such that $\theta \equiv \tau-t \in$ $\mathbb{R}_{+}$. In this case, equation (51) implies that

$$
A_{1}=\frac{1}{\theta}(y-x)^{\top} \Re\left(\hat{\Omega}^{-1}(t, \tau)\right)(y-x) \geq \frac{\varepsilon_{2}}{2 \theta}\|y-x\|^{2}
$$

for all $(x, y) \in \mathcal{X}^{2}$ and assertion (b) now follows from equations (48), (49), (50) and the elementary inequality (53). We omit the details.

QED. 
LEMMA D.2: The candidate price function $P:[0, T] \times \mathcal{X} \rightarrow \mathbb{R}_{+}^{d}$ is well-defined and real analytic in $t \in(0, T)$.

Proof. Fix an arbitrary $t_{0} \in(0, T)$. By Proposition 2 we know that the function $m>0$ is real analytic with respect to $t \in[0, T]$ and so it suffices to prove that for each $i$ the function

$$
\begin{aligned}
Q_{i}(z, x) & \equiv m(z, x) P_{i}(z, x) \\
& =\int_{0}^{T-z} \int_{\mathcal{X}} p(z, x, z+\theta, y)\left(m g_{i}\right)(z+\theta, y) d y d \theta
\end{aligned}
$$

is well-defined and analytic in a complex neighborhood $\mathcal{P}_{0} \supset t_{0}$. To this end we decompose the above integral as $Q_{i 1}+Q_{i 2}$ where

$$
\begin{aligned}
Q_{i 1}(z, x) & \equiv \int_{0}^{T-t_{0}} \int_{\mathcal{X}} p(z, x, z+\theta, y)\left(m g_{i}\right)(z+\theta, y) d y d \theta \\
Q_{i 2}(z, x) & \equiv \int_{T-t_{0}}^{T-z} \int_{\mathcal{X}} p(z, x, z+\theta, y)\left(m g_{i}\right)(z+\theta, y) d y d \theta
\end{aligned}
$$

Let $\mathcal{P}_{0}=\mathcal{P} \cap \mathcal{B}$ where $\mathcal{P} \supseteq[0, T]$ is the complex neighborhood whose existence is asserted in Lemma D.1 and $\mathcal{B}$ is a disk of radius $\varepsilon<t_{0} \vee\left(T-t_{0}\right)$ centered at the point $t_{0}$. Using equation (43) in conjunction with the definition of the constant $\varepsilon$ and the assumption that $f \in \mathcal{K}$ we obtain that the integrand in the definition of $Q_{i 2}$ satisfies

$$
\left|p(z, x, z+\theta, y)\left(m g_{i}\right)(z+\theta, y)\right| \leq K L(x) e^{K\|y\|^{\alpha}-H\|y-x\|^{2}}
$$

for some locally bounded function $L$ and some strictly positive constants $K, H$ and $\alpha<2$. Since the right hand side of the above inequality is integrable with respect to $y$ we have that $Q_{i 2}(z, x)$ is well defined for all $z \in \mathcal{P}_{0}$. On the other hand, using equation (54) in conjunction with Fubini's theorem and the fact that both $p$ and $m g_{i}$ are real analytic in time we obtain that

$$
\int_{\Gamma} Q_{i 2}(\gamma, x) d \gamma=0
$$

for any closed contour $\Gamma \subseteq \mathcal{P}_{0}$ and analyticity of $Q_{i 2}$ with respect to $z \in \mathcal{P}_{0}$ now follows from Morera's theorem (see Shabat (1992, Theorem 2 p.85)).

By shrinking the set $\mathcal{B}$ if necessary we can assume that $z+\theta \in \mathcal{P}$ for all $(z, \theta) \in$ $\mathcal{P}_{0} \times \mathbb{R}_{+}$such that $\theta \leq T-t_{0}$. Using this property in conjunction with equation (44) and the assumption that $f \in \mathcal{K}$ we obtain that

$$
\left|p(z, x, z+\theta, y)\left(m g_{i}\right)(z+\theta, y)\right| \leq K J(x)|\theta|^{-d / 2} e^{K\|y\|^{\alpha}-\frac{C}{|\theta|}\|y-x\|^{2}}
$$

for some locally bounded function $L$ and some strictly positive constants $C, K$ and $\alpha<2$. Since the right hand side of the above inequality is integrable with respect to $y$ we have that $Q_{i 1}(z, x)$ is well defined for all $z \in \mathcal{P}_{0}$. On the other hand, using equation (55) in conjunction with Fubini's theorem and the fact that both $p$ and $m g_{i}$ are real analytic in time we obtain that

$$
\int_{\Gamma} Q_{i 1}(\gamma, x) d \gamma=\int_{0}^{T-t_{0}} \int_{\mathcal{X}} \int_{\Gamma} p(\gamma, x, \gamma+\theta, y)\left(m g_{i}\right)(\gamma+\theta, y) d \gamma d y d \theta=0
$$

for any closed contour $\Gamma \subseteq \mathcal{P}_{0}$ and analyticity of $Q_{i 1}$ with respect to $z \in \mathcal{P}_{0}$ now follows 
Lemma D.3: The candidate price function $P:[0, T] \times \mathcal{X} \rightarrow \mathbb{R}_{+}^{d}$ is real analytic in $x \in \mathcal{X}$ and belongs to $C^{3}((0, T] \times \mathcal{X})$.

Proof. By Proposition 2 we know that $m>0$ is real analytic in $x \in \mathcal{X}$ and so it suffices to prove the result for the function $Q \equiv m P$.

In order to deal with the function $Q_{i}$ fix two open sets $\mathcal{X}_{1} \subset \mathcal{X}_{2} \subseteq \mathcal{X}$, assume that $x \in \mathcal{X}_{1}$ and let

$$
h_{i}(z, x) \equiv N(x)\left(m g_{i}\right)(z, x)
$$

where $N: \mathcal{X} \rightarrow[0,1]$ is an arbitrary smooth function which is equal to 1 on the set $\mathcal{X}_{1}$ and to zero outside of the set $\mathcal{X}_{2}$. Using this notation we may decompose the function $Q_{i}$ as

$$
Q_{i}(z, x)=H_{i}(z, x)+F_{i}(z, x)
$$

where

$$
\begin{aligned}
& H_{i}(t, x) \equiv \int_{0}^{T-t} \int_{\mathcal{X}_{2}} p(t, x, t+\theta, y) h_{i}(t+\theta, y) d y d \theta \\
& F_{i}(t, x) \equiv \int_{0}^{T-t} \int_{\mathcal{X}_{1}^{c}} p(t, x, t+\theta, y)\left(m g_{i}-h_{i}\right)(t+\theta, y) d y d \theta
\end{aligned}
$$

and $\mathcal{X}_{1}^{c} \equiv \mathcal{X} \backslash \mathcal{X}_{1}$. Using the assumption that $f \in \mathcal{K}$ in conjunction with arguments similar to those in the proof of Lemma D.1 it can be shown that

$$
\left|\frac{\partial^{k} p(t, x, \tau, y)}{\partial^{k_{1}} x_{1} \cdots \partial^{k_{d}} x_{d}}\left(m g_{i}-h_{i}\right)(\tau, y)\right| \leq C_{k 2}\left(C_{k 3}+\|x\|+\|y\|\right)^{k} e^{-\frac{C_{k 2}}{2 T}\|y-x\|^{2}}
$$

for all $(t, \tau, y) \in[0, T]^{2} \times \mathcal{X}_{1}^{c}$ and some strictly positive constants $C_{k 2}, C_{k 3}$ which depend on the choice of $x \in \mathcal{X}_{1}$. Since the right hand side of the above estimate is integrable with respect to $(t, y)$ it follows from standard results on the differentiation of integrals that $F_{i}$ is smooth and satisfies

$$
\frac{\partial^{k} F_{i}(t, x)}{\partial^{k_{1}} x_{1} \cdots \partial^{k_{d}} x_{d}}=\int_{0}^{T-t} \int_{\mathcal{X}} \frac{\partial^{k} p(t, x, t+\theta, y)}{\partial^{k_{1}} x_{1} \cdots \partial^{k_{d}} x_{d}}\left(m g_{i}-h_{i}\right)(t+\theta, y) d y d \theta
$$

for all $(t, x) \in[0, T] \times \mathcal{X}_{1}$. Furthermore, since the bound in equation (56) is uniform with respect to the time argument we may apply the dominated convergence theorem in the above expression to obtain

$$
\lim _{t \rightarrow T} \frac{\partial^{k} F_{i}(t, x)}{\partial^{k_{1}} x_{1} \cdots \partial^{k_{d}} x_{d}}=0
$$

Summarizing the above results we have thus far proved that the function $F_{i}$ belongs to $C^{0, \infty}\left((0, T] \times X_{1}\right)$. Combining this result with that of Lemma D.2 and the fact that the process

$$
F_{i}\left(t, X_{t}\right)+\int_{0}^{t}\left(m g_{i}-h_{i}\right)\left(\tau, X_{\tau}\right) d \tau=E_{t}\left[\int_{0}^{T}\left(m g_{i}-h_{i}\right)\left(\tau, X_{\tau}\right) d \tau\right]
$$


is a martingale we obtain that the function $F_{i}$ is a classical solution to the partial differential equation

$$
-\frac{\partial F_{i}}{\partial t}(t, x)=\left(m g_{i}-h_{i}\right)(t, x)+\mathcal{L} F_{i}(t, x)
$$

Now consider the function $H_{i}$. Since the integrand $h_{i}$ is smooth and compactly supported, it follows from standard Feynman-Kac arguments Oksendal (2007, Theorem 8.2.1) that the function $H_{i}$ is smooth and solves the partial differential equation ${ }^{28}$

$$
-\frac{\partial H_{i}}{\partial t}(t, x)=h_{i}(t, x)+\mathcal{L} H_{i}(t, x) .
$$

Furthermore, using the fact that the derivatives of $h_{i}$ are all uniformly bounded in conjunction with a straightforward change of variables we obtain

$$
\begin{aligned}
& \frac{\partial^{k} H_{i}(t, x)}{\partial^{k_{1}} x_{1} \cdots \partial^{k_{d}} x_{d}}= \\
& \int_{0}^{T-t} \int_{\mathcal{X}} e^{-\frac{\|z\|^{2}}{2}} \frac{\partial^{k} h_{i}}{\partial^{k_{1}} x_{1} \cdots \partial^{k_{d}} x_{d}}\left(\tau, \Omega(t, \tau)^{1 / 2} z+\mu(t, x, \tau)\right) d z d \theta
\end{aligned}
$$

and it now follows from the dominated convergence theorem that

$$
\lim _{t \rightarrow T} \frac{\partial^{k} H_{i}(t, x)}{\partial^{k_{1}} x_{1} \cdots \partial^{k_{d}} x_{d}}=0
$$

Adding equations (58) and (59), we get that the function $Q_{i}$ is a solution to the partial differential equation

$$
-\frac{\partial Q_{i}}{\partial t}(t, x)=\left(m g_{i}\right)(t, x)+\mathcal{L} Q_{i}(t, x)
$$

and since all the coefficients in this partial differential equation equation are real analytic it now follows from Theorem A.1 that the function $Q_{i}$ is real analytic in $x \in \mathcal{X}$ and that its radius of complex analyticity is uniformly bounded from below when $t$ varies in compact subsets of $(0, T)$.

On the other hand, adding equations (57) and (60) we obtain that all the space derivatives of the function $Q_{i}$ converge to zero as $t \rightarrow T$ and the proof will be complete once we show that the derivatives

$$
\frac{\partial Q_{i}}{\partial t}, \quad \frac{\partial^{2} Q_{i}}{\partial t^{2}}, \quad \frac{\partial^{2} Q_{i}}{\partial t \partial x_{j}}, \quad \frac{\partial^{3} Q_{i}}{\partial t^{3}}, \quad \frac{\partial^{3} Q_{i}}{\partial t^{2} \partial x_{j}} \quad \text { and } \frac{\partial^{3} Q_{i}}{\partial t \partial x_{j} \partial x_{k}}
$$

are all continuous at the terminal time. The continuity of $\partial Q_{i} / \partial t$ follows from equation (61), Assumption A and the fact that $Q_{i} \in C^{0, \infty}((0, T] \times \mathcal{X})$ by application of the first part of the proof. On the other hand, differentiating both sides of equation (61) with respect to the space variables gives

$$
-\frac{\partial^{k+1} Q_{i}}{\partial t \cdot \partial x_{1}^{k_{1}} \cdots \partial x_{d}^{k_{d}}}(t, x)=\frac{\partial^{k}\left(m g_{i}+\mathcal{L} Q_{i}\right)}{\partial x_{1}^{k_{1}} \cdots \partial x_{d}^{k_{d}}}(t, x)
$$

for all $k \in \mathbb{N}$ and it follows that $Q_{i} \in C^{1, \infty}((0, T] \times \mathcal{X})$ since we know from Assumption

\footnotetext{
${ }^{28}$ It can also be easily checked that the function $H_{i}$ is a weak solution to this PDE and is therefore a strong solution by hypoellipticity. See Shikamura (1992, Theorem 2.1 p.99)
} 
A, Proposition 2 and the first part of the proof that the right hand side is continuous at the terminal time. Similarly, differentiating both sides of equation (61) with respect to time we obtain

$$
-\frac{\partial^{2} Q_{i}}{\partial t^{2}}(t, x)=\frac{\partial\left(m g_{i}\right)}{\partial t}(t, x)+\mathcal{L} \frac{\partial Q_{i}}{\partial t}(t, x)+\widehat{\mathcal{L}} Q_{i}(t, x)
$$

where

$$
\widehat{\mathcal{L}} \equiv\left(b^{\prime}(t)-A^{\prime}(t) x\right) \frac{\partial}{\partial x}+\sum_{i, j=1}^{n} \frac{1}{2}\left(\Sigma_{X}^{\prime}(t)\right)_{i j} \frac{\partial^{2}}{\partial x_{i} \partial x_{j}}
$$

and the continuity of the second order time derivative follows from Assumption A, Proposition 2 and the fact that $Q_{i} \in C^{1, \infty}((0, T] \times \mathcal{X})$. The continuity of the third order time derivative can be established similarly by differentiating equation (62) first with respect to $x$ and then with respect to time. We omit the details.

QED.

Proof of Theorem 4. Combining Lemma D.2, Lemma D.3, Theorem A.1 and Theorem A.2 we obtain that the candidate price function is real analytic in $(t, x) \in[0, T] \times \mathcal{X}$ and belongs to $C^{3}((0, T] \times \mathcal{X})$. The desired result now follows from Theorem 2 and Proposition 4.

QED.

In order to establish Propositions 6,7 and 8 we will rely on the following more general result:

Proposition 10: Consider a finite horizon economy, let $\xi: \mathcal{X} \rightarrow \mathbb{R}_{+}$be a given function and assume that

(a) Agent a has subjective rate of time preference $\rho_{a} \geq 0$ and his utility functions are given by

$$
u_{a}(c)=v_{a}(c) \equiv \frac{c^{1-\gamma_{a}}-1}{1-\gamma_{a}}
$$

for some strictly positive relative risk aversion coefficient $\gamma_{a}$.

(b) The dividends of stock $i$ are given by equations (24) and (25) for some constant $\delta_{i} \in \mathbb{R}$ and some functions $\left(\nu_{i}, \kappa_{i}\right)$ such that

$$
e^{-J_{i} \xi(x)} J_{i} \leq \nu_{i}(x), \kappa_{i}(x) \leq e^{K_{i} \xi(x)} K_{i}
$$

for some strictly positive constants $\left(J_{i}, K_{i}\right)$.

Then there exists a complex neighborhood $\mathcal{O} \supset[0, T]$ with the property that the function $f$ of equation (23) can be analytically continued to $\mathcal{O}$ for all $x \in \mathcal{X}$ and satisfies the bound

$$
|f(\tau, x)| \leq e^{\xi(x) L} L
$$

for all $(\tau, x) \in \mathcal{O} \times \mathcal{X}$ and some strictly positive constant $L$.

Proof. Assume without loss of generality that $\bar{G} \equiv 0$, let $b_{a} \equiv 1 / \gamma_{a}$ and consider the function

$$
\phi(x, z, u) \equiv \sum_{a=1}^{A} e^{-\rho_{a} b_{a} z} u^{-b_{a}} c_{a 0}-\bar{g}(z, x) .
$$


With this notation we have that the state price density solves $\phi(x, z, m(z, x))=0$ and we want to apply the analytic implicit function theorem to obtain analyticity of $m(z, x)$ with respect to $z \in \mathcal{O}$ for some complex neighborhood $\mathcal{O} \supset[0, T]$ which is independent of the choice of $x \in \mathcal{X}$.

For real values of $t \in[0, T]$ we have that

$$
\max _{1 \leq a \leq A}\left(e^{-\rho_{a} b_{a} z} u^{-b_{a}} c_{a 0}\right) \geq \sum_{a=1}^{A} e^{-\rho_{a} b_{a} z}\left(c_{a 0} / A\right) u^{-b_{a}} \geq e^{-\rho_{i} b_{i} z} u^{-b_{i}}\left(c_{i 0} / A\right)
$$

for any index $1 \leq i \leq A$ and it follows that there exists a strictly positive constant $C_{1}$ such that

$$
\left(1 / C_{1}\right) \sum_{a=1}^{A} \bar{g}(t, x)^{-\gamma_{a}} \leq m(t, x) \leq C_{1} \sum_{a=1}^{A} \bar{g}(t, x)^{-\gamma_{a}} .
$$

By Theorem 1.1 of Chang, He, and Pradhu (2003) we know that the radius of time analyticity of the function $m$ depends only on two quantities: a lower bound on $|\partial \phi / \partial u|$ at the point $(x, t, m(t, x))$ and an upper bound on $|\phi(x, z, u)|$ for $(x, z, u)$ in a complex neighborhood of the point $(x, t, m(t, x)) \cdot{ }^{29}$

By compactness, when $\bar{g}(t, x)$ varies in fixed bounded interval the claim follows from the analytic implicit function theorem. Now we have to consider two regimes: that where $\bar{g}(t, x)$ is large and that where it is small. Assume first that $\bar{g}(t, x)>K_{4}$ for some sufficiently large constant $K_{4}$, let $\gamma_{\min } \equiv \min _{a} \gamma_{a}$ and consider the function $\ell(z, x) \equiv$ $m(z, x) \bar{g}(z, x)^{\gamma_{\min }}$. By equation (64) we have that there strictly positive constants $C_{2}$ and $C_{3}$ such that

$$
C_{2}+C_{2} \sum_{\gamma_{a}>\gamma_{\min }}^{A} \bar{g}(t, x)^{\gamma_{\min }-\gamma_{a}} \leq m(t, x) \leq C_{3}+C_{3} \sum_{\gamma_{a}>\gamma_{\min }}^{A} \bar{g}(t, x)^{\gamma_{\min }-\gamma_{a}}
$$

and it follows that $\ell(z, x)$ varies in a bounded interval as long as the aggregate dividend is bounded away from zero. On the other hand, a direct calculation shows that $\ell(z, x)$ solves $\psi(x, z, \ell(z, x))=0$ where

$$
\psi(z, x, u) \equiv \sum_{a=1}^{A} e^{-\rho_{a} b_{a} z} \bar{g}(z, x)^{\frac{\gamma_{\min }}{\gamma_{a}}-1} u^{-b_{a}} c_{a 0}-1
$$

In particular, we have that

$$
\frac{\partial \psi(z, x, u)}{\partial u} \geq-b_{i} e^{-\rho_{i} b_{i} z} u^{-b_{i}} c_{i 0}
$$

where $i=\arg \min _{a} \gamma_{a}$ denotes the agent with the smallest risk aversion and it follows that the derivative is uniformly bounded away from zero as $u=\ell(z, x)$ is bounded away from zero. Since

$$
|\bar{g}(z, x)| \geq|\Re \bar{g}(z, x)| \geq K_{5}|\bar{g}(\Re z, x)| \geq K_{4} K_{5}
$$

for some strictly positive constant $K_{5}$ we can make $\bar{g}(z, x)$ arbitrary large by increasing the constant $K_{4}$. Sending $\bar{g}(z, x)$ to infinity and using the identity $\psi(z, x, \ell(z, x))=0$

\footnotetext{
${ }^{29}$ Even though the conditions of Chang et al. (2003) only require a bound on $|\phi(x, t, u)|$ for real values of $t$ a close inspection of their proof shows that one in fact needs a bound on $|\phi(x, z, u)|$ for complex values of $z$.
} 
in conjunction with the fact that $\ell(z, x)$ stays uniformly bounded we obtain

$$
\lim _{\bar{g}(z, x) \rightarrow \infty} \sum_{\gamma_{a}>\gamma_{\min }}^{A} e^{-\rho_{a} b_{a} z} \bar{g}(z, x)^{\frac{\gamma_{\min }}{\gamma_{a}}-1} u^{-b_{a}} c_{a 0}=0
$$

and therefore

$$
\lim _{\bar{g}(z, x) \rightarrow \infty} \ell(z, x)=e^{-\rho_{i} z} c_{i 0}^{\gamma_{\min }}
$$

where the convergence is uniform in $x \in \mathcal{X}$ such that $\bar{g}(t, x) \geq K_{4}$ for all $t \in[0, T]$ and some sufficiently large $K_{4}$. Hence, $|\psi(z, x, u)|$ can be made arbitrarily small when $|u-\ell(t, x)|+|z-t|<\varepsilon$ uniformly in $x$ for some sufficiently small $\varepsilon$ and it follows that the radius of time analyticity of $\ell(z, x)$, which is equal to that of $m(z, x)$, is uniformly bounded from below when the aggregate dividend is large.

In the regime where the aggregate dividend is small the desired result follows from a similar argument using the function $s(z, y) \equiv m(z, x) \bar{g}(z, x)^{\gamma_{\max }}$ with $\gamma_{\max } \equiv \max _{a} \gamma_{a}$. We omit the details.

To complete the proof, let $\mathcal{O} \supseteq[0, T]$ denote the domain of complex analyticity of $m(z, x)$. Using the definition of the state price density in conjunction with the triangle inequality we obtain that

$$
|\bar{g}(z, x)| \leq K_{6} \sum_{a=1}^{A}|m(z, x)|^{-b_{a}}
$$

for some strictly positive constant $K_{6}$ and it follows that there exists an index $a \equiv a(x)$ and a strictly positive constant $K_{7}$ such that

$$
|m(z, x)|^{-b_{a(x)}} \geq \frac{|\bar{g}(z, x)|}{A K_{6}} \geq \frac{|\Re \bar{g}(z, x)|}{A K_{6}} \geq K_{7} \sum_{i=1}^{n} \kappa_{i}(x) .
$$

Combining this estimate with the lower bound of equation (63) we obtain that there exists a strictly positive constant $K_{8}$ such that $|m(z, x)| \leq K_{8} e^{\xi(x) K_{8}}$ for all $(z, x) \in$ $\mathcal{O} \times \mathcal{X}$ and the desired result now follows from the upper bound of equation (63). QED.

Proof of Proposition 6. The desired result follows from Proposition 10 after setting $\xi(x)=\|x\|^{\alpha}$ for some $\alpha \in(0,2)$.

QED.

Proof of Theorem 5. To facilitate the presentation of the proof we distinguish two cases: that where the matrix $A$ is diagonalizable and has eigenvalues with nonnegative real parts; and that where all its eigenvalues have strictly positive real parts.

Case 1. The desired result will follow from those of Theorem 3 and Proposition 5 once we show that the function

$$
\pi_{i}(\tau, x) \equiv E\left[\mathbf{m}\left(X_{\tau}\right) g_{i}\left(X_{\tau}\right) \mid X_{0}=x\right]=\int_{\mathbb{R}^{n}} p(0, x, \tau, y)\left(\mathbf{m} g_{i}\right)(y) d y
$$

is twice continuously differentiable with respect to the state variables and satisfies the conditions of Assumption E. The smoothness of $\pi_{i}$ follows from arguments similar to those we used in the proof of Lemma D.3 so all we have to prove is that $\pi_{i}$ and its first two derivatives satisfy the bounds in equation (17). 
By assumption we have that $A=B D B^{-1}$ for some diagonal matrix $D$ whose elements have nonnegative real part and it follows that the norm of

$$
\|\Phi(\tau)\|=\left\|B e^{-D \tau} B^{-1}\right\| \leq K_{0}
$$

for all $\tau>0$ and some strictly positive constant $K_{0}$. Using this property it is not hard to see that we have

$$
\begin{aligned}
\left\|\Omega(\tau)^{1 / 2}\right\| & \leq K_{1} \sqrt{\tau}, \\
\|\mu(0, x, \tau)\| & \leq K_{1}(\tau+\|x\|),
\end{aligned}
$$

for all $\tau>0$ and some strictly positive constant $K_{1}$ where

$$
\Omega(\tau)=\int_{0}^{\tau}\left(\Phi(\tau) \Phi(s)^{-1} \sigma_{X}\right)\left(\Phi(\tau) \Phi(s)^{-1} \sigma_{X}\right)^{\top} d s
$$

is the variance-covariance matrix of the state variables over an interval of length $\tau$. On the other hand, the assumption of the statement imply that we have

$$
\left|\left(\mathbf{m} g_{i}\right)(y)\right| \leq C e^{C\|y\|}
$$

for some strictly positive constant $C$ and using this inequality in conjunction with equation (65), equation (66), the definition of the transition density and a straightforward change of variables we obtain

$$
\begin{aligned}
\left|\pi_{i}(\tau, x)\right| & =\frac{1}{(2 \pi)^{n / 2}} \int_{\mathbb{R}^{n}} e^{-\|z\|^{2} / 2}\left(\mathbf{m} g_{i}\right)\left(\Omega(\tau)^{1 / 2} z+\mu(0, x, \tau)\right) d z \\
& \leq \frac{1}{(2 \pi)^{n / 2}} \int_{\mathbb{R}^{n}} e^{-\|z\|^{2} / 2} C e^{C\left(\Omega(\tau)^{1 / 2} z+\mu(0, x, \tau)\right)} d z \\
& \leq \frac{1}{(2 \pi)^{n / 2}} \int_{\mathbb{R}^{n}} e^{-\|z\|^{2} / 2} C e^{K_{2}(\|x\|+\sqrt{\tau}\|z\|+\tau)} d z \\
& \leq C e^{K_{2}(\|x\|+\tau)} \prod_{k=1}^{n} \frac{1}{(2 \pi)^{n / 2}} \int_{\mathbb{R}^{n}} e^{-\|z\|^{2} / 2}\left(e^{\sqrt{\tau} K_{2}\|z\|}+e^{-\sqrt{\tau} K_{2}\|z\|}\right) d z \\
& =2^{n} C e^{K_{2}\|x\|+K_{2}\left(1+K_{2} / 2\right) \tau} \leq K_{3} e^{K_{2}\|x\|+K_{3} \tau},
\end{aligned}
$$

for all $x \in \mathcal{X}$ and some strictly positive constants $K_{2}, K_{3}$. This shows that $\pi_{i}$ satisfies the bound of Assumption $\mathrm{E}$ and it now only remains to show that its first two space derivatives satisfy a similar bound.

Fix an arbitrary $x \in \mathcal{X}$, set $f_{i} \equiv \mathbf{m} g_{i}$ and let $\tilde{f}_{i}$ denote a smooth, compactly supported function which agrees with the function $f_{i}$ in a neighborhood of $x$. Using this notation we decompose the function $\pi_{i}$ as $\pi_{i}=\tilde{\pi}_{i}+\hat{\pi}_{i}$ where

$$
\begin{aligned}
& \tilde{\pi}_{i}(\tau, x) \equiv \int_{\mathbb{R}^{n}} p(0, x, \tau, y) \tilde{f}_{i}(y) d y, \\
& \hat{\pi}_{i}(\tau, x) \equiv\left(\pi_{i}-\tilde{\pi}_{i}\right)(\tau, x)=\int_{\mathbb{R}^{n}} p(0, x, \tau, y)\left(f_{i}-\tilde{f}_{i}\right)(y) d .
\end{aligned}
$$

Since $\tilde{f}_{i}$ is smooth and compactly supported we know that $\tilde{f}_{i}$ as well as all of its derivatives are uniformly bounded and combining this property with the same change 
of variable as above we obtain that

$$
\begin{aligned}
\left|\frac{\partial^{k} \tilde{\pi}_{i}(\tau, x)}{\partial^{k_{1}} x_{1} \cdots \partial^{k_{n}} x_{n}}\right| & \leq C_{k} \int_{\mathbb{R}^{n}} e^{-\frac{\|z\|^{2}}{2}}\left|\frac{\partial^{k} \tilde{f}_{i}}{\partial^{k_{1}} x_{1} \cdots \partial^{k_{n}} x_{n}}\left(\Omega(\tau)^{1 / 2} z+\mu(0, x, \tau)\right)\right| d z \\
& \leq C_{k}^{\prime}\|\Phi(\tau)\|^{k} \leq C_{k}^{\prime \prime}
\end{aligned}
$$

for all $\tau>0$ and some strictly positive constants $C_{k}, C_{k}^{\prime}$ where the last inequality follows from the uniform boundedness of $\|\Phi\|$.

Finally, consider the functioon $\hat{\pi}_{i}$. Fix an $\varepsilon>0$. Since the matrix $\Omega(\tau)$ is nondegenerate for all strictly positive $\tau>0$ and is monotone increasing in $\tau$ we have that there exists a strictly positive constant $K_{4}$ such that $\Omega(\tau)>K_{4} \mathbb{I}_{n}$ for all $\tau>\varepsilon$ and it follows that $|\operatorname{det} \Omega(\tau)| \geq K_{4}^{n}$. Combining this inequality with an induction argument it is now easily seen that

$$
\left|\frac{\partial^{k} p(0, x, \tau, y)}{\partial^{k_{1}} x_{1} \cdots \partial^{k_{n}} x_{n}}\right| \leq \tau^{k} K_{5}\left(\|x\|^{k}+\|y\|^{k}\right) e^{-(y-\mu(0, x, \tau))^{\top} \Omega(\tau)^{-1}(y-\mu(0, x, \tau))}
$$

for some strictly positive constant $K_{5}$ and it now follows from the assumption of the statement that

$$
\begin{aligned}
& \left|\frac{\partial^{k} \hat{\pi}_{i}(\tau, x)}{\partial^{k_{1}} x_{1} \cdots \partial^{k_{n}} x_{n}}\right| \\
& \quad \leq \tau^{k} K(x) \int_{\mathbb{R}^{n}}\left(1+\|y\|^{k}\right) e^{-(y-\mu(0, x, \tau))^{\top} \Omega(\tau)^{-1}(y-\mu(0, x, \tau))} e^{C\|y\|} d y .
\end{aligned}
$$

for some nonnegative function $K$. Using the same change of variable as above in conjunction with equation (65), equation (66) and the fact that power functions grow slower than exponentials we obtain that

$$
\begin{aligned}
\left|\frac{\partial^{k} \hat{\pi}_{i}(\tau, x)}{\partial^{k_{1}} x_{1} \cdots \partial^{k_{n}} x_{n}}\right| & \leq \tau^{k} K(x) \int_{\mathbb{R}^{n}} e^{-(y-\mu(0, x, \tau))^{\top} \Omega(\tau)^{-1}(y-\mu(0, x, \tau))} e^{K_{6}\|y\|} d y \\
& \leq e^{K_{8} \tau} L(x) \int_{\mathbb{R}^{n}} e^{-\|z\|^{2} / 2} e^{\sqrt{\tau} K_{7}\|z\|} d z \\
& \leq e^{K_{9} \tau} L(x)
\end{aligned}
$$

for some strictly positive constants $\left(K_{i}\right)_{i=6}^{9}$ and some nonnegative function $L$ where the last inequality follows from an argument similar to that which led to equation (67). This shows that the first two space derivatives of the function $\hat{\pi}_{i}$ satisfy the bound of Assumption E and completes the proof for Case 1.

CASE 2. When all the eigenvalues of the matrix $A$ have strictly positive real parts the proof proceeds in exactly the same way except that the norms of the functions $\Omega(\tau)$ and $\mu(0, x, \tau)$ are now uniformly bounded with respect to the time argument instead of having linear growth. We omit the details.

QED.

\section{D.2 Autonomous diffusion processes}

In order to simplify the exposition we will assume throughout this appendix that $\bar{G}=$ $v_{a} \equiv 0$ for every $a$ and provide a detailed proof under condition (b). The case where some of the risky securities pay terminal dividends is entirely analogous and even simpler. 
Fix an arbitrary point $c$ in the interior of the state space and denote by

$$
s_{i}(x) \equiv \int_{c_{i}}^{x} \exp \left(-2 \int_{c_{i}}^{\xi} \frac{\mu_{i}(\theta)}{\sigma_{i}^{2}(\theta)} d \theta\right) d \xi
$$

the scale function associated with the $i$-th coordinate of the vector of state variables. Then the process

$$
Y_{i t} \equiv s_{i}\left(X_{i t}\right)=Y_{i 0}+\int_{0}^{t} \frac{1}{a_{i}\left(Y_{i \tau}\right)} d Z_{i \tau}
$$

with

$$
\frac{1}{a_{i}(y)} \equiv s_{i}^{\prime}\left(s_{i}^{-1}(y)\right) \sigma_{i}\left(s^{-1}(y)\right)
$$

is a nonnegative local martingale and a straightforward change of variables implies the following:

Lemma D.4: (a) Assumption $G$ holds if and only if the transition density $\tilde{q}_{i}$ of the process $Y_{i}$ satisfies

$$
\tilde{q}_{i}(t, x, y) \leq I_{i}(x) J_{i}(y) t^{-\alpha_{i}} e^{-\left(\tilde{C}_{i} / t\right)\left(A_{i}(y)-A_{i}(x)\right)^{2}}
$$

for all $(t, x, y) \in[0, T] \times \mathbb{R}_{+}^{2}$, some strictly positive constants $\left(\alpha_{i}, \tilde{C}_{i}\right)$ and some locally bounded functions $\left(I_{i}, J_{i}\right)$ where we have set

$$
A_{i}(y) \equiv \int_{0}^{y} a_{i}(z) d z .
$$

(b) A real analytic function $f:[0, T] \times \mathcal{X} \rightarrow \mathbb{R}$ belongs to the class $\mathcal{M}$ if and only if the function

$$
\tilde{f}(\tau, y) \equiv f\left(\tau, s_{1}^{-1}\left(y_{1}\right), \ldots, s_{d}^{-1}\left(y_{d}\right)\right)
$$

satisfies the inequality

$$
\int_{\mathbb{R}_{+}^{d}} \sup _{\tau \in \mathcal{O}}|\tilde{f}(\tau, y)| \prod_{i=1}^{d} J_{i}\left(y_{i}\right) e^{-\varepsilon A_{i}\left(y_{i}\right)} d y<\infty
$$

for any $\varepsilon>0$ and some complex neighborhood of the time interval $(0, T)$.

Relying on the above lemma we will from now assume that the state variable $X_{i} \equiv Y_{i}$ evolves according to equation (68) instead of equation (30) and that its transition density $q_{i} \equiv \tilde{q}_{i}$ satisfies equation (69). Based on these assumptions we redefine $\mathcal{M}$ as the class of those real analytic functions $\tilde{f}$ which satisfy the conditions of the second part of Lemma D.4. Note that since the coefficient in equation (30) are all real analytic the functions $a_{i}$ which determine the volatility of the new state variables are also real analytic.

The following lemma shows that the bound of equation (69) also holds for complex values of the time argument and will be crucial in order to prove that the candidate prices are real analytic in time.

Lemma D.5: The transition density $q_{i}$ is real analytic in $(t, x, y) \in \mathbb{R}_{+} \times \mathcal{X}_{i}^{2}$ and the following assertions hold: 
(a) For every real $\tau>0$ there exists a complex neighborhood $\mathcal{P} \supset \tau$ such that

$$
\left|q_{i}(z, x, y)\right| \leq B_{i} I_{i}(x) J_{i}(y) \tau^{-\alpha_{i}} e^{-\left(C_{i} / \tau\right)\left(A_{i}(y)-A_{i}(x)\right)^{2}}
$$

for all $(z, x, y) \in \mathcal{P} \times \mathcal{X}_{i}^{2}$ and some strictly positive constants $\left(B_{i}, C_{i}\right)$.

(b) For each $k \in \mathbb{N}$ there exist a strictly positive constant $C_{i k}>0$ such that

$$
\left|\frac{\partial^{k} q_{i}}{\partial t^{k}}(t, x, y)\right| \leq C_{i k} I_{i}(x) J_{i}(y) t^{-\left(\alpha_{i}+k\right)} e^{-\left(B_{i} / t\right)\left(A_{i}(y)-A_{i}(x)\right)^{2}}
$$

for all $(t, x, y) \in[0, T] \times \mathcal{X}_{i}^{2}$ where the strictly positive constant $B_{i}$ is the same as in assertion (a).

Proof. The function $q_{i}(t, x, y)$ solves both the forward and the backward Kolmogorov equation and thus is real analytic in $(y, x) \in \mathcal{X}_{i}^{2} \backslash\{x=y\}$ by application of Theorem A.1. On the other hand, since $X_{i}$ is a local martingale we know that $q_{i}(t, x, y)$ is the heat kernel associated with the differential operator

$$
\mathcal{L}_{i} \equiv \frac{1}{2 a_{i}^{2}(y)} \frac{\partial^{2}}{\partial x_{i}^{2}}
$$

and the remaining claims thus follow from Davies (1997, Theorem 3 and 4) after suitable modifications.

QED.

Lemma D.6: The candidate price function $P:[0, T] \times \mathcal{X} \rightarrow \mathbb{R}_{+}^{d}$ is well-defined and real analytic in $t \in(0, T)$.

Proof. By Proposition 2 we know that the function $m>0$ is real analytic in $t \in[0, T]$ and so it suffices to prove the result for the function $Q \equiv m P$. Since the state variables are mutually independent we have that

$$
Q_{i}(z, x) \equiv \int_{0}^{T-z} \int_{\mathcal{X}} p(\theta, x, y)\left(m g_{i}\right)(z+\theta, y) d y d \theta
$$

where

$$
p(t, x, y) \equiv \prod_{i=1}^{d} q_{i}\left(t, x_{i}, y_{i}\right)
$$

is the transition density of the vector of state variables. Now fix an arbitrary $t_{0} \in(0, T)$. We need to show that the above integral is well-defined and analytic with respect to $z$ in a complex neighborhood $\mathcal{P}_{0} \supset t_{0}$. To this end, we decompose the above integral as $Q_{i 1}+Q_{i 2}$ where

$$
\begin{aligned}
Q_{i 1}(z, x) & \equiv \int_{0}^{T-t_{0}} \int_{\mathcal{X}} p(\theta, x, y)\left(m g_{i}\right)(z+\theta, y) d y d \theta \\
Q_{i 2}(z, x) & \equiv \int_{T-t_{0}}^{T-z} \int_{\mathcal{X}} p(\theta, x, y)\left(m g_{i}\right)(z+\theta, y) d y d \theta
\end{aligned}
$$

Let $\tau \equiv T-t_{0}$ and $\mathcal{P}_{0}=T-\mathcal{P} \supset t_{0}$ where $\mathcal{P}$ is the complex neighborhood whose existence is asserted in Lemma D.5. Using the upper bound in (70) together with the 
elementary inequality

$$
(a-b)^{2}-\frac{a^{2}}{2}+b^{2} \geq 0, \quad \forall(a, b) \in \mathbb{R} \times \mathbb{R},
$$

we deduce that the integrand in the definition of $Q_{i 2}$ satisfies

$$
\begin{aligned}
\left|p(\theta, x, y)\left(m g_{i}\right)(z+\theta, y)\right| & \leq \hat{f}(y) \prod_{i=1}^{d} I_{i}\left(x_{i}\right) J_{i}\left(y_{i}\right) \tau^{-\alpha_{i}} e^{-\frac{B_{i}}{\tau}\left(A_{i}\left(y_{i}\right)-A_{i}\left(x_{i}\right)\right)^{2}} \\
& \leq \hat{f}(y) K(x) \prod_{i=1}^{d} J_{i}\left(y_{i}\right) e^{-\frac{B_{i}}{2 \tau} A_{i}\left(y_{i}\right)^{2}}
\end{aligned}
$$

for some locally bounded function $K$ where

$$
\hat{f}(y) \equiv \sup _{z \in \mathcal{O}}|f(z, y)|
$$

and $\mathcal{O}$ is the complex neighborhood of $(0, T)$ whose existence is postulated in Definition 4. Since $f \in \mathcal{M}$ we have that the right hand side of equation (73) with respect to $y$ and it follows that $Q_{i 2}(z, x)$ is well-defined for all $z \in \mathcal{P}_{0}$. On the other hand, using equation (73) in conjunction with Fubini's theorem and the fact that both $p$ and $m g_{i}$ are real analytic we obtain that

$$
\int_{\Gamma} Q_{i 2}(\gamma, x) d \gamma=0
$$

for any closed contour $\Gamma \subseteq \mathcal{P}_{0}$ and analyticity of $Q_{i 2}$ with respect to $z \in \mathcal{P}_{0}$ now follows from Morera's theorem (see Shabat (1992, Theorem 2 p.85)).

In order to deal with the function $Q_{i 1}$ fix two small open sets $\mathcal{X}_{1} \subset \mathcal{X}_{2} \subseteq \mathcal{X}$, assume that $x \in \mathcal{X}_{1}$ and let

$$
h_{i}(z, x) \equiv N(x)\left(m g_{i}\right)(z, x)
$$

where $N: \mathcal{X} \rightarrow[0,1]$ is an arbitrary smooth function which is equal to 1 on the set $\mathcal{X}_{1}$ and to zero outside of the set $\mathcal{X}_{2}$. Using this notation we may decompose the function $Q_{i 1}$ as

$$
Q_{i 1}(z, x)=H_{i 1}(z, x)+F_{i 1}(z, x)
$$

where

$$
\begin{aligned}
& H_{i 1}(z, x) \equiv \int_{0}^{T-t_{0}} \int_{\mathcal{X}_{2}} p(\theta, x, y) h_{i}(z+\theta, y) d y d \theta, \\
& F_{i 1}(z, x) \equiv \int_{0}^{T-t_{0}} \int_{\mathcal{X}_{1}^{c}} p(\theta, x, y)\left(m g_{i}-h_{i}\right)(z+\theta, y) d y d \theta,
\end{aligned}
$$

and $\mathcal{X}_{1}^{c} \equiv \mathcal{X} \backslash \mathcal{X}_{1}$. Consider first the function $F_{i 1}$. Since we are integrating over $\mathcal{X}_{1}^{c}$ we know that $\|y-x\| \geq \varepsilon$ for some strictly positive constant $\varepsilon$ and it now follows from the continuity of the functions $A_{i}$ that

$$
W(x, y) \equiv \sum_{i=1}^{d} B_{i}\left(A_{i}\left(y_{i}\right)-A_{i}\left(x_{i}\right)\right)^{2} \geq \delta
$$


for all $y \in \mathcal{X}_{1}^{c}$ for some strictly positive constant $\delta \equiv \delta(x)$. Using this estimate in conjunction with the fact that power functions grow slower than exponentials we obtain that

$$
\theta^{-\sum_{i=1}^{n} \alpha_{i}} e^{-\frac{1}{\theta} W(x, y)} \leq C e^{\frac{\delta}{2 \theta}-\frac{1}{\theta} W(x, y)} \leq C e^{-\frac{1}{2 T} W(x, y)}
$$

for all $(\theta, x, y) \in[0, T] \times \mathcal{X}_{1} \times \mathcal{X}_{1}^{c}$ and some constant $C>0$. Combining this estimate with the elementary inequality (72) and the bound of Assumption G we deduce that

$$
\begin{aligned}
\left|p(\theta, x, y)\left(m g_{i}-h_{i}\right)(z+\theta, y)\right| & \leq 2 \hat{f}(y) \prod_{i=1}^{d} I_{i}\left(x_{i}\right) J_{i}\left(y_{i}\right) \theta^{-\alpha_{i}} e^{-\frac{1}{\theta} W(x, y)} \\
& \leq 2 C \hat{f}(y) \prod_{i=1}^{d} I_{i}\left(x_{i}\right) J_{i}\left(y_{i}\right) e^{-\frac{1}{2 T} W(x, y)} \\
& \leq C^{\prime} \hat{f}(y) \prod_{i=1}^{d} J_{i}\left(y_{i}\right) e^{-\frac{B_{i}}{4 T} A_{i}\left(y_{i}\right)^{2}}
\end{aligned}
$$

for all $(z, \theta, x, y) \in \mathcal{P}_{0} \times[0, T] \times \mathcal{X}_{1} \times \mathcal{X}_{1}^{c}$ and some strictly positive constant $C^{\prime} \equiv C^{\prime}(x)>$ 0 . Since $f \in \mathcal{M}$ by assumption we know that the right hand side of the above inequality is integrable with respect to $y$ and it follows that $F_{i 1}$ is well-defined on $\mathcal{P}_{0}$. On the other hand, using equation (75) in conjunction with Fubini's theorem and the fact that the functions $p, m g_{i}$ and $h_{i}$ are all real analytic in time we obtain that

$$
\int_{\Gamma} F_{i 1}(\gamma, x) d \gamma=0
$$

for any closed contour $\Gamma \subseteq \mathcal{P}_{0}$ and analyticity of the function $F_{i 1}$ with respect to $z \in \mathcal{P}_{0}$ now follows from Morera's theorem.

In order to complete the proof it remains to show that $H_{i 1}$ is well-defined and analytic with respect to $z \in \mathcal{P}_{0}$. Since we are integrating over a compact set we know that $\left|h_{i}\right| \leq \hat{f}$ is uniformly bounded. This implies that

$$
\left|p(\theta, x, y) h_{i}(z+\theta, y)\right| \leq C_{i}(x) p(\theta, x, y)
$$

for all $(z, \theta, x, y) \in \mathcal{P}_{0} \times[0, T] \times \mathcal{X}_{1} \times \mathcal{X}_{2}$ and locally bounded $C_{i}(x)>0$. In particular, we have

$$
\left|H_{i 1}(z, x)\right| \leq C_{i}(x)\left(T-t_{0}\right)
$$

for all $(z, x) \in \mathcal{P}_{0} \times \mathcal{X}_{1}$ and it follows that the function $H_{i 1}$ is well-defined on $\mathcal{P}_{0}$. On the other hand, using equation (76) in conjunction with Fubini's theorem the fact that both $p$ and $m g_{i}$ are real analytic we obtain that

$$
\int_{\Gamma} H_{i 1}(\gamma, x) d \gamma=0
$$

for any closed contour $\Gamma \subseteq \mathcal{P}_{0}$ and the desired analyticity follows once again from Morera's theorem.

QED.

Lemma D.7: Let $a<b$ and assume that $h \in C^{2}[a, b]$. Then

$$
\sup _{x \in[a, b]}\left|h^{\prime}(x)\right| \leq K\left(\sup _{x \in[a, b]}|h(x)|+\sup _{x \in[a, b]}\left|h^{\prime \prime}(x)\right|\right)
$$


for some strictly positive constant $K \equiv K(a, b)$.

Proof. Let $x<(b-a) / 2$ and fix $y \geq(b-a) / 2$ such that $x+y \in[a, b]$. By application of Taylor's formula we know that

$$
h(x+y)=h(x)+y f^{\prime}(x)+\frac{1}{2}|y|^{2} h^{\prime \prime}(\xi)
$$

for some constant $\xi \equiv \xi(x, y) \in[a, b]$. Using this expression in conjunction with the triangle inequality we deduce that

$$
\begin{aligned}
\left|h^{\prime}(x)\right| & \leq \frac{|y|}{2}\left|h^{\prime \prime}(\xi)\right|+\frac{1}{|y|}(|h(x)|+|h(x+y)|) \\
& \leq \frac{|y|}{2} \sup _{x \in[a, b]}\left|h^{\prime \prime}(x)\right|+\frac{2}{|y|} \sup _{x \in[a, b]}|h(x)| \\
& \leq \frac{|b-a|}{2} \sup _{x \in[a, b]}\left|h^{\prime \prime}(x)\right|+\frac{4}{|b-a|} \sup _{x \in[a, b]}|h(x)|
\end{aligned}
$$

for all $x<(b-a) / 2$. A similar argument with $y \leq(a-b) / 2$ shows that the same inequality holds for all $x>(b-a) / 2$ and the desired result now follows by taking the supremum with respect to $x$ on the left hand side.

QED.

Lemma D.8: Fix two open sets $\mathcal{X}_{1} \subset \mathcal{X}_{2} \subset \mathcal{X}$ such that

$$
\operatorname{dist}\left(\partial \mathcal{X}_{1}, \partial \mathcal{X}_{2}\right)>0
$$

and let $\mathcal{X}_{2}^{c} \equiv \mathcal{X} \backslash \mathcal{X}_{2}$. Then for every fixed $k \in \mathbb{N}$ there exists a strictly positive constant $C_{k}>0$ such that

$$
\sup _{x \in \mathcal{X}_{1}}\left|\frac{\partial^{k} p}{\partial x_{1}^{k_{1}} \cdots \partial x_{d}^{k_{d}}}(t, x, y)\right| \leq C_{k} \prod_{i=1}^{d} J_{i}\left(y_{i}\right) e^{-\frac{B_{i}}{4 T} A_{i}\left(y_{i}\right)^{2}}
$$

for all $(t, y) \in[0, T] \times \mathcal{X}_{2}^{c}$ and multi-indices $\left(k_{1}, \ldots, k_{d}\right)$ of length at most $k$.

Proof. Using equation (71) we obtain

$$
\frac{\partial^{k} p(t, x, y)}{\partial x_{1}^{k_{1}} \cdots \partial x_{d}^{k_{d}}}=\prod_{i=1}^{d} \frac{\partial^{k_{i}} q_{i}}{\partial x_{i}^{k_{i}}}\left(t, x_{i}, y_{i}\right)
$$

and so it suffices to prove the result for the $i$-th coordinate. Since the sets in which $x$ and $y$ vary are separated we know that $\|x-y\| \geq \varepsilon$ for some strictly positive $\varepsilon$ and it follows from the continuity of the function $A_{i}$ that

$$
B_{i}\left(A_{i}\left(y_{i}\right)-A_{i}\left(x_{i}\right)\right)^{2} \geq \delta
$$

for all $(x, y) \in \mathcal{X}_{1} \times \mathcal{X}_{2}^{c}$ and some strictly positive constant $\delta$. Using this estimate in conjunction with inequalities (69), (72) and (74) we obtain

$$
\begin{aligned}
\left|q_{i}\left(t, x_{i}, y_{i}\right)\right| & \leq I_{i}\left(x_{i}\right) J_{i}\left(y_{i}\right) t^{-\alpha_{i}} e^{-\frac{B_{i}}{t}\left(A_{i}\left(y_{i}\right)-A_{i}\left(x_{i}\right)\right)^{2}} \\
& \leq I_{i}\left(x_{i}\right) J_{i}\left(y_{i}\right) e^{-\frac{B_{i}}{4 t} A_{i}\left(y_{i}\right)^{2}+\frac{B_{i}}{2 t} A_{i}\left(x_{i}\right)^{2}} \\
& \leq K_{i 0} J_{i}\left(y_{i}\right) e^{-\frac{B_{i}}{4 T} A_{i}\left(y_{i}\right)^{2}}
\end{aligned}
$$


for all $(t, x, y) \in[0, T] \times \mathcal{X}_{1} \times \mathcal{X}_{2}^{c}$ where the last inequality follows from the local boundedness of the functions $I_{i}$ and $A_{i}$.

This shows that the statement holds for $k=0$. In order to prove that it also holds for $k>0$ recall that the transition density is a smooth solution to the backward Kolmogorov equation

$$
\frac{\partial^{2} q_{i}}{\partial x_{i}^{2}}\left(t, x_{i}, y_{i}\right)=2 a_{i}\left(x_{i}\right)^{2} \mathcal{L}_{i}\left(q_{i}\left(t, x_{i}, y_{i}\right)\right)=2 a_{i}\left(x_{i}\right)^{2} \frac{\partial q_{i}}{\partial t}\left(t, x_{i}, y_{i}\right)
$$

for all $x \neq y$ and $t>0$ and, hence, is real analytic in $x$ and $y$ for $x \neq y$ by application of Using this property Eidelman (1969, Theorem 6.2 p.221). Using (78) in conjunction with the second part of Lemma D.5 and an argument similar to that which led to equation (77) we obtain that

$$
\begin{aligned}
\left|\frac{\partial^{2} q_{i}}{\partial x_{i}^{2}}\left(t, x_{i}, y_{i}\right)\right| & \leq 2 a_{i}\left(x_{i}\right)^{2} C_{i 1} I_{i}\left(x_{i}\right) J_{i}\left(y_{i}\right) t^{-\left(1+\alpha_{i}\right)} e^{-\frac{B_{i}}{t} A_{i}\left(x_{i}, y_{i}\right)^{2}} \\
& \leq C_{i 2} J_{i}\left(y_{i}\right) e^{-\frac{B_{i}}{4 T} A_{i}\left(y_{i}\right)^{2}}
\end{aligned}
$$

for all $(t, x, y) \in[0, T] \times \mathcal{X}_{1} \times \mathcal{X}_{2}^{c}$

This shows that the statement holds for $k=2$ and, since we know from the first part that it holds for $k=0$, we may apply Lemma D.7 to conclude that it also holds for $k=1$. Differentiating both side of the partial differential equation equation (78) with respect to time we get

$$
\frac{\partial^{3} q_{i}}{\partial t \partial x_{i}^{2}}\left(t, x_{i}, y_{i}\right)=2 a_{i}\left(x_{i}\right)^{2} \frac{\partial^{2} q_{i}}{\partial t^{2}}\left(t, x_{i}, y_{i}\right)
$$

and the same argument as above shows that

$$
\left|\frac{\partial^{2} q_{i}}{\partial t \partial x_{i}}\left(t, x_{i}, y_{i}\right)\right|+\left|\frac{\partial^{3} q_{i}}{\partial t \partial x_{i}^{2}}\left(t, x_{i}, y_{i}\right)\right| \leq C J_{i}\left(y_{i}\right) e^{-\frac{B_{i}}{4 T} A_{i}\left(y_{i}\right)^{2}} .
$$

for all $(t, x, y) \in[0, T] \times \mathcal{X}_{1} \times \mathcal{X}_{2}^{c}$ and some strictly positive constant $C$. Now, differentiating equation (78) with respect to $x_{i}$ gives

$$
\frac{\partial^{3} q_{i}}{\partial x_{i}^{3}}\left(t, x_{i}, y_{i}\right)=4 a_{i}^{\prime}\left(x_{i}\right) a_{i}\left(x_{i}\right) \frac{\partial q_{i}}{\partial t}\left(t, x_{i}, y_{i}\right)+2 a_{i}\left(x_{i}\right)^{2} \frac{\partial^{2} q_{i}}{\partial t \partial x_{i}}\left(t, x_{i}, y_{i}\right)
$$

and combining this identity with equation (80) and the real analyticity of the function $a_{i}$ we conclude that the statement holds for $k=3$. The rest of the proof is done in a similar way by repeatedly differentiating equation (79) with respect to $t$ and $x_{i}$ and then applying Lemma D.7. We omit the details.

QED.

Lemma D.9: The candidate price function $P:[0, T] \times \mathcal{X} \rightarrow \mathbb{R}_{+}^{d}$ is real analytic in $x \in \mathcal{X}$ and belongs to $C^{3}((0, T] \times \mathcal{X})$.

Proof. By Proposition 2 we know that the function $m>0$ is real analytic in $x \in \mathcal{X}$ and so it suffices to prove the result for the function $Q \equiv m P$.

In order to deal with the function $Q_{i}$ fix again two small open sets $\mathcal{X}_{1} \subset \mathcal{X}_{2} \subseteq \mathcal{X}$, assume that $x \in \mathcal{X}_{1}$ and let

$$
h_{i}(z, x) \equiv N(x)\left(m g_{i}\right)(z, x)
$$


where $N: \mathcal{X} \rightarrow[0,1]$ is a smooth function which is equal to 1 on the set $\mathcal{X}_{1}$ and to zero outside of the set $\mathcal{X}_{2}$. Using this notation we may decompose the function $Q_{i 1}$ as

$$
Q_{i}(z, x)=H_{i}(z, x)+F_{i}(z, x)
$$

where

$$
\begin{aligned}
H_{i}(z, x) & \equiv \int_{0}^{T-t_{0}} \int_{\mathcal{X}_{2}} p(\theta, x, y) h_{i}(z+\theta, y) d y d \theta, \\
F_{i}(z, x) & \equiv \int_{0}^{T-t_{0}} \int_{\mathcal{X}_{1}^{c}} p(\theta, x, y)\left(m g_{i}-h_{i}\right)(z+\theta, y) d y d \theta,
\end{aligned}
$$

and $\mathcal{X}_{1}^{c} \equiv \mathcal{X} \backslash \mathcal{X}_{1}$. Using the result of Lemma D.8 we obtain that

$$
\left|\frac{\partial^{k} p(\theta, x, y)}{\partial^{k_{1}} x_{1} \cdots \partial^{k_{d}} x_{d}}\left(m g_{i}\right)(\tau, y)\right| \leq C_{k 2} \hat{f}(y) \prod_{i=1}^{d} J_{i}\left(y_{i}\right) e^{-\frac{B_{i}}{4 T} A_{i}\left(y_{i}\right)^{2}}
$$

for all $(\tau, \theta, y) \in[0, T]^{2} \times \mathcal{X}_{1}^{c}$ and some strictly positive constant $C_{k 2}$ which depends on the choice of $x$ but stays uniformly bounded when $x$ varies in compact subsets of $\mathcal{X}_{1}$. Since the right hand side of the above estimate is integrable with respect to $(y, \theta)$ it follows from standard results on the differentiation of integrals that the function $F_{i}$ is smooth with respect to $x$ and satisfies

$$
\frac{\partial^{k} F_{i}(t, x)}{\partial^{k_{1}} x_{1} \cdots \partial^{k_{d}} x_{d}}=\int_{0}^{T-t} \int_{\mathcal{X}_{1}^{c}} \frac{\partial^{k} p(\theta, x, y)}{\partial^{k_{1}} x_{1} \cdots \partial^{k_{d}} x_{d}}\left(m g_{i}-h_{i}\right)(t+\theta, y) d y d \theta
$$

for all $t \in[0, T]$ and $x$ varying in compact subsets of $\mathcal{X}_{1}$. Furthermore, it follows from the above bounds that there exists a constant $K>0$ such that

$$
\left|\frac{\partial^{k}}{\partial x_{i}^{k}} q_{i}\left(t, x_{i}, y_{i}\right)\right| \leq K e^{-1 /(K t)}
$$

for all $i$ and and $y \in \mathcal{X}_{1}^{c}$ when $x$ varies on compact subsets of $\mathcal{X}_{1}$. Thus, we may apply the dominated convergence theorem in equation (81) to obtain

$$
\lim _{t \rightarrow T} \frac{\partial^{k} F_{i}(t, x)}{\partial^{k_{1}} x_{1} \cdots \partial^{k_{d}} x_{d}}=0
$$

Summarizing the above results we have thus far proved that the function $F_{i}$ belongs to $C^{0, \infty}\left((0, T] \times X_{1}\right)$. Combining this result with that of Lemma D. 6 shows that $F_{i}$ is a classical solution to the partial differential equation

$$
-\frac{\partial F_{i}}{\partial t}(t, x)=\left(m g_{i}-h_{i}\right)(t, x)+\sum_{j=1}^{d} \frac{1}{2 a_{j}\left(x_{j}\right)^{2}} \frac{\partial^{2} F_{i}}{\partial x_{j}^{2}}(t, x) .
$$

Now consider the function $H_{i}$. Since the integrand $h_{i}$ is smooth and compactly supported it follows from standard Feynman-Kac arguments (see, e.g., Oksendal (2007, Theorem 
8.2.1)) that the function $H_{i}$ is a smooth solution to the partial differential equation ${ }^{30}$

$$
-\frac{\partial H_{i}}{\partial t}(t, x)=h_{i}(t, x)+\sum_{j=1}^{d} \frac{1}{2 a_{j}\left(x_{j}\right)^{2}} \frac{\partial^{2} H_{i}}{\partial x_{j}^{2}}(t, x) .
$$

Adding (83) and (84), we get that

$$
-\frac{\partial Q_{i}}{\partial t}(t, x)=\left(m g_{i}\right)(t, x)+\sum_{j=1}^{d} \frac{1}{2 a_{j}\left(x_{j}\right)^{2}} \frac{\partial^{2} Q_{i}}{\partial x_{j}^{2}}(t, x)
$$

and since all the coefficients in this partial differential equation are real analytic it now follows from Theorem A.1 that $Q_{i}$ is real analytic in $(t, x) \in(0, T) \times \mathcal{X}$ and that its radius of complex analyticity is uniformly bounded from below when $t$ varies in compact subsets of $(0, T)$.

In order to prove that the function $Q_{i}$ belongs to $C^{3}((0, T] \times \mathcal{X})$ and thus complete the proof the first step is to show that

$$
\lim _{t \rightarrow T} \frac{\partial^{k} Q_{i}(t, x)}{\partial^{k_{1}} x_{1} \cdots \partial^{k_{d}} x_{d}}=0
$$

for all multi-indices of length at most 3 . As shown by equation (82) this property holds for the function $F_{i}$ and since $Q_{i}=F_{i}+H_{i}$ we only need to establish it for the function $H_{i}$. First of all, using the fact that the transition density converges to a point mass as $t \rightarrow T$ we obtain

$$
\lim _{t \rightarrow T} H_{i}(t, x)=\lim _{t \rightarrow T} \int_{0}^{T-t} \int_{\mathcal{X}_{2}} p(\theta, x, y) h_{i}(t+\theta, y) d y d \theta=0 .
$$

On the other hand, since the semigroup of the state variables commutes with the action of the generator $\mathcal{L}=\sum_{j=1}^{d} \mathcal{L}_{j}$ and the individual generators $\mathcal{L}_{j}$ are pairwise commuting we obtain

$$
\begin{aligned}
\mathcal{L}_{j} H_{i}(t, x)=\mathcal{L}_{j} \int_{0}^{T-t} \int_{\mathcal{X}_{2}} p(\theta, x, y) & h_{i}(t+\theta, y) d y d \theta s \\
= & \int_{0}^{T-t} \int_{\mathcal{X}_{2}} p(\theta, x, y) \mathcal{L}_{j} h_{i}(t+\theta, y) d y d \theta
\end{aligned}
$$

and the fact that the transition density converges to a point mass as time to maturity decreases to zero now implies that

$$
\lim _{t \rightarrow T} \mathcal{L}_{j} H_{i}(t, x)=\lim _{t \rightarrow T} \frac{1}{2 a_{j}\left(x_{j}\right)^{2}} \frac{\partial^{2} H_{i}(t, x)}{\partial^{2} x_{j}}=0 .
$$

for every $j$. Using this property in conjunction with the smoothness of $a_{j}$, equation (86) and the result of Lemma D.7 we obtain that

$$
\lim _{t \rightarrow T} \frac{\partial^{2} H_{i}(t, x)}{\partial x_{j}}=\lim _{t \rightarrow T} \frac{\partial H_{i}(t, x)}{\partial x_{j}}=0
$$

Similarly, applying $\mathcal{L}_{k}$ on both sides of equation (87) and using the fact that the

\footnotetext{
${ }^{30}$ It can also be easily checked that $H_{i}$ is a weak solution to this partial differential equation and is therefore a strong solution by hypoellipticity. See Shikamura (1992, Theorem 2.1 p.99).
} 
transition density converges to a point mass we obtain that

$$
\lim _{t \rightarrow T} \mathcal{L}_{k} \mathcal{L}_{j} H_{i}(t, x)=\lim _{t \rightarrow T} \frac{1}{4 a_{k}\left(x_{k}\right)^{2} a_{j}\left(x_{j}\right)^{2}} \frac{\partial^{4} H_{i}(t, x)}{\partial^{2} x_{j} \partial^{2} x_{k}}=0
$$

and combining this property with equation (89) and the result of Lemma D.7 we conclude that

$$
\lim _{t \rightarrow T} \frac{\partial^{4} H_{i}(t, x)}{\partial x_{j}^{2} \partial x_{k}^{2}}=\lim _{t \rightarrow T} \frac{\partial^{3} H_{i}(t, x)}{\partial x_{j}^{2} \partial x_{k}}=0 .
$$

Now, using the result of Lemma D.7 together with the second parts of equations (89) and (90) we obtain that

$$
\lim _{t \rightarrow T} \frac{\partial^{2} H_{i}(t, x)}{\partial x_{j} \partial x_{k}}=0
$$

and the validity of equation (85) will follow once we prove that

$$
\lim _{t \rightarrow T} \frac{\partial^{3} H_{i}(t, x)}{\partial x_{j} \partial x_{k} \partial x_{l}}=0
$$

for all $j \neq k \neq l$. Since the coordinates of the vector of state variables are autonomous we have that the action of the generator commutes with that of the differential operator $\partial^{2} / \partial x_{k} \partial x_{l}$ for $k \neq l$. Using this property in conjunction with an argument similar to that which led to equation (88) we obtain that

$$
\lim _{t \rightarrow T} \frac{\partial^{4} H_{i}(t, x)}{\partial x_{j}^{2} \partial x_{k} \partial x_{l}}=0
$$

for all $k \neq l$ and the validity of equation (92) now follows from equation (91) and the result of Lemma D.7.

In order to complete the proof it remains to show that the derivatives

$$
\frac{\partial Q_{i}}{\partial t}, \quad \frac{\partial^{2} Q_{i}}{\partial t^{2}}, \quad \frac{\partial^{2} Q_{i}}{\partial t \partial x_{j}}, \quad \frac{\partial^{3} Q_{i}}{\partial t^{3}}, \quad \frac{\partial^{3} Q_{i}}{\partial t^{2} \partial x_{j}} \quad \text { and } \frac{\partial^{3} Q_{i}}{\partial t \partial x_{j} \partial x_{k}}
$$

are all continuous at the terminal time. The continuity of $\partial Q_{i} / \partial t$ follows from equation (83), Assumption A and the fact that $Q_{i} \in C^{0,3}((0, T] \times \mathcal{X})$ by application of the first part of the proof. On the other hand differentiating both sides of equation (83) with respect to the space variables gives

$$
\frac{-\partial^{k+1} Q_{i}}{\partial t \cdot \partial x_{1}^{k_{1}} \cdots \partial x_{d}^{k_{d}}}(t, x)=\frac{\partial^{k}\left(m g_{i}+\mathcal{L} Q_{i}\right)}{\partial x_{1}^{k_{1}} \cdots \partial x_{d}^{k_{d}}}(t, x)
$$

for all $k \in \mathbb{N}$ and it follows that $Q_{i} \in C^{1,3}((0, T] \times \mathcal{X})$ since we know from Assumption A, Proposition 2 and the first part of the proof that the right hand side is continuous at the terminal time. Similarly, differentiating both sides of equation (83) with respect to time we obtain

$$
-\frac{\partial^{2} Q_{i}}{\partial t^{2}}(t, x)=\frac{\partial\left(m g_{i}\right)}{\partial t}(t, x)+\sum_{j=1}^{d} \frac{1}{2 a_{j}\left(x_{j}\right)^{2}} \frac{\partial^{3} Q_{i}}{\partial t \partial x_{j}^{2}}(t, x)
$$

and the continuity of the second order time derivative follows from Assumption A, 
Proposition 2 and the fact that $Q_{i} \in C^{1,3}((0, T] \times \mathcal{X})$. The continuity of the third order time derivative can be established similarly by differentiating the above expression first with respect to $x$ and then with respect to time. We omit the details.

QED.

Proof of Theorem 6. Combining Lemma D.6, Lemma D.9, Theorem A.1 and Theorem A.2 we obtain that the candidate price function is real analytic in $(t, x) \in[0, T] \times \mathcal{X}$ and belongs to $C^{3}((0, T] \times \mathcal{X})$. The desired result now follows from Theorem 2 and Proposition 4.

QED.

ProOF OF LEMma 1. Using well-known results on square root diffusion processes, see for example Feller (1951) and Cox et al. (1985), we have that the transition density of the $i$-th coordinate is explicitly given by

$$
q_{i}(t, x, y)=\frac{1}{\tau_{i}(t)}\left(\frac{y}{e^{-b_{i} t} x}\right)^{\frac{\nu_{i}}{2}} e^{-\frac{y+e^{-b_{i} t_{x}}}{\tau_{i}(t)}} I_{\nu_{i}}\left(\frac{2}{\tau_{i}(t)} \sqrt{e^{-b_{i} t} x y}\right)
$$

for all $(t, x, y) \in[0, T] \times(0, \infty)^{2}$ where the function $I_{\nu_{i}}$ is the modified Bessel function of the first kind with index $\nu_{i}$ and we have set

$$
\tau_{i}(t) \equiv \frac{\sigma_{i}^{2}}{2 b_{i}}\left(1-e^{-b_{i} t}\right)
$$

Since the function $\tau_{i}$ is uniformly bounded above and away from zero on $[0, T]$ we have that there are constants $C_{i}=C_{i}(T)>0$ such that

$$
C_{1} t \leq \tau_{i}(t) \leq C_{2} t
$$

Combining this property with Lemma D.10 below and the fact that $I_{\nu_{i}}(x) \leq K_{0}(x / 2)^{\nu_{i}} e^{x}$ for some strictly positive constant $K_{0}$ (see for example Joshi and Bissu (1991)) we obtain that the transition density satisfies

$$
\begin{aligned}
\left|q_{i}(t, x, y)\right| & \leq K_{0} \tau_{i}(t)^{-\left(1+\nu_{i}\right)} y^{\nu_{i}} e^{-\frac{1}{\tau_{i}(t)}\left(\sqrt{y}-\sqrt{e^{-b_{i} t} x}\right)^{2}} \\
& \leq K_{1} t^{-\left(1+\nu_{i}\right)} y^{\nu_{i}} e^{-\frac{1}{\tau_{i}(t)}\left(\sqrt{y}-\sqrt{e^{-b_{i} t} x}\right)^{2}} \\
& \leq K_{2} t^{-\left(1+\nu_{i}\right)} y^{\nu_{i}} e^{K_{3}(x-y)-\frac{K_{4}}{t}(\sqrt{y}-\sqrt{x})^{2}}
\end{aligned}
$$

for some strictly positive constants $\left(K_{0}\right)_{i=1}^{4}$ and the validity of Assumption G now follows from the definition of the function $M_{i}$.

In order to complete the proof assume that the function $f$ satisfies (36). Combining this assumption with the elementary inequalities

$$
\|x\|^{\delta} \leq\left(\sum_{i=1}^{d}\left|x_{i}\right|\right)^{\delta} \leq \sum_{i=1}^{d} d^{\delta}\left|x_{i}\right|
$$

we deduce that

$$
\int_{\mathcal{X}} \hat{f}(y) \prod_{i=1}^{d} L_{i}\left(y_{i}\right) e^{-\varepsilon M_{i}\left(y_{i}\right)^{2}} d y \leq K \prod_{i=1}^{d} \int_{\mathcal{X}_{i}} y_{i}^{\nu_{i}} e^{\phi\left|y_{i}\right|^{\delta}-\varepsilon M_{i}\left(y_{i}\right)^{2}} d y_{i}
$$

for some strictly positive constant $\phi$ and the desired result now follows from the definition of the function $M_{i}$ and the fact that $\delta<1$.

QED. 
LEMMA D.10: We have

$$
e^{-\frac{\left(y-e^{-t} x\right)^{2}}{1-e^{-2 t}}} \leq e^{-\frac{1}{2 t}(y-x)^{2}+\frac{t}{2}+\frac{x^{2}-y^{2}}{2}}
$$

for all $(t, x, y) \in \mathbb{R}_{+} \times \mathbb{R}^{2}$.

Proof. See Downes (2009, Section 3.1).

QED.

Proof of Proposition 7. The result follows from Lemma 1 and Proposition 10 after setting $\xi(x)=\|x\|^{\delta}$ for some $\delta \in(0,1)$.

QED.

Proof of Lemma 2. The result follows from equation (35) and Lemma 1. We omit the details.

QED.

Proof of Proposition 8. The result follows from Lemma 2 and Proposition 10 after setting $\xi(x)=A+B \log \left(1+K\|x\|^{\alpha}\right)$ for some suitably chosen constants $A, B, K$ and $\alpha<\bar{\alpha}$. We omit the details.

QED.

\section{D.3 Counterexamples}

Proof of Proposition 9. Assume without loss of generality that $d=2$ and consider the nonnegative process defined by

$$
H_{t}=H\left(t, X_{t}\right) \equiv E_{t}\left[e^{-\rho T+\int_{t}^{T} \Phi_{s} d s} v^{\prime}\left(\bar{G}\left(X_{T}\right)\right) G_{1}\left(X_{T}\right)\right]
$$

with

$$
\Phi_{t}=\Phi\left(t, X_{t}\right) \equiv \frac{g_{1}(t, x)}{K+F_{1}(t, x)} .
$$

Since all the terms are bounded we know that $H$ is bounded and choosing $K$ large enough it can be guaranteed that the function

$$
g_{2}(t, x) \equiv\left(u^{\prime}\right)^{-1}\left(\frac{e^{\rho t} H(t, x)}{K+F_{1}(t, x)}\right)-g_{1}(t, x)
$$

is nonnegative for all $(t, x)$. Taking $g_{2}$ as the intermediate dividend on the second stock we obtain that the equilibrium consumption price process is

$$
e^{\rho t} m\left(t, X_{t}\right)=1_{\{t<T\}} u^{\prime}\left(\bar{g}\left(t, X_{t}\right)\right)+1_{\{t=T\}} v^{\prime}\left(\bar{G}\left(X_{t}\right)\right)
$$

and it follows that the (pre-horizon) equilibrium price of the first stock is

$$
\begin{aligned}
& m\left(t, X_{t}\right) P_{1}\left(t, X_{t}\right)=\frac{H_{t} P_{1}\left(t, X_{t}\right)}{F_{1}\left(t, X_{t}\right)+K} \\
& =E_{t}\left[\int_{t}^{T} m\left(\tau, X_{\tau}\right) g_{1}\left(\tau, X_{\tau}\right) d \tau+e^{-\rho T} m\left(T, X_{T}\right) G_{1}\left(X_{T}\right)\right]
\end{aligned}
$$

Using this expression in conjunction with the definition of the functions $g_{2}$ and $m$ we easily get that

$$
d\left(\frac{H_{t} P_{1}\left(t, X_{t}\right)}{K+F_{1}\left(t, X_{t}\right)}\right)=-\Phi_{t} H_{t} d t+d M_{t}^{P}
$$


for some uniformly integrable martingale $M^{P}$. On the other hand, we have that the dynamics of the process $H$ are given by

$$
d H_{t}=-\Phi_{t} H_{t} d t+e^{-\int_{0}^{t} \Phi\left(s, X_{s}\right) d s} d M_{t}=-\Phi_{t} H_{t} d t+d M_{t}^{H}
$$

where $M$ is the bounded martingale defined by

$$
\begin{aligned}
M_{t} & \equiv e^{\int_{0}^{t} \Phi_{s} d s} H_{t}-H_{0} \\
& =E_{t}\left[e^{-\rho T+\int_{0}^{T} \Phi_{s} d s} v^{\prime}\left(\bar{G}\left(X_{T}\right)\right) G_{1}\left(X_{T}\right)\right]-H_{0} .
\end{aligned}
$$

Using the boundedness of $M$ in conjunction with the nonnegativity of $\Phi$ and the Burkholder-Davis-Gundy inequalities (see Karatzas and Shreve (1998, Theorem 3.28)) we get that

$$
E_{0}\left[\sup _{t \in[0, T]}\left|M_{t}^{H}\right|\right] \leq C \cdot E_{0}\left[\sup _{t \in[0, T]}\left|M_{t}\right|\right]<\infty
$$

for some strictly positive constant $C$ and it follows that $M^{H}$ is a uniformly integrable martingale. Using this property in conjunction with equation (93) we deduce that

$$
N_{t}=H_{t}\left(1-\frac{P_{1}\left(t, X_{t}\right)}{K+F_{1}\left(t, X_{t}\right)}\right)=N_{0}+M_{t}^{H}-M_{t}^{P}
$$

is also a uniformly integrable martingale and since $\lim _{t \rightarrow T} N_{t}=0$ by definition of $g_{2}$ and $H$ we finally conclude that $P_{1}=K+F_{1}$.

QED. 